JOURNAL OF THE

AMERICAN MATHEMATICAL SOCIETY

Volume 15, Number 1, Pages 113-165

S 0894-0347(01)00382-4

Article electronically published on September 19, 2001

\title{
ANNIHILATION THEOREM AND SEPARATION THEOREM FOR BASIC CLASSICAL LIE SUPERALGEBRAS
}

\author{
MARIA GORELIK
}

\section{INTRODUCTION}

1.1. Let $\mathfrak{g}$ be a complex semisimple Lie algebra, let $\mathcal{U}$ be its universal enveloping algebra, and let $\mathcal{Z}(\mathfrak{g})$ be the centre of $\mathcal{U}$. Consider $\mathcal{U}$ as a $\mathfrak{g}$-module with respect to the adjoint action. The Separation Theorem of Kostant (see $[\mathrm{Ko}$ ) states the existence of a submodule $H$ of $\mathcal{U}$ such that the multiplication map provides the bijection $H \otimes \mathcal{Z}(\mathfrak{g}) \stackrel{\sim}{\longrightarrow} \mathcal{U}$. Moreover the multiplicity of each simple finite-dimensional module $V$ in $H$ is equal to the dimension of its zero weight space. Such an ad $\mathfrak{g}$ invariant subspace $H$ is called a harmonic space. An easy proof of the Separation Theorem was found by Bernstein and Lunts - see [BL]. This theorem is an important ingredient in the proof of the annihilation theorem of Duflo (see $[\mathrm{D}], 8.4 .3$ ) asserting that the annihilator of a Verma module is generated by its intersection with $\mathcal{Z}(\mathfrak{g})$. The annihilation theorem is reproven by Joseph and G. Letzter. They also generalize it to the quantum case - see [JL], JJ3].

In this paper we obtain analogous theorems in the case of basic classical Lie superalgebras. This was done earlier for the completely reducible case - see [M1], GL1.

Let $\mathfrak{g}=\mathfrak{g}_{0} \oplus \mathfrak{g}_{1}$ be a basic classical Lie superalgebra, let $\widetilde{\mathcal{U}}$ be its universal enveloping superalgebra, and let $\mathcal{Z}(\mathfrak{g})$ be the centre of $\widetilde{\mathcal{U}}$. Let $T$ be a special ghost element constructed in G1 - see 3.3. This element acts on the even component of a Verma module by multiplication by a scalar and on the odd component by multiplication by the opposite scalar. Call a Verma $\mathfrak{g}$-module strongly typical if it is not annihilated by $T$. A strongly typical Verma module is typical. For $\mathfrak{g} \neq$ $B(m, n), G(3)$ a Verma module is typical iff it is strongly typical.

We prove the following version of the Annihilation Theorem.

1.1.1. Theorem. The annihilator of a strongly typical Verma module $\widetilde{M}$ is a centrally generated ideal.

Moreover, for a strongly typical Verma module $\widetilde{M}$, we describe the quotient $\widetilde{\mathcal{U}} /$ Ann $\widetilde{M}$ as an ad $\mathfrak{g}$-module and show that the natural map from $\widetilde{\mathcal{U}} /$ Ann $\widetilde{M}$ to the locally finite part of $\operatorname{End}_{\mathbb{C}}(\widetilde{M})$ is bijective.

Received by the editors December 6, 2000

2000 Mathematics Subject Classification. Primary 17B10, 17B20, 17B35.

Key words and phrases. Basic classical Lie superalgebra, adjoint action, Verma module.

The author was partially supported by TMR Grant No. FMRX-CT97-0100. Research at MSRI was supported in part by NSF grant DMS-9701755. 
The converse of Theorem 1.1.1 is proven for $\mathfrak{g}$ of type I: if the annihilator of a Verma module $\widetilde{M}$ is a centrally generated ideal, then $\widetilde{M}$ is typical.

1.1.2. The proof of Theorem 1.1.1 goes as follows. As in [JL], we use the Parthasarathy-Ranga-Rao-Varadarajan (PRV) determinants. We generalize the notion of PRV determinants (see $\underline{\text { PRV] }}$ ) to basic classical Lie superalgebras. Our construction is based on the fact that the two-sided ideal $\widetilde{\mathcal{U}} T=T \widetilde{\mathcal{U}}$, considered as an ad $\mathfrak{g}$-module, is injective in a category of locally finite modules.

In the completely reducible case one assigns to each simple finite-dimensional module $\widetilde{V}$ a PRV determinant which is a polynomial in $\mathcal{S}(\mathfrak{h})$.

On the contrary, for the non-completely reducible case the lack of harmonic space forces us to substitute the PRV determinant by a set of PRV determinants corresponding to the same $\widetilde{V}$. However, we do not have to calculate these determinants, but only verify that they are non-zero. We use these determinants to show that if the locally finite part $F(\widetilde{M}, \widetilde{M})$ of the endomorphisms $\operatorname{End}_{\mathbb{C}}(\widetilde{M})$ of a strongly typical Verma module $\widetilde{M}$ has "a right size" as an ad $\mathfrak{g}$-module and the natural map $\widetilde{\mathcal{U}} \rightarrow F(\widetilde{M}, \widetilde{M})$ is surjective, then Ann $\widetilde{M}$ is centrally generated.

For type I we directly verify both conditions. The crucial point in the study of the type II case is the construction in Section 8 of a perfect mate $\chi \in \operatorname{Max} \mathcal{Z}\left(\mathfrak{g}_{0}\right)$ for each strongly typical $\tilde{\chi} \in \operatorname{Max} \mathcal{Z}(\mathfrak{g})$. We call a maximal ideal $\widetilde{\chi} \in \operatorname{Max} \mathcal{Z}(\mathfrak{g})$ strongly typical if it does not contain $T^{2}$ where $T$ is the ghost element mentioned above. We call a maximal ideal $\chi \in \operatorname{Max} \mathcal{Z}\left(\mathfrak{g}_{0}\right)$ a perfect mate for $\tilde{\chi} \in \operatorname{Max} \mathcal{Z}(\mathfrak{g})$ if the following conditions are satisfied:

(i) For any Verma $\mathfrak{g}$-module annihilated by $\widetilde{\chi}$, its $\mathfrak{g}_{0}$-submodule annihilated by a power of $\chi$ is a Verma $\mathfrak{g}_{0}$-module.

(ii) Any non-trivial $\mathfrak{g}$-module annihilated by $\widetilde{\chi}$ has a non-trivial $\mathfrak{g}_{0}$-submodule annihilated by $\chi$.

Condition (ii) seems to be difficult to check. However, it turns out that it is enough to verify (ii) only for simple highest weight $\mathfrak{g}$-modules. This is deduced from [M2].

For $\mathfrak{g}=\mathfrak{o} \mathfrak{s p}(1,2 l)$ the annihilator of a Verma $\mathfrak{g}$-module $\widetilde{M}$ is a centrally generated ideal iff $\widetilde{M}$ is strongly typical - see [GL1. In this paper we prove the similar equivalence for the basic classical Lie superalgebras of type I.

As shown in [PS1, if $\mathfrak{g}$ has type I, then for any strongly typical $\tilde{\chi} \in \mathcal{Z}(\mathfrak{g})$ the algebra $\widetilde{\mathcal{U}} /(\tilde{\mathcal{U}} \widetilde{\chi})$ is the matrix algebra over $\mathcal{U}\left(\mathfrak{g}_{0}\right) /\left(\mathcal{U}\left(\mathfrak{g}_{0}\right) \chi\right)$ for a suitable $\chi \in$ $\operatorname{Max} \mathcal{Z}\left(\mathfrak{g}_{0}\right)$. As pointed out by V. Serganova this result implies Theorem 1.1.1 for the type I case. The opposite implication is also easy (see 7.4).

1.2. Let $\mathfrak{g}$ be a basic classical Lie superalgebra which is not completely reducible. Then $\widetilde{\mathcal{U}}$ is not a domain (see [AL]) and $\mathcal{Z}(\mathfrak{g})$ is not Noetherian (피, 2.8). However all non-zero central elements are non-zero divisors and $\mathcal{Z}(\mathfrak{g})$ contains an element $z$ such that the localized algebra $\mathcal{Z}(\mathfrak{g})\left[z^{-1}\right]$ is isomorphic to a localization of a polynomial algebra. One can take $z:=T^{2}$ where $T$ is the element mentioned above. The element $T$ is even; it commutes with the even elements of $\widetilde{\mathcal{U}}$ and anticommutes with the odd ones. Moreover, the image of $T$ in the symmetric algebra $\mathcal{S}(\mathfrak{g})$ belongs to the top exterior power of $\mathfrak{g}_{1}$. These properties determine $T$ up to a scalar. 
It is easy to show (see G1], 4.5) that $\widetilde{\mathcal{U}}$, considered as an ad $\mathfrak{g}$-module, does not admit a factorization of the form $H \otimes \mathcal{Z}(\mathfrak{g})$. For type I Lie superalgebras, we prove the following version of the Separation Theorem.

1.2.1. Theorem. For $\mathfrak{g}$ of type $I$, there exists an ad $\mathfrak{g}$-invariant subspace $H$ of $\widetilde{\mathcal{U}}$ such that the multiplication map provides the bijection $H \otimes \mathcal{Z}(\mathfrak{g})\left[T^{-2}\right] \rightarrow \widetilde{\mathcal{U}}\left[T^{-2}\right]$.

Clearly, $\mathcal{Z}(\mathfrak{g})\left[T^{-2}\right]$ coincides with the centre of $\widetilde{\mathcal{U}}\left[T^{-2}\right]$. As an ad $\mathfrak{g}$-module, $H$ is injective in an appropriate category of locally finite modules and for any simple finite-dimensional module $\widetilde{V}$ one has $\operatorname{dim} \operatorname{Hom}_{\mathfrak{g}}(\widetilde{V}, H)=\left.\operatorname{dim} \widetilde{V}\right|_{0}$ where $\left.\widetilde{V}\right|_{0}$ is the zero weight space of $\widetilde{V}$. For a basic classical Lie superalgebra of type II, we obtain a weaker result, namely that the similar assertions hold if we substitute $z$ by a certain subset $S$ of $\mathcal{Z}(\mathfrak{g})$. This set $S$ can be described in terms of the PRV determinants.

A natural conjecture is that one can always choose $S$ equal to $\left\{T^{2}\right\}$. A possible way to prove this conjecture is to show that an irreducible factor of a PRV determinant is either a factor of Shapovalov form or is of the form $\left(\beta^{\vee}+\beta^{\vee}(\rho)\right)$ for some odd coroot $\beta$. However it is not clear how to calculate these determinants if $\mathfrak{g}$ is not completely reducible.

1.3. Content of the paper. In Section 2 we recall some facts about the basic classical Lie superalgebras.

In Section 3 we define a category $\mathcal{F}$ in of locally finite $\mathfrak{g}$-modules and provide some properties of $\mathcal{F}$ in. We also recall the construction and properties of the element $T$.

In Section 4 we investigate the $\mathfrak{g}$-module structure of $\widetilde{\mathcal{U}}$ given by the adjoint action. We start with studying $\operatorname{Hom}_{\mathfrak{g}}(\widetilde{V}, \widetilde{\mathcal{U}})$ for a simple finite-dimensional module $\widetilde{V}$. For each $\widetilde{V}$ we construct a central element $z$ such that the localized module $\operatorname{Hom}_{\mathfrak{g}}\left(\widetilde{V}, \widetilde{\mathcal{U}}\left[z^{-1}\right]\right)=\operatorname{Hom}_{\mathfrak{g}}(\widetilde{V}, \widetilde{\mathcal{U}})\left[z^{-1}\right]$ is a free $\mathcal{Z}(\mathfrak{g})\left[z^{-1}\right]$-module whose rank is equal to $\left.\operatorname{dim} \widetilde{V}\right|_{0}$. In 4.6 we generalize a notion of PRV determinants to the case of non-completely reducible Lie superalgebras. We also establish properties of these determinants which are similar to the properties of the original PRV determinants. In 4.7 we show that for a suitable subset $S$ of $\mathcal{Z}(\mathfrak{g})$ the localized algebra $\widetilde{\mathcal{U}}\left[S^{-1}\right]$ is free over its centre $\mathcal{Z}(\mathfrak{g})\left[S^{-1}\right]$. We describe the ad $\mathfrak{g}$-module structure of the corresponding "generic harmonic space" $H$. We show that one may choose $H$ to be the ad $\mathfrak{g}$-module generated by $H^{\prime} T$ where $H^{\prime}$ is a certain harmonic space of $\mathcal{U}\left(\mathfrak{g}_{0}\right)$.

In Section 5 we establish a connection between PRV determinants and the annihilators of simple modules. We show that if all PRV determinants do not vanish at a point $\lambda \in \mathfrak{h}^{*}$ and if a simple module $\widetilde{V}(\lambda)$ is strongly typical, then its annihilator is a centrally generated ideal. Moreover, for a simple strongly typical Verma module $\widetilde{V}(\lambda)$ all PRV determinants do not vanish at a point $\lambda \in \mathfrak{h}^{*}$ iff the natural map $\widetilde{\mathcal{U}} \rightarrow F(\widetilde{M}, \widetilde{M})$ is surjective and $F(\widetilde{M}, \widetilde{M})$ has a certain nice structure as an ad $\mathfrak{g}$-module (it should be isomorphic to the "generic harmonic space" $H$ ).

Sections 6 and 7 are devoted to the type I case. Preliminary facts are concentrated in Section [6] In Section 7 we prove that for a suitable ad $\mathfrak{g}$-stable $H$ the multiplication map provides an isomorphism $H \otimes \mathcal{Z}(\mathfrak{g})\left[T^{-2}\right] \rightarrow \widetilde{\mathcal{U}}\left[T^{-2}\right]$. We also prove that the annihilator of a Verma module is centrally generated iff this module is strongly typical.

Sections 8 and 9 are devoted to the type II case.

In Section 8 we describe for each strongly typical $\tilde{\chi} \in \operatorname{Max} \mathcal{Z}(\mathfrak{g})$ its perfect mate $\chi \in \operatorname{Max} \mathcal{Z}\left(\mathfrak{g}_{0}\right)$. Note that in the type I case for any strongly typical $\tilde{\chi} \in \operatorname{Max} \mathcal{Z}(\mathfrak{g})$ 
and for any $\lambda$ such that $\widetilde{\chi}$ annihilates $\widetilde{M}(\lambda)$, the ideal $\operatorname{Ann}_{\mathcal{Z}\left(\mathfrak{g}_{0}\right)} M(\lambda)$ is a perfect mate for $\widetilde{\chi}$. This does not hold for type II. For certain "generic" $\tilde{\chi}$, the ideal $\operatorname{Ann}_{\mathcal{Z}\left(\mathfrak{g}_{0}\right)} M(\lambda)$ is a perfect mate if one chooses $\lambda$ satisfying, apart from $\widetilde{\chi} \widetilde{M}(\lambda)=0$, also a kind of "dominance" condition. For $B(m, n)$ and $G(3)$ all strongly typical central characters are generic. For the remaining superalgebras $D(m, n), D(2,1, \alpha)$ and $F(4)$ we select perfect mates for non-generic strongly typical central characters case by case.

In Section 9 we prove Theorem 1.1.1. The existence of a perfect mate for each strongly typical $\tilde{\chi} \in \operatorname{Max} \mathcal{Z}(\mathfrak{g})$ enables us to show that for a Verma module $\widetilde{M}$ with the central character $\tilde{\chi}$ the natural map $\widetilde{\mathcal{U}} \rightarrow F(\widetilde{M}, \widetilde{M})$ is surjective and $F(\widetilde{M}, \widetilde{M}) \cong H$. According to Section 5 these two conditions imply that the annihilator of $\widetilde{M}$ is a centrally generated ideal.

In Section 10 we study the $\mathfrak{g}_{0}$-structure of Verma $\mathfrak{g}$-modules.

Appendix 11 contains some lemmas used in the main text.

\section{Preliminaries}

In this paper the ground field is $\mathbb{C}$. Everywhere in the paper, apart from Sections [6] and [7, $\mathfrak{g}=\mathfrak{g}_{0} \oplus \mathfrak{g}_{1}$ denotes one (unless otherwise specified, an arbitrary one) of the basic classical complex Lie superalgebras $\mathfrak{g l}(m, n), \mathfrak{s l}(m, n), \mathfrak{o s p}(m, n)$, $\mathfrak{p s l}(n, n)$. Each of these Lie superalgebras possesses the following properties: it admits a $\mathfrak{g}$-invariant bilinear form which is non-degenerate on $[\mathfrak{g}, \mathfrak{g}]$ and the even part $\mathfrak{g}_{0}$ is a reductive Lie algebra.

In Sections [6 and [7 $\mathfrak{g}=\mathfrak{g}_{0} \oplus \mathfrak{g}_{\mathrm{T}}$ denotes one of the basic classical complex Lie superalgebras of type I: $\mathfrak{g l}(m, n), \mathfrak{s l}(m, n), \mathfrak{o s p}(2, n), \mathfrak{p s l}(n, n)$. We shall slightly change our notation since these superalgebras admit $\mathbb{Z}$-grading $\mathfrak{g}=\mathfrak{g}_{-1} \oplus \mathfrak{g}_{0} \oplus \mathfrak{g}_{1}$. The even part $\mathfrak{g}_{\overline{0}}$ coincides with $\mathfrak{g}_{0}$ and the odd part $\mathfrak{g}_{\overline{1}}$ is the sum of two dual $\mathfrak{g}_{0}$-modules $\mathfrak{g}_{-1}$ and $\mathfrak{g}_{1}$.

In this section we present the main preliminary facts about the structure of the basic classical complex Lie superalgebras and their representations, which we shall use in this paper.

2.1. Conventions. We denote by $\mathbb{N}^{+}$the set of positive integers and by $\# I$ the number of elements of the given set $I$. If $A$ is an algebra, $N$ is an $A$-module and $X, Y$ are subsets of $A$ and $N$, respectively, then we denote by $X Y$ the submodule spanned by the products $x y$ where $x \in X, y \in Y$.

For a $\mathbb{Z}_{2}$-homogeneous element $u$ of a superalgebra denote by $d(u)$ its $\mathbb{Z}_{2}$-degree. In all formulas where this notation is used, $u$ is assumed to be $\mathbb{Z}_{2}$-homogeneous.

For a superalgebra $\mathfrak{m}$ denote by $\mathcal{U}(\mathfrak{m})$ its universal enveloping superalgebra. All modules in this text are assumed to be left modules unless otherwise specified. An $\mathfrak{m}$-module $N$ is called locally finite if $\operatorname{dim} \mathcal{U}(\mathfrak{m}) v<\infty$ for all $v \in N$. For a given module $N$ we denote by $N^{\oplus r}$ the direct sum of $n$-copies of $N$. Set $\widetilde{\mathcal{U}}:=\mathcal{U}(\mathfrak{g})$ and $\mathcal{U}:=\mathcal{U}\left(\mathfrak{g}_{0}\right)$.

The symbol $\widetilde{V}$ (resp., $V$ ) is always used for a simple $\mathfrak{g}$ - (resp., $\mathfrak{g}_{0^{-}}$)module and the symbol $\widetilde{M}$ (resp., $M$ ) for a Verma $\mathfrak{g}$ - (resp., $\mathfrak{g}_{0^{-}}$)module.

2.2. Triangular decompositions. Triangular decompositions of the superalgebras are defined in [PS2 as follows. A Lie subsuperalgebra $\mathfrak{h} \subset \mathfrak{g}$ is called a Cartan subsuperalgebra if $\mathfrak{h}$ is nilpotent and coincides with its centralizer in $\mathfrak{g}$. For the basic 
classical Lie superalgebras the set of Cartan subalgebras coincides with the set of Cartan subalgebras of $\mathfrak{g}_{0}$. Fix a Cartan subalgebra $\mathfrak{h}$; it acts semisimply on $\mathfrak{g}$ :

$$
\mathfrak{g}:=\left.\bigoplus_{\mu \in \mathfrak{h}^{*}} \mathfrak{g}\right|_{\mu},\left.\quad \mathfrak{g}\right|_{\mu}:=\{a \in \mathfrak{g} \mid \forall h \in \mathfrak{h},[h, a]=\mu(a)\}
$$

Denote by $\Delta$ the set of non-zero roots that is the set $\left\{\alpha \in \mathfrak{h}^{*}|\mathfrak{g}|_{\alpha} \neq 0\right\} \backslash\{0\}$. An element $h \in \mathfrak{h}$ is called regular if $\operatorname{Re} \alpha(h) \neq 0$ for all $\alpha \in \Delta$. Any regular element determines the decomposition $\Delta=\Delta^{+} \amalg \Delta^{-}$where

$$
\Delta^{+}:=\{\alpha \in \Delta \mid \operatorname{Re} \alpha(h)>0\}, \quad \Delta^{-}:=\{\alpha \in \Delta \mid \operatorname{Re} \alpha(h)<0\} .
$$

Moreover it determines a decomposition $\mathfrak{g}=\mathfrak{n}^{-} \oplus \mathfrak{h} \oplus \mathfrak{n}^{+}$where

$$
\mathfrak{n}^{+}:=\left.\bigoplus_{\alpha \in \Delta^{+}} \mathfrak{g}\right|_{\alpha}, \quad \mathfrak{n}^{-}:=\left.\bigoplus_{\alpha \in \Delta^{-}} \mathfrak{g}\right|_{\alpha}
$$

Such decompositions of $\mathfrak{g}$ are called triangular decompositions. It is clear that $\mathfrak{n}^{ \pm}$ are nilpotent Lie subsuperalgebras of $\mathfrak{g}$.

A Lie subsuperalgebra $\mathfrak{b} \subset \mathfrak{g}$ is called a Borel subsuperalgebra if $\mathfrak{b}=\mathfrak{h} \oplus \mathfrak{n}^{+}$ for some triangular decomposition $\mathfrak{g}=\mathfrak{n}^{-} \oplus \mathfrak{h} \oplus \mathfrak{n}^{+}$. A Borel subsuperalgebra determines the triangular decomposition; we will add a lower index to designate the corresponding Borel subsuperalgebra in the case where the choice of triangular decomposition is not clear from the context. We denote by $\Delta(\mathfrak{b})$ the set of non-zero roots of $\mathfrak{b}$. We say that a vector $v$ of a $\mathfrak{g}$-module is $\mathfrak{b}$-primitive if $[\mathfrak{b}, \mathfrak{b}] v=0$ and $\mathfrak{h} v \in \mathbb{C} v$.

A triangular decomposition $\mathfrak{g}=\mathfrak{n}^{-} \oplus \mathfrak{h} \oplus \mathfrak{n}^{+}$induces the triangular decomposition of the even part $\mathfrak{g}_{0}=\mathfrak{n}_{0}^{-} \oplus \mathfrak{h} \oplus \mathfrak{n}_{0}^{+}$. The group of inner automorphisms of $\mathfrak{g}_{0}$ acts transitively on all triangular decompositions of $\mathfrak{g}_{0}$ and the action of this group can be extended to $\mathfrak{g}$. Hence the theory does not depend on the choice of a triangular decomposition of $\mathfrak{g}_{0}$. In the sequel we fix a triangular decomposition $\mathfrak{g}_{0}=\mathfrak{n}_{0}^{-} \oplus \mathfrak{h} \oplus$ $\mathfrak{n}_{0}^{+}$and consider triangular decompositions of $\mathfrak{g}$ which induce this fixed triangular decomposition of $\mathfrak{g}_{0}$.

2.2.1. Denote by $\Delta_{0}$ the set of non-zero even roots of $\mathfrak{g}$ and by $\Delta_{1}$ the set of odd roots of $\mathfrak{g}$. Set $\Delta_{0}^{ \pm}:=\Delta_{0} \cap \Delta^{ \pm}$and $\Delta_{1}^{ \pm}:=\Delta_{1} \cap \Delta^{ \pm}$.

Denote by $(-,-)$ a $\mathfrak{g}$-invariant bilinear form on $\mathfrak{g}$ which is non-degenerate on $[\mathfrak{g}, \mathfrak{g}]$ and the induced $W$-invariant bilinear form on $\mathfrak{h}^{*}$. A root $\alpha \in \Delta$ is called isotropic if $(\alpha, \alpha)=0$. For a root $\alpha$ denote by $\alpha^{\vee}$ the element of $\mathfrak{h}$ satisfying $\alpha^{\vee}(\mu)=(\alpha, \mu)$ for each $\mu \in \mathfrak{h}^{*}$.

Set

$$
\bar{\Delta}_{0}^{+}:=\left\{\alpha \in \Delta_{0}^{+} \mid \alpha / 2 \notin \Delta_{1}^{+}\right\}, \quad \bar{\Delta}_{1}^{+}:=\left\{\beta \in \Delta_{1}^{+} \mid 2 \beta \notin \Delta_{0}^{+}\right\} .
$$

The set of positive isotropic roots coincides with $\bar{\Delta}_{1}^{+}$.

Remark that $\sum_{\alpha \in \Delta^{+}} n_{\alpha} \alpha=0$ for some $n_{\alpha} \in \mathbb{N}$ implies $n_{\alpha}=0$ for all $\alpha \in \Delta^{+}$. This allows us to define the standard partial order relation on $\mathfrak{h}^{*}$ by $\lambda \leq \mu \Longleftrightarrow$ $\mu-\lambda \in \sum_{\alpha \in \Delta^{+}} \mathbb{N} \alpha$. One can easily sees that the minimal (with respect to this partial order) elements of $\Delta^{+}$form a basis of simple roots.

Denote by $\pi_{0}$ the basis of simple roots of $\mathfrak{g}_{0}$ and by $W$ the Weyl group of $\Delta_{0}$. Denote by $|W|$ the number of elements in $W$. For $w \in W$ set $\operatorname{sn}(w):=(-1)^{l(w)}$ where $l(w)$ is the length of $w$. For a non-isotropic root $\alpha$ define $s_{\alpha} \in$ Aut $\mathfrak{h}^{*}$ by 
setting

$$
s_{\alpha}(\lambda):=\lambda-2 \frac{(\alpha, \lambda)}{(\alpha, \alpha)} \alpha .
$$

Evidently $s_{k \alpha}=s_{\alpha}$ and so the subgroup of Aut $\mathfrak{h}^{*}$ generated by the $s_{\alpha}$ coincides with $W$.

Set

$$
\rho_{0}:=\frac{1}{2} \sum_{\alpha \in \Delta_{0}^{+}} \alpha, \quad \rho_{1}:=\frac{1}{2} \sum_{\alpha \in \Delta_{1}^{+}} \alpha, \quad \rho:=\rho_{0}-\rho_{1} .
$$

For a simple root $\alpha$ one has $2(\alpha, \rho)=(\alpha, \alpha)$.

Define the translated action of $W$ on $\mathfrak{h}^{*}$ and on the symmetric algebra $\mathcal{S}(\mathfrak{h})$ by the formulas:

$$
w \cdot \lambda:=w(\lambda+\rho)-\rho, \quad w \cdot f(\lambda):=f\left(w^{-1} \cdot \lambda\right), \quad \forall \lambda \in \mathfrak{h}^{*}, w \in W .
$$

2.2.2. For a $\mathfrak{g}_{0}$-module $N$ and an element $\mu \in \mathfrak{h}^{*}$ set

$$
\left.N\right|_{\mu}:=\{m \in M \mid h m=\mu(h) m, \forall h \in \mathfrak{h}\} .
$$

We shall consider mainly $\mathfrak{h}$-diagonalizable modules, that is, satisfying $N=$ $\left.\sum_{\mu \in \mathfrak{h}^{*}} N\right|_{\mu}$. Set

$$
\Omega(N):=\left\{\mu \in \mathfrak{h}^{*}|N|_{\mu} \neq 0\right\} .
$$

If $\left.\operatorname{dim} N\right|_{\mu}<\infty$ for each $\mu \in \mathfrak{h}^{*}$, set $\operatorname{ch} N:=\sum_{\lambda \in \mathfrak{h}^{*}}\left(\left.\operatorname{dim} N\right|_{\mu}\right) e^{\mu}$.

When we use the notation $\left.\widetilde{\mathcal{U}}\right|_{\mu}$, the action of $\mathfrak{g}$ on $\widetilde{\mathcal{U}}$ is assumed to be the adjoint action.

2.3. An important property of the basic classical Lie superalgebras is the existence of a Cartan superantiautomorphism $\sigma$ coming from the supertransposition of matrices. Recall that an even linear endomorphism $\iota$ of a Lie (resp., associative) superalgebra is called a superantiautomorphism if $\iota([x, y])=(-1)^{d(x) d(y)}[\iota(y), \iota(x)]$ (resp., $\left.\iota(x y)=(-1)^{d(x) d(y)} \iota(y) \iota(x)\right)$ for all homogeneous elements $x, y$. The Cartan superantiautomorphism $\sigma$ has the following properties:

$$
\begin{array}{lll}
\text { a) } & \sigma^{2}(g)=(-1)^{d(g)} g, & \forall g \in \mathfrak{g}, \\
\text { b) } & \sigma\left(\mathfrak{n}^{+}\right)=\mathfrak{n}^{-}, & \\
\text {c) } & \sigma(h)=h, & \forall h \in \mathfrak{h} .
\end{array}
$$

The restriction of $\sigma$ to $\mathfrak{g}_{0}$ is a Cartan anti-involution:

$$
\begin{array}{ll}
\text { a) } & \left.\left(\sigma^{2}\right)\right|_{\mathfrak{g}_{0}}=\mathrm{id}, \\
\text { b) } & \sigma\left(\mathfrak{n}_{0}^{+}\right)=\mathfrak{n}_{0}^{-}, \\
\text {c) } & \sigma(h)=h, \quad \forall h \in \mathfrak{h} .
\end{array}
$$

2.3.1. Symmetric algebra. Denote by $\mathcal{F}$ the canonical filtration of $\widetilde{\mathcal{U}}$ given by $\mathcal{F}^{k}:=$ $\mathfrak{g}^{k}$. This filtration is ad $\mathfrak{g}$-invariant and the associated graded superalgebra $\mathcal{S}(\mathfrak{g})$ inherits an ad $\mathfrak{g}$-module structure. The superalgebra $\mathcal{S}(\mathfrak{g})$ is supercommutative: it is the product of the symmetric (even) algebra $\mathcal{S}\left(\mathfrak{g}_{0}\right)$ and the external superalgebra $\Lambda \mathfrak{g}_{1}$.

For $u \in \widetilde{\mathcal{U}}$ denote by gr $u$ its image in $\mathcal{S}(\mathfrak{g})$; identify $\mathcal{U}(\mathfrak{h})$ and its image $\mathcal{S}(\mathfrak{h})$. 
2.3.2. Centre. By definition, the (super)centre $\mathcal{Z}(\mathfrak{g}):=\widetilde{\mathcal{U}}^{\mathfrak{g}}$. One has gr $\mathcal{Z}(\mathfrak{g})=$ $\mathcal{S}(\mathfrak{g})^{\mathfrak{g}}$.

Denote by gr $P$ the projection $\mathcal{S}(\mathfrak{g}) \rightarrow \mathcal{S}(\mathfrak{h})$ along $\mathcal{S}(\mathfrak{g}) \operatorname{gr}\left(\mathfrak{n}^{-}+\mathfrak{n}^{+}\right)$. The restriction of gr $P$ to $\mathcal{S}(\mathfrak{g})^{\mathfrak{g}}$ provides a monomorphism $\iota: \mathcal{S}(\mathfrak{g})^{\mathfrak{g}} \rightarrow \mathcal{S}(\mathfrak{h})^{W}$. As a consequence, all non-zero elements of $\mathcal{S}(\mathfrak{g})^{\mathfrak{g}}$ (resp., $\mathcal{Z}(\mathfrak{g})$ ) are non-zero divisors in $\mathcal{S}(\mathfrak{g})$ (resp., $\widetilde{\mathcal{U}})$. The image of $\iota$ is described in [K2], [S1], [BZV]; $\iota$ is bijective iff $\mathfrak{g}=\mathfrak{o s p}(1,2 l)$.

Denote by $P_{\emptyset}$ the projection $\mathcal{S}(\mathfrak{g}) \rightarrow \mathcal{S}\left(\mathfrak{g}_{0}\right)$ with the kernel $K:=\sum_{i \geq 1} \mathcal{S}\left(\mathfrak{g}_{0}\right) \Lambda^{i} \mathfrak{g}_{1}$. It is easy to see that $K$ is ad $\mathfrak{g}_{0}$-invariant and so $P_{\emptyset}$ is an ad $\mathfrak{g}_{0}$-map. Moreover $P_{\emptyset}$ provides a monomorphism $\mathcal{S}(\mathfrak{g})^{\mathfrak{g}} \rightarrow \mathcal{S}\left(\mathfrak{g}_{0}\right)^{\mathfrak{g}_{0}}$ since gr $P=\operatorname{gr} P \circ P_{\emptyset}$ and so the injectivity of $P_{\emptyset}$ on $\mathcal{S}(\mathfrak{g})^{\mathfrak{g}}$ follows from the injectivity of gr $P$.

2.3.3. Harish-Chandra projection. Denote by $\mathcal{P}$ the Harish-Chandra projection $\widetilde{\mathcal{U}} \rightarrow \mathcal{S}(\mathfrak{h})$ with respect to the decomposition $\widetilde{\mathcal{U}}=\left(\widetilde{\mathcal{U}} \mathfrak{n}^{+}+\mathfrak{n}^{-} \widetilde{\mathcal{U}}\right) \oplus \mathcal{U}(\mathfrak{h})$. The restriction of $\mathcal{P}$ to $\left.\widetilde{\mathcal{U}}\right|_{0}=\widetilde{\mathcal{U}}^{\mathfrak{h}}$ is an algebra homomorphism. An element $a \in \widetilde{\mathcal{U}}^{\mathfrak{h}}$ acts on a primitive vector of weight $\mu$ by the multiplication by the scalar $\mathcal{P}(a)(\mu)$.

The Harish-Chandra projection provides a monomorphism $\mathcal{Z}(\mathfrak{g}) \rightarrow \mathcal{S}(\mathfrak{h})^{W}$. If $\widetilde{N}$ is a $\mathfrak{g}$-module generated by a primitive vector of weight $\lambda$, then a central element $z$ acts on $\widetilde{N}$ by the multiplication by the scalar $\mathcal{P}(z)(\lambda)$.

Call $\tilde{\chi} \in \operatorname{Max} \mathcal{Z}(\mathfrak{g})$ a central character of a $\mathfrak{g}$-module $N$ if $\tilde{\chi}^{r} N=0$ for $r>>0$.

2.3.4. Let $z$ be an element of $\mathcal{Z}(\mathfrak{g})$. Since $z$ has weight zero, $z=\mathcal{P}(z)+\sum_{i} u_{i}^{-} u_{i}^{+}$ where $u_{i}^{-} \in \mathcal{U}\left(\mathfrak{h}^{-}\right) \mathfrak{n}^{-}, u_{i}^{+} \in \mathcal{U}\left(\mathfrak{h}^{+}\right) \mathfrak{n}^{+}$for all $i$. One has $\sigma\left(u_{i}^{-} u_{i}^{+}\right)= \pm \sigma\left(u_{i}^{+}\right) \sigma\left(u_{i}^{-}\right) \in$ $\mathcal{U}\left(\mathfrak{h}^{-}\right) \mathfrak{n}^{-} \mathcal{U}\left(\mathfrak{h}^{+}\right) \mathfrak{n}^{+}$. Therefore

$$
\sigma(z)=\sigma(\mathcal{P}(z))+\sum_{i} \pm \sigma\left(u_{i}^{+}\right) \sigma\left(u_{i}^{-}\right) \in \mathcal{P}(z)+\widetilde{\mathcal{U}}^{+},
$$

that is, $\mathcal{P}(\sigma(z))=\mathcal{P}(z)$. Thus the superantiautomorphism $\sigma$ stabilizes the central elements. This implies that $\mathcal{Z}\left(\mathfrak{g}_{0}\right), \mathcal{Z}(\mathfrak{g}) \subset \tilde{\mathcal{U}}^{\sigma}$.

2.3.5. For a $\mathfrak{g}_{0}$-module $L$ denote by $\operatorname{Ind}_{\mathfrak{g}_{0}}^{\mathfrak{g}} L$ the vector space $\widetilde{\mathcal{U}} \otimes \mathfrak{U} L$ (here $\widetilde{\mathcal{U}}$ is considered as a right $\mathcal{U}$-module and a left $\widetilde{\mathcal{U}}$-module through the multiplication) equipped with the natural structure of a left $\widetilde{\mathcal{U}}$-module. Denote by $\operatorname{Coind}_{\mathfrak{g}_{0}}^{\mathfrak{g}} L$ the vector space $\operatorname{Hom}_{\mathcal{U}}(\widetilde{\mathcal{U}}, L)$ (here $\widetilde{\mathcal{U}}$ is considered as a left $\mathcal{U}$-module) equipped with the following structure of a left $\widetilde{\mathcal{U}}$-module: $(u f)\left(u^{\prime}\right):=f\left(u^{\prime} u\right)$ for any $f \in$ $\operatorname{Hom}_{\mathcal{U}}(\widetilde{\mathcal{U}}, L), u, u^{\prime} \in \widetilde{\mathcal{U}}$. For a $\mathfrak{g}$-module $\widetilde{N}$ and a $\mathfrak{g}_{0}$-module $L$ one has the canonical bijections

$$
\begin{gathered}
\operatorname{Hom}_{\mathfrak{g}_{0}}(\widetilde{N}, L) \stackrel{\sim}{\longrightarrow} \operatorname{Hom}_{\mathfrak{g}}\left(\widetilde{N}, \operatorname{Coind}_{\mathfrak{g}_{0}}^{\mathfrak{g}} L\right), \\
\operatorname{Hom}_{\mathfrak{g}_{0}}(L, \widetilde{N}) \stackrel{\sim}{\longrightarrow} \operatorname{Hom}_{\mathfrak{g}}\left(\operatorname{Ind}_{\mathfrak{g}_{0}}^{\mathfrak{g}} L, N\right) .
\end{gathered}
$$

By $[\mathrm{BF}], \operatorname{Ind}_{\mathfrak{g}_{0}}^{\mathfrak{g}} L \cong \operatorname{Coind}_{\mathfrak{g}_{0}}^{\mathfrak{g}} L$.

2.4. Hopf algebra structure. The enveloping algebra $\widetilde{\mathcal{U}}$ is a supercommutative Hopf superalgebra. This means, in particular, that the antipode $S$ is a superantiautomorphism of $\widetilde{\mathcal{U}}$ and that the comultiplication $\Delta^{\prime}: \widetilde{\mathcal{U}} \rightarrow \widetilde{\mathcal{U}} \otimes \widetilde{\mathcal{U}}$ is a homomorphism of superalgebras satisfying the relation $s \circ \Delta^{\prime}=\Delta^{\prime}$ where $s$ is a linear map 
$s: \widetilde{\mathcal{U}} \otimes \widetilde{\mathcal{U}} \rightarrow \widetilde{\mathcal{U}} \otimes \widetilde{\mathcal{U}}$ given by $s\left(u_{1} \otimes u_{2}\right):=(-1)^{d\left(u_{1}\right) d\left(u_{2}\right)}\left(u_{2} \otimes u_{1}\right)$. The Hopf algebra structure on $\tilde{\mathcal{U}}$ is given by

$$
\begin{aligned}
\Delta^{\prime}(g) & =g \otimes 1+1 \otimes g \\
\varepsilon(g) & =0 \\
S(g) & =-g
\end{aligned}
$$

for any $g \in \mathfrak{g}$.

The Hopf superalgebra structure on $\widetilde{\mathcal{U}}$ gives a $\mathfrak{g}$-module structure on the tensor product $N_{1} \otimes N_{2}:=N_{1} \otimes_{\mathbb{C}} N_{2}$ of two $\mathfrak{g}$-modules $N_{1}, N_{2}$. The map $n_{1} \otimes n_{2} \mapsto$ $(-1)^{d\left(n_{1}\right) d\left(n_{2}\right)}$ provides the canonical $\mathfrak{g}$-isomorphism $N_{1} \otimes N_{2} \rightarrow N_{2} \otimes N_{1}$.

2.4.1. Throughout the paper we shall write "ad $\mathfrak{g}$-module" instead " $\mathfrak{g}$-module with respect to the adjoint action".

View $\widetilde{\mathcal{U}}$ as a $\mathfrak{g}$-module through the adjoint action given by

$$
(\operatorname{ad} g) u:=u g-(-1)^{d(g) d(u)} u g, \quad \forall g \in \mathfrak{g}, u \in \tilde{\mathcal{U}} .
$$

As an ad $\mathfrak{g}$-module, $\widetilde{\mathcal{U}}$ is locally finite.

For any $\mathfrak{g}$-modules $N_{1}, N_{2}$ view $\operatorname{Hom}\left(N_{1}, N_{2}\right):=\operatorname{Hom}_{\mathbb{C}}\left(N_{1}, N_{2}\right)$ as a $\mathfrak{g}$-module through the adjoint action:

$$
(\operatorname{ad} g) \psi(v)=g \psi(v)-(-1)^{d(g) d(\psi)} \psi(g v) \quad \forall g \in \mathfrak{g}, \psi \in \operatorname{Hom}\left(N_{1}, N_{2}\right) .
$$

We denote by $F\left(N_{1}, N_{2}\right)$ the locally finite part of the ad $\mathfrak{g}$-module $\operatorname{Hom}\left(N_{1}, N_{2}\right)$. Notice that $F\left(N_{1}, N_{2}\right)$ coincides with the ad $\mathfrak{g}_{0}$-locally finite part of $\operatorname{Hom}\left(N_{1}, N_{2}\right)$, since $\widetilde{\mathcal{U}}$ is a finite extension of $\mathcal{U}$.

Throughout the paper an action of $\mathfrak{g}$ on $\widetilde{\mathcal{U}}$ and on $F\left(N_{1}, N_{2}\right)$ is assumed, by default, to be the adjoint action.

For a $\mathfrak{g}$-module $N$, the natural map $\widetilde{\mathcal{U}} \rightarrow \operatorname{End}(N)$, coming from the action of $\widetilde{\mathcal{U}}$ on $N$, is an ad $\mathfrak{g}$-homomorphism and its image lies in $F(N, N)$.

2.4.2. Let $L$ be a finite-dimensional $\mathfrak{g}$-module. Equip the dual supervector space $L^{*}$ by a $\mathfrak{g}$-module structure through the antipode $S$ :

$$
g \cdot f(v):=(-1)^{d(g) d(f)} f(S(g) v)=(-1)^{d(g) d(f)} f(-g v), \forall v \in L, g \in \mathfrak{g} .
$$

We shall use the following form of the Frobenius reciprocity:

$$
\operatorname{Hom}_{\mathfrak{g}}\left(L, \operatorname{Hom}\left(N_{1}, N_{2}\right)\right) \cong \operatorname{Hom}_{\mathfrak{g}}\left(L \otimes N_{1}, N_{2}\right) \cong \operatorname{Hom}_{\mathfrak{g}}\left(N_{1}, N_{2} \otimes L^{*}\right)
$$

for any $\mathfrak{g}$-modules $N_{1}, N_{2}$ and a finite-dimensional $\mathfrak{g}$-module $L$.

2.5. The category $\widetilde{\mathcal{O}}$ and Verma modules. Denote by $\mathcal{O}$ the full subcategory of the category of $\mathfrak{g}_{0}$-modules consisting of finitely generated $\mathfrak{h}$-diagonalizable $\mathfrak{g}_{0^{-}}$ modules which are locally $\mathfrak{n}_{0}^{+}$-finite. Denote by $\widetilde{\mathcal{O}}$ the similarly defined category of $\mathfrak{g}$-modules.

Since $\mathcal{U}\left(\mathfrak{n}^{+}\right)$is finite over $\mathcal{U}\left(\mathfrak{n}_{0}^{+}\right)$, a $\mathfrak{g}$-module $N$ belongs to $\widetilde{\mathcal{O}}$ iff as a $\mathfrak{g}_{0}$-module $N$ belongs to $\mathcal{O}$. In particular, any module of category $\widetilde{\mathcal{O}}$ has a finite length. 
2.5.1. For $\lambda \in \mathfrak{h}^{*}$ denote by $\mathbb{C}_{\lambda}$ a simple $\mathfrak{b}$-module such that $\mathfrak{n}^{+} v=0$ and $h v=$ $\lambda(h) v$ for any $h \in \mathfrak{h}, v \in \mathbb{C}_{\lambda}$. Define a Verma module $\widetilde{M}(\lambda)$ by setting

$$
\widetilde{M}(\lambda):=\widetilde{\mathcal{U}} \otimes_{\mathcal{U}(\mathfrak{b})} \mathbb{C}_{\lambda} .
$$

The module $\widetilde{M}(\lambda)$ has a unique simple quotient, which we denote by $\widetilde{V}(\lambda)$. Similarly, denote by $M(\lambda)$ and $V(\lambda)$, respectively, Verma and simple $\mathfrak{g}_{0}$-modules of the highest weight $\lambda$.

2.5.2. Definition. A weight $\lambda \in \mathfrak{h}^{*}$ is called typical if $(\lambda+\rho, \beta) \neq 0$ for any isotropic $\beta \in \Delta_{1}$.

2.5.3. If $\lambda$ is typical, then $\operatorname{Ann}_{\mathcal{Z}(\mathfrak{g})} \widetilde{M}(\lambda)=\operatorname{Ann}_{\mathcal{Z}(\mathfrak{g})} \widetilde{M}\left(\lambda^{\prime}\right)$ implies $\lambda^{\prime} \in W . \lambda-$ see [K3], Theorem 2. In particular, if a typical weight $\lambda$ is a minimal element in its orbit $W \cdot \lambda$, then $\widetilde{M}(\lambda)$ is simple.

On the other hand, if a typical weight $\lambda$ is a maximal element in $W \cdot \lambda$, then $\widetilde{M}(\lambda)$ is projective in $\widetilde{\mathcal{O}}$. Indeed, take a short exact sequence $0 \rightarrow \widetilde{N}^{\prime} \rightarrow \widetilde{N} \rightarrow \widetilde{M}(\lambda) \rightarrow 0$ in $\widetilde{\mathcal{O}}$. One may assume that $\widetilde{\chi}^{k} \widetilde{N}=0$ where $\widetilde{\chi}:=\operatorname{Ann}_{\mathcal{Z}(\mathfrak{g})} \widetilde{M}(\lambda)$ and $k$ is a positive integer. Then the weight of a primitive vector of any simple subquotient of $\widetilde{N}$ belongs to $W . \lambda$. Since $\lambda$ is a maximal element of $W . \lambda$ one has $\Omega(\widetilde{N}) \cap\left\{\lambda+\mathbb{Z} \Delta^{+}\right\} \subseteq$ $\left\{\lambda-\mathbb{N} \Delta^{+}\right\}$. Thus a preimage of a highest weight vector of $\widetilde{M}(\lambda)$ is primitive and the above exact sequence splits.

By a similar argument, a short exact sequence $0 \rightarrow \widetilde{M}\left(\lambda^{\prime}\right) \rightarrow \widetilde{N} \rightarrow \widetilde{M}(\lambda) \rightarrow 0$ in $\widetilde{\mathcal{O}}$ splits if $\lambda^{\prime} \ngtr \lambda$.

2.5.4. Definition. A weight $\lambda \in \mathfrak{h}^{*}$ is called strongly typical if $(\lambda+\rho, \beta) \neq 0$ for any $\beta \in \Delta_{1}$.

2.5.5. Call the highest weight modules $\widetilde{V}(\lambda), \widetilde{M}(\lambda)$ typical (resp., strongly typical) if $\lambda$ is typical (resp., strongly typical).

Apart from the cases $B(m, n)$ (that is, $\mathfrak{o s p}(2 m+1,2 n)$ ) and $G(3)$, all odd roots of $\mathfrak{g}$ are isotropic and thus the notions of "typical" and "strongly typical" coincide.

2.5.6. Take $N \in \widetilde{\mathcal{O}}$. Equip the graded dual vector space $N^{\#}:=\bigoplus_{\mu \in \mathfrak{h}^{*}}\left(\left.N\right|_{\mu}\right)^{*}$ by the $\mathfrak{g}$-module structure

$$
\left.u . f(v):=(-1)^{d(f) d(u)} f(\sigma(u) v)\right), \quad \forall u \in \widetilde{\mathcal{U}}, f \in N^{\#}, v \in N,
$$

where $\sigma: \widetilde{\mathcal{U}} \rightarrow \widetilde{\mathcal{U}}$ is the superantiautomorphism defined in 2.3 One can easily see that $N \mapsto N^{\#}$ defines a duality functor on $\widetilde{\mathcal{O}}$.

Since the restriction of $\sigma$ on $\mathfrak{h}$ is equal to identity, $\operatorname{ch} N^{\#}=\operatorname{ch} N$. In particular, $\widetilde{V}(\lambda)^{\#} \cong \widetilde{V}(\lambda)$ and it is isomorphic to the socle of $\widetilde{M}(\lambda)^{\#}$.

2.5.7. According to $[\mathrm{LM}]$, for any Zariski dense $S \subseteq \mathfrak{h}^{*}$

$$
\bigcap_{\lambda \in S} \operatorname{Ann} \widetilde{M}(\lambda)=0
$$


2.5.8. A Verma module is not irreducible iff its highest weight is a root of a Shapovalov form. Shapovalov forms are polynomials in $\mathcal{S}(\mathfrak{h})$ indexed by the weights of $\mathcal{U}\left(\mathfrak{n}^{-}\right)$. Each polynomial admits a linear factorization which was established by Kac. The linear factors of these polynomials are

$$
\begin{array}{ll}
\alpha^{\vee}+(\rho, \alpha)-n(\alpha, \alpha) / 2, & n \in \mathbb{N}^{+}, \alpha \in \bar{\Delta}_{0}^{+}, \\
\alpha^{\vee}+(\rho, \alpha)-n(\alpha, \alpha) / 2, & n \in 2 \mathbb{N}+1, \alpha \in\left(\Delta_{1}^{+} \backslash \bar{\Delta}_{1}^{+}\right), \\
\alpha^{\vee}+(\rho, \alpha), & \alpha \in \bar{\Delta}_{1}^{+} .
\end{array}
$$

Hence $\widetilde{M}(\lambda)$ is not irreducible iff $(\lambda+\rho, \alpha)=n(\alpha, \alpha) / 2$ for a positive root $\alpha$ and a positive integer $n$ which should be odd for odd $\alpha$.

2.6. Finite-dimensional modules. Necessary and sufficient conditions for $\widetilde{V}(\lambda)$ to be finite dimensional are given in [K1], Theorem 8. One can immediately see from these conditions that any typical finite-dimensional $\widetilde{V}(\lambda)$ is strongly typical. If $\widetilde{V}(\lambda), \widetilde{V}$ are finite dimensional satisfying $\operatorname{Ann}_{\mathcal{Z}(\mathfrak{g})} \widetilde{V}(\lambda)=\operatorname{Ann}_{\mathcal{Z}(\mathfrak{g})} \widetilde{V}$ and $\widetilde{V}$ is typical, then $\widetilde{V} \cong \widetilde{V}(\lambda)$-see [K2], Proposition 2.7.

The following character formula of a typical finite-dimensional module is established by Kac (see [K2], $[\mathrm{K} 3]$ ):

$$
\operatorname{ch} \widetilde{V}(\lambda)=D \sum_{w \in W} \operatorname{sn}(w) e^{w \cdot \lambda}, \text { where } D=\prod_{\alpha \in \Delta_{0}^{+}}\left(1-e^{-\alpha}\right)^{-1} \prod_{\beta \in \Delta_{1}^{+}}\left(1+e^{-\beta}\right) .
$$

2.7. Odd reflections. The odd reflections were introduced by I. Penkov and V. Serganova- see [PS2], 3.1. If $\mathfrak{g}$ is a basic classical Lie superalgebra, the definition takes the following form. Two Borel subsuperalgebras $\mathfrak{b}, \mathfrak{b}^{\prime} \subset \mathfrak{g}$ are connected by an odd reflection along $\beta$ iff $\beta$ is a simple odd isotropic root of $\mathfrak{b}^{\prime}$ and

$$
\Delta(\mathfrak{b})=\{-\beta\} \cup \Delta\left(\mathfrak{b}^{\prime}\right) \backslash\{\beta\} .
$$

Note that $\Delta_{0}(\mathfrak{b})=\Delta_{0}\left(\mathfrak{b}^{\prime}\right)$ and so $\mathfrak{b}_{0}=\mathfrak{b}_{0}^{\prime}$. As shown in [PS2], 3.1, any Borel subsuperalgebras $\mathfrak{b}, \mathfrak{b}^{\prime} \subset \mathfrak{g}$ satisfying $\mathfrak{b}_{0}=\mathfrak{b}_{0}^{\prime}$ are connected by a chain of odd reflections.

Let $\mathfrak{b}$ and $\mathfrak{b}^{\prime}$ be Borel subsuperalgebras connected by the odd reflection along an odd isotropic root $\beta$. Then $\mathfrak{b}^{\prime}=\mathfrak{b} \cap \mathfrak{b}^{\prime}+\mathbb{C} x$ where $x$ is a non-zero element of $\mathfrak{g}_{\beta}$. If $v$ is a $\mathfrak{b}$-primitive vector such that $x v=0$, then $v$ is also $\mathfrak{b}^{\prime}$-primitive. This implies $\widetilde{V}_{\mathfrak{b}}(\lambda)=\widetilde{V}_{\mathfrak{b}^{\prime}}(\lambda)$ for $\lambda$ satisfying $(\lambda, \beta)=0$. If $x v \neq 0$, then $x v$ is $\mathfrak{b}^{\prime}$-primitive since $[x, x]=0$. This implies $\widetilde{V}_{\mathfrak{b}}(\lambda)=\widetilde{V}_{\mathfrak{b}^{\prime}}(\lambda+\beta)$ and $\widetilde{M}_{\mathfrak{b}}(\lambda)=\widetilde{M}_{\mathfrak{b}^{\prime}}(\lambda+\beta)$ for $(\lambda, \beta) \neq 0$. Remark that $\left(\rho_{\mathfrak{b}}, \beta\right)=0$, since $\beta$ is a simple isotropic root, and so $(\lambda, \beta)=0$ iff $\left(\lambda+\rho_{\mathfrak{b}}, \beta\right)=0$. Taking into account that $\rho_{\mathfrak{b}}=\rho_{\mathfrak{b}^{\prime}}+\beta$, one concludes that for Borel subsuperalgebras $\mathfrak{b}, \mathfrak{b}^{\prime}$ satisfying $\mathfrak{b}_{0}=\mathfrak{b}_{0}^{\prime}$ and a $\mathfrak{b}$-typical weight $\lambda$ one has $\widetilde{V}_{\mathfrak{b}}(\lambda)=\widetilde{V}_{\mathfrak{b}^{\prime}}\left(\lambda^{\prime}\right)$ and $\widetilde{M}_{\mathfrak{b}}(\lambda)=\widetilde{M}_{\mathfrak{b}^{\prime}}\left(\lambda^{\prime}\right)$ where $\lambda+\rho_{\mathfrak{b}}=\lambda^{\prime}+\rho_{\mathfrak{b}^{\prime}}$.

2.8. Completely reducible Lie superalgebras. Recall that a Lie superalgebra is called completely reducible if all its finite-dimensional modules are completely reducible. According to the theorem of Djoković and Hochschild (see [Sch], p. 239), any finite-dimensional completely reducible Lie superalgebra is a direct sum of semisimple Lie algebras and algebras $\mathfrak{o s p}(1,2 l)(l \geq 1)$. The superalgebra $\mathfrak{g}=$ $\mathfrak{o s p}(1,2 l)$ has many features of the semisimple Lie algebras; in particular, $\widetilde{\mathcal{U}}$ is a domain and $\mathcal{Z}(\mathfrak{g})$ is a polynomial algebra. 
2.8.1. Separation Theorem. In $\mathrm{Ko}$ Kostant establishes the following theorem which is sometimes called the "Separation Theorem".

Theorem. Let $\mathfrak{g}$ be a semisimple complex Lie algebra. There exists an ad $\mathfrak{g}$ invariant subspace $H$ in $\mathcal{U}(\mathfrak{g})$ such that the multiplication map induces an isomorphism $\mathcal{Z}(\mathfrak{g}) \otimes H \stackrel{\sim}{\longrightarrow} \mathcal{U}(\mathfrak{g})$. Moreover, for every simple finite-dimensional module $V,[H: V]=\operatorname{dim} V_{0}$.

Such an ad $\mathfrak{g}$-invariant subspace $H$ is called a harmonic space.

In [M1] Musson proves the analogous theorem for $\mathfrak{g}=\mathfrak{o s p}(1,2 l)$.

2.8.2. Annihilation Theorem. In $[\mathrm{D}]$ Duflo proves the following theorem.

Theorem. Let $\mathfrak{g}$ be a semisimple complex Lie algebra. Then for any $\lambda \in \mathfrak{h}^{*}$

$$
\operatorname{Ann} M(\lambda)=\mathcal{U}(\mathfrak{g}) \operatorname{Ann}_{\mathcal{Z}(\mathfrak{g})} M(\lambda) .
$$

Let $\mathfrak{g}$ be a semisimple complex Lie algebra and $M$ a Verma module. The multiplicity of each simple finite-dimensional module in $F(M, M)$ is equal to $\left.\operatorname{dim} V\right|_{0}$ if $M$ is a $\mathfrak{g}_{0}$-simple Verma module. Combining the above theorem and Theorem 2.8.1 one concludes that for such $M$ the natural map $\tilde{\mathcal{U}} / \operatorname{Ann} M(\lambda) \rightarrow F(M, M)$ is an isomorphism. In J1], 6.4, Joseph generalizes this result to any $\mathfrak{g}_{0}$-Verma module.

2.8.3. For the case $\mathfrak{g}=\mathfrak{o s p}(1,2 l)$ the following results are obtained in [GL1, GL2].

Theorem. The annihilator of a Verma module $\widetilde{M}$ coincides with $\widetilde{\mathcal{U}} \operatorname{Ann}_{\mathcal{Z}(\mathfrak{g})} \widetilde{M}$ iff $\widetilde{M}$ is strongly typical.

Using the Separation Theorem, one concludes that for a strongly typical $\widetilde{M}$ one has $\widetilde{\mathcal{U}} /(\operatorname{Ann} \widetilde{M}) \cong H$.

To describe the non-centrally generated annihilators of Verma modules, it is convenient to substitute the centre $\mathcal{Z}(\mathfrak{g})$ by the algebra $\widetilde{\mathcal{Z}}(\mathfrak{g})$ defined in 3.3 ,

Theorem. For any $\lambda \in \mathfrak{h}^{*}$

$$
\text { Ann } \widetilde{M}(\lambda)=\widetilde{\mathcal{U}} \operatorname{Ann}_{\tilde{\mathcal{Z}}(\mathfrak{g})} \widetilde{M}(\lambda) .
$$

If $\lambda$ is not strongly typical, then the ideal $\operatorname{Ann}_{\tilde{\mathcal{Z}}(\mathfrak{g})} \widetilde{M}(\lambda)$ is a maximal ideal of the algebra $\widetilde{\mathcal{Z}}(\mathfrak{g})$.

The algebra $\widetilde{\mathcal{U}}$ is free over $\widetilde{\mathcal{Z}}(\mathfrak{g})$; more precisely, $\widetilde{\mathcal{U}}$ contains an ad $\mathfrak{g}_{0}$-submodule $K$ such that the multiplication map induces the ad $\mathfrak{g}_{0}$-isomorphism $K \otimes \widetilde{\mathcal{Z}}(\mathfrak{g}) \rightarrow \widetilde{\mathcal{U}}$. Moreover, as ad $\mathfrak{g}_{0}$-modules $H \cong K \oplus K$ and $K \cong \widetilde{\mathcal{U}} /($ Ann $\widetilde{M})$ if $\widetilde{M}$ is not strongly typical.

\section{Category $\mathcal{F}$ in And a twisted adjoint aCtion}

3.1. Category $\mathcal{F}$ in. Denote by $\mathcal{F} i_{0}$ the full subcategory of the category of $\mathfrak{g}_{0^{-}}$ modules whose objects are sums of simple finite-dimensional modules. Denote by $\mathcal{F}$ in the full subcategory of the category $\mathfrak{g}$-modules whose objects, considered as $\mathfrak{g}_{0}$-modules, belong to $\mathcal{F}_{n_{0}}$. Since $\widetilde{\mathcal{U}}$ is a finite extension of $\mathcal{U}\left(\mathfrak{g}_{0}\right)$, any module $N \in \mathcal{F}$ in is locally finite. In other words, the objects of $\mathcal{F}$ in are the locally finite $\mathfrak{h}$-diagonalizable modules $\left(\mathfrak{g}_{0}\right.$ is reductive and so all locally finite $\mathfrak{h}$-diagonalizable modules are completely reducible). 
Denote by Irr the set of isomorphism classes of simple finite-dimensional $\mathfrak{g}$ modules and by $\operatorname{Irr}_{0}$ the set of isomorphism classes of simple finite-dimensional $\mathfrak{g}_{0}$-modules. Note that $\widetilde{\mathcal{U}}$ considered as an ad $\mathfrak{g}$-module belongs to $\mathcal{F}$ in.

Throughout this section all modules are objects of $\mathcal{F}$ in. Everywhere in the paper, injectivity and projectivity mean, by default, injectivity and projectivity in the category $\mathcal{F}$ in.

3.1.1. Recall that the socle $\operatorname{Soc} N$ of the module $N$ is the sum of its simple submodules. Since any module in $\mathcal{F}$ in is locally finite, it has a finite-dimensional submodule and so a non-trivial socle.

Recall that a module $N$ is called an essential extension of its submodule $N^{\prime}$ if for any non-zero submodule $N^{\prime \prime}$ of $N$ one has $N^{\prime} \cap N^{\prime \prime} \neq 0$. Any module in $\mathcal{F}$ in is an essential extension of its socle.

3.1.2. For a homomorphism $\psi: N \rightarrow N^{\prime}$ denote by Soc $\psi$ its restriction to $\operatorname{Soc} N$. If $\operatorname{Soc} \psi$ is a monomorphism, then $\psi$ is also a monomorphism, since ker $\psi \cap \operatorname{Soc} N=0$ and $N$ is an essential extension of $\operatorname{Soc} N$.

3.2. Injective and projective objects in $\mathcal{F}$ in. Recall that an injective envelope $\mathrm{E}(N)$ of the module $N$ is an injective module which is an essential extension of $N$. An injective envelope of a given module is unique up to isomorphism.

A typical simple finite-dimensional module is injective and projective in $\mathcal{F}$ in (see 2.5.2).

A standard reasoning of $\mathrm{McL}, 3.11$, shows that any submodule $N$ of an injective module $E$ has an injective envelope which is a direct summand of $E$. In Lemma 3.2.2 we show that any module in $\mathcal{F}$ in has an injective envelope.

3.2.1. A finite-dimensional $\mathfrak{g}_{0}$-module $L$ is projective and injective in $\mathcal{F} \operatorname{in}_{0}$ and so $\operatorname{Coind}_{\mathfrak{g}_{0}}^{\mathfrak{g}} L \cong \operatorname{Ind}_{\mathfrak{g}_{0}}^{\mathfrak{g}} L$ is projective and injective in $\mathcal{F}$ in - see 2.3.5.

Let $\widetilde{V}$ be a simple finite-dimensional $\mathfrak{g}$-module and $V$ its simple $\mathfrak{g}_{0}$-submodule. Then $\widetilde{V}$ is a submodule of the injective module $\operatorname{Coind}_{\mathfrak{g}_{0}}^{\mathfrak{g}} V$. Therefore $\widetilde{V}$ has an injective envelope $\mathrm{E}(\widetilde{V})$ which is a direct summand of $\operatorname{Coind}_{\mathfrak{g}_{0}}^{\mathfrak{g}} V$. The last is projective and so $\mathrm{E}(\widetilde{V})$ is projective as well.

3.2.2. Lemma. Any module $N \in \mathcal{F}$ in has an injective envelope $\mathrm{E}(N)$. Moreover $\mathrm{E}(N) \cong \mathrm{E}(\operatorname{Soc} N)$.

Proof. By 3.1.1 $N$ is an essential extension of Soc $N$. Therefore, by [McL], 3.11.1, there exists a monomorphism $\iota: N \rightarrow \mathrm{E}(\operatorname{Soc} N)$ whose socle is the natural embed$\operatorname{ding} \operatorname{Soc} N \rightarrow \mathrm{E}(\operatorname{Soc} N)$. Since $\mathrm{E}(\operatorname{Soc} N)$ is an essential extension of $\operatorname{Soc} N$, it is also an essential extension of $N$. Hence $\mathrm{E}(\operatorname{Soc} N)$ is an injective envelope of $N$.

\subsubsection{Lemma. Any direct sum of injective modules in $\mathcal{F}$ in is injective.}

Proof. Let $E_{i}, i \in I$, be a collection of injective modules. One has to check that for any monomorphism $\iota: A \rightarrow B$ and any homomorphism $\phi: A \rightarrow \bigoplus_{i \in I} E_{i}$ there exists a homomorphism $\psi: B \rightarrow \bigoplus_{i \in I} E_{i}$ such that $\psi \iota=\phi$. By a standard reasoning based on Zorn's lemma, it is enough to verify the above assertion assuming $B$ is cyclic. Any cyclic module in $\mathcal{F}$ in is finite dimensional. If $B$ is finite dimensional, then $A$ is also finite dimensional and so $\phi(A)$ lies in a finite subsum $\bigoplus_{i \in J} E_{i}(J$ is a finite subset of $I$ ). Since each $E_{i}$ is injective, $\bigoplus_{i \in J} E_{i}$ is injective and so there 
exists $\psi: B \rightarrow \bigoplus_{i \in J} E_{i}$ such that $\psi \iota(a)=\phi(a)$ for all $a \in A$. The assertion follows.

\subsubsection{Corollary.}

$$
\mathrm{E}\left(\bigoplus_{i \in I} N_{i}\right)=\bigoplus_{i \in I} \mathrm{E}\left(N_{i}\right) .
$$

3.2.5. Proposition. Any injective module in $\mathcal{F}$ in is projective.

Proof. Take an injective module $N$ and write $\operatorname{Soc} N=\sum_{i \in I} L_{i}$ where the $L_{i}$ are simple. Combining Lemma 3.2 .2 and Corollary 3.2.4, one obtains $N \cong \bigoplus_{i \in I} \mathrm{E}\left(L_{i}\right)$. By 3.2.1, all $\mathrm{E}\left(L_{i}\right)$ are projective so $N$ is also projective.

3.3. Twisted adjoint action. In this subsection we present some results from G1] which are used in the sequel.

Define a twisted adjoint action of $\mathfrak{g}$ on $\widetilde{\mathcal{U}}$ by setting

$$
\left(\operatorname{ad}^{\prime} g\right) u=g u-(-1)^{d(g)(d(u)+1)} u g, \forall g \in \mathfrak{g}, u \in \widetilde{\mathcal{U}} .
$$

Note that $\operatorname{ad} g=\operatorname{ad}^{\prime} g$ for $g \in \mathfrak{g}_{0}$.

The anticentre $\mathcal{A}(\mathfrak{g})$ is the set of invariants of $\widetilde{\mathcal{U}}$ under the twisted adjoint action $\operatorname{ad}^{\prime} \mathfrak{g}$. The product of two anticentral elements is central. For $\mathfrak{g}$ a basic classical Lie superalgebra, $\mathcal{A}(\mathfrak{g})$ is even and so any anticentral element commutes with the even elements of $\widetilde{\mathcal{U}}$ and anticommutes with the odd ones. Therefore "the ghost centre"

$$
\widetilde{\mathcal{Z}}(\mathfrak{g}):=\mathcal{Z}(\mathfrak{g})+\mathcal{A}(\mathfrak{g})
$$

is a commutative subalgebra of $\widetilde{\mathcal{U}}$.

For $\mathfrak{g}=\mathfrak{o s p}(1,2 l), \widetilde{\mathcal{Z}}(\mathfrak{g})$ is a polynomial algebra and, moreover, $\widetilde{\mathcal{Z}}(\mathfrak{g})=\mathcal{Z}(\mathfrak{g}) \oplus$ $T \mathcal{Z}(\mathfrak{g})$ where $T$ is the element defined in 3.3.2

3.3.1. Let $L$ be an ad $\mathfrak{g}_{0}$-submodule of $\mathcal{U}$. Then the $\operatorname{ad}^{\prime} \mathfrak{g}$-submodule generated by $L$ in $\widetilde{\mathcal{U}}$ is isomorphic to the induced module $\operatorname{Ind}_{\mathfrak{g}_{0}}^{\mathfrak{g}} L$. As an $\operatorname{ad}^{\prime} \mathfrak{g}$-module $\widetilde{\mathcal{U}}$ is generated by $\mathcal{U}$ and so $\widetilde{\mathcal{U}} \cong \operatorname{Ind}_{\mathfrak{g}_{0}}^{\mathfrak{g}} \mathcal{U}$. Using the isomorphism $\operatorname{Ind}_{\mathfrak{g}_{0}}^{\mathfrak{g}} \mathcal{U} \cong \operatorname{Coind}_{\mathfrak{g}_{0}}^{\mathfrak{g}} \mathcal{U}$, one obtains a linear isomorphism $\mathcal{Z}\left(\mathfrak{g}_{0}\right) \stackrel{\sim}{\longrightarrow} \mathcal{A}(\mathfrak{g})$ given by $z \mapsto\left(\operatorname{ad}^{\prime} u\right) z$ where $u \in \widetilde{\mathcal{U}}$ is an element such that $u V(0)$ is the trivial $\mathfrak{g}$-submodule of $\operatorname{Ind}_{\mathfrak{g}_{0}}^{\mathfrak{g}} V(0)$.

An anticentral element $z$ acts on a module generated by a primitive vector $v$ in the following way. It acts as $\mathcal{P}(z)(\lambda)$ id on $\widetilde{\mathcal{U}}_{0} v$ and as $\left(-\mathcal{P}(z)(\lambda)\right.$ id) on $\widetilde{\mathcal{U}}_{1} v$ $\left(\widetilde{\mathcal{U}}_{0}, \widetilde{\mathcal{U}}_{1}\right.$ are homogeneous components of $\left.\widetilde{\mathcal{U}}\right)$. From 2.5.7 it follows that the HarishChandra projection provides a monomorphism $\mathcal{A}(\mathfrak{g}) \rightarrow \mathcal{S}(\mathfrak{h})^{W}$. The image of this monomorphism is equal to $t \mathcal{S}(\mathfrak{h})^{W}$. where

$$
t:=\prod_{\beta \in \Delta_{1}^{+}}\left(\beta^{\vee}+(\rho, \beta)\right) .
$$

Any non-zero element of $\mathcal{A}(\mathfrak{g})$ is a non-zero divisor in $\widetilde{\mathcal{U}}$.

3.3.2. Denote by $T$ the element of $\mathcal{A}(\mathfrak{g})$ such that $\mathcal{P}(T)=t$. Remark that a Verma module is strongly typical iff its annihilator does not contain $T$.

Up to a non-zero scalar, $T$ is equal to the image of 1 under the above linear isomorphism $\mathcal{Z}\left(\mathfrak{g}_{0}\right) \stackrel{\sim}{\longrightarrow} \mathcal{A}(\mathfrak{g})$. The image of $T$ in the symmetric algebra is a nonzero element of the one-dimensional vector space $\Lambda^{\text {top }} \mathfrak{g}_{1}$. 
Remark that $T \mathcal{A}(\mathfrak{g}) \subset \mathcal{Z}(\mathfrak{g})$ and the restriction of the Harish-Chandra projection provides an isomorphism $T \mathcal{A}(\mathfrak{g}) \stackrel{\sim}{\longrightarrow} t^{2} \mathcal{S}(\mathfrak{h})^{W}$. For $\mathfrak{g}=\mathfrak{o} \mathfrak{p} \mathfrak{p}(1,2 l)$, this implies that $\mathcal{A}(\mathfrak{g})$ is a free module over $\mathcal{Z}(\mathfrak{g})$ generated by $T$.

It is easy to check that $(\operatorname{ad} g)(u T)=\left(\left(\operatorname{ad}^{\prime} g\right) u\right) T$. Since $T$ is a non-zero divisor, the ideal $\widetilde{\mathcal{U}} T$ considered as an ad $\mathfrak{g}$-module is isomorphic to $\widetilde{\mathcal{U}}$ considered as an $\operatorname{ad}^{\prime} \mathfrak{g}$-module. Hence as ad $\mathfrak{g}$-module $\widetilde{\mathcal{U}} T \cong \operatorname{Ind}_{\mathfrak{g}_{0}}^{\mathfrak{g}} \mathcal{U}$ and, in particular, it is injective in $\mathcal{F}$ in. Note that $\widetilde{\mathcal{U}} T$ is a two-sided ideal. Remark that, apart from the case when $\mathfrak{g}$ is completely reducible, $\widetilde{\mathcal{U}}$ itself as an ad $\mathfrak{g}$-module is neither injective nor projective in $\mathcal{F}$ in: it contains, as a direct summand, a trivial representation generated by 1 .

\subsubsection{For any $N \in \mathcal{F}$ in $_{0}$ one has}

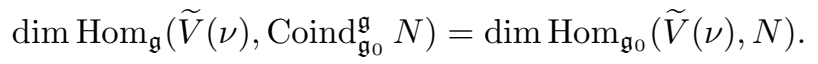

Therefore

$$
\operatorname{Ind}_{\mathfrak{g}_{0}}^{\mathfrak{g}} N \cong \operatorname{Coind}_{\mathfrak{g}_{0}}^{\mathfrak{g}} N=\bigoplus_{\widetilde{V} \in \operatorname{Irr}} \mathrm{E}(\widetilde{V})^{r(\widetilde{V})}, \quad r(\widetilde{V}):=\operatorname{dim}\left(\operatorname{Hom}_{\mathfrak{g}_{0}}(\widetilde{V}, N)\right) .
$$

This has the following useful consequence. Let $H$ be a harmonic space of $\mathcal{U}\left(\mathfrak{g}_{0}\right)$ that is an ad $\mathfrak{g}_{0}$-submodule of $\mathcal{U}\left(\mathfrak{g}_{0}\right)$ such that the multiplication map provides an isomorphism $H \otimes \mathcal{Z}\left(\mathfrak{g}_{0}\right) \rightarrow \mathcal{U}\left(\mathfrak{g}_{0}\right)$ - see2.8.1 Then $H \cong \bigoplus_{V \in \operatorname{Irr} r_{0}} V^{\left.\oplus \operatorname{dim} V\right|_{0}}$. Taking into account 3.3 .1 and (2), one obtains

$$
\left(\operatorname{ad}^{\prime} \tilde{\mathcal{U}}\right) H \cong \operatorname{Ind}_{\mathfrak{g}_{0}}^{\mathfrak{g}} H=\bigoplus_{V \in \operatorname{Irr}_{0}} \operatorname{Coind}_{\mathfrak{g}_{0}}^{\mathfrak{g}} V^{\left.\oplus \operatorname{dim} V\right|_{0}}=\bigoplus_{\widetilde{V} \in \operatorname{Irr}} \mathrm{E}(\widetilde{V})^{\oplus \operatorname{dim} \widetilde{V} l_{0}}
$$

since $\left.\sum_{V \in \operatorname{Irr}_{0}} \operatorname{dim} V\right|_{0} \cdot \operatorname{dim}\left(\operatorname{Hom}_{\mathfrak{g}_{0}}(\widetilde{V}, V)\right)=\left.\operatorname{dim} \widetilde{V}\right|_{0}$ for any $\widetilde{V} \in \operatorname{Irr}$.

\section{The ad $\mathfrak{g}$-Module Structure of $\tilde{\mathcal{U}}$}

In this section we study the ad $\mathfrak{g}$-module structure of $\widetilde{\mathcal{U}}$. We start by studying $\operatorname{Hom}_{\mathfrak{g}}(\widetilde{V}, \widetilde{\mathcal{U}})$ for a simple finite-dimensional module $\widetilde{V}$. For each $\widetilde{V}$ we construct a central element $z$ such that the localized module $\operatorname{Hom}_{\mathfrak{g}}\left(\widetilde{V}, \widetilde{\mathcal{U}}\left[z^{-1}\right]\right)=$ $\operatorname{Hom}_{\mathfrak{g}}(\widetilde{V}, \widetilde{\mathcal{U}})\left[z^{-1}\right]$ is a free $\mathcal{Z}(\mathfrak{g})\left[z^{-1}\right]$-module whose rank is equal to $\left.\operatorname{dim} \widetilde{V}\right|_{0}$. In 4.6 we define and study PRV determinants for non-completely reducible basic classical Lie superalgebras.

In 4.7 we show that for a suitable $S \subset \mathcal{Z}(\mathfrak{g})$ the localized algebra $\widetilde{\mathcal{U}}\left[S^{-1}\right]$ is free over its centre $\mathcal{Z}(\mathfrak{g})\left[S^{-1}\right]$ and that the corresponding harmonic space $H$ ("generic harmonic space") is an injective (in $\mathcal{F}$ in) ad $\mathfrak{g}$-module. Moreover, the multiplicity of a simple finite-dimensional module $\widetilde{V}$ in $\operatorname{Soc} H$ is equal to $\left.\operatorname{dim} \widetilde{V}\right|_{0}$.

4.1. The following lemma of A. Joseph and G. Letzter provides a connection between $\operatorname{Hom}_{\mathfrak{g}}(\widetilde{V}, \widetilde{\mathcal{U}})$ and $\operatorname{Hom}\left(\left.\widetilde{V}\right|_{0}, \mathcal{S}(\mathfrak{h})\right)$.

4.1.1. Lemma. An ad $\mathfrak{g}$-submodule $N$ of $\widetilde{\mathcal{U}}$ lies in the annihilator of $\widetilde{V}(\lambda)$ iff

$$
\mathcal{P}\left(\left.N\right|_{0}\right)(\lambda)=0 .
$$

Proof. The proof is the same as in [J3], 7.2. Remark that $\mathcal{P}(N)=\mathcal{P}\left(\left.N\right|_{0}\right)$. Let $v_{\lambda}$ be a highest weight vector of $\widetilde{V}(\lambda)$ and $\widetilde{V}(\lambda)_{-}:=\mathcal{U}\left(\mathfrak{n}^{-}\right) \mathfrak{n}^{-} v$. One has

$$
\mathcal{P}(N)(\lambda)=0 \Longleftrightarrow N v_{\lambda} \subseteq \widetilde{V}(\lambda)_{-} .
$$

In particular, $N \widetilde{V}(\lambda)=0$ forces $\mathcal{P}(N)(\lambda)=0$. 
For the inverse implication, assume that $\mathcal{P}\left(\left.N\right|_{0}\right)(\lambda)=0$, that is, $N v_{\lambda} \subseteq \widetilde{V}(\lambda)_{-}$. The ad $\mathfrak{g}$-invariance of $N$ implies $\mathcal{U}\left(\mathfrak{n}^{-}\right) N=N \mathcal{U}\left(\mathfrak{n}^{-}\right)$and thus

$$
N \widetilde{V}(\lambda)=N \mathcal{U}\left(\mathfrak{n}^{-}\right) v_{\lambda}=\mathcal{U}\left(\mathfrak{n}^{-}\right) N v_{\lambda} \subseteq \mathcal{U}\left(\mathfrak{n}^{-}\right) \widetilde{V}(\lambda)_{-} \subseteq \widetilde{V}(\lambda)_{-} \underset{\neq}{\subseteq}(\lambda) .
$$

The ad $\mathfrak{g}$-invariance of $N$ implies also $\mathcal{U}(\mathfrak{g}) N=N \mathcal{U}(\mathfrak{g})$ and so $N \widetilde{V}(\lambda)$ is a submodule of $\widetilde{V}(\lambda)$. Hence $N \widetilde{V}(\lambda)=0$ as required.

4.1.2. Corollary. Take $\widetilde{V} \in \operatorname{Irr}$, and let $v_{1}, \ldots, v_{r}$ be a basis of $\left.\widetilde{V}\right|_{0}$ and $\lambda \in \mathfrak{h}^{*}$. For any $\theta_{1}, \ldots, \theta_{k} \in \operatorname{Hom}_{\mathfrak{g}}(\widetilde{V}, \widetilde{\mathcal{U}})$, the image of the space $\sum_{i=1}^{k} \theta_{i}(\widetilde{V})$ under the natural map $\widetilde{\mathcal{U}} \rightarrow \operatorname{End}(\widetilde{V}(\lambda))$ is isomorphic to $\widetilde{V}^{\oplus m}$ where $m$ is the rank of the $\operatorname{matrix}\left(\mathcal{P}\left(\theta_{j}\left(v_{i}\right)\right)(\lambda)\right)_{i=1, r}^{j=1, k}$.

4.1.3. Combining 2.5.7 and Lemma 4.1.1 one concludes that for an ad $\mathfrak{g}$-submodule $N$ of $\widetilde{\mathcal{U}}$ the equality $\mathcal{P}\left(\left.N\right|_{0}\right)=0$ implies $N=0$. Moreover $N=0$ provided that $\mathcal{P}\left(\left.N\right|_{0}\right)(R)=0$ for a Zariski dense subset $R$ of $\mathfrak{h}^{*}$.

4.2. Notation. Fix $\tilde{V} \in \operatorname{Irr}$ and consider $\operatorname{Hom}_{\mathfrak{g}}(\widetilde{V}, \widetilde{\mathcal{U}})$ as a $\mathcal{Z}(\mathfrak{g})$-module with respect to the action induced by the multiplication.

The Harish-Chandra projection induces the map

$$
\Psi: \operatorname{Hom}_{\mathfrak{g}}(\widetilde{V}, \widetilde{\mathcal{U}}) \rightarrow \operatorname{Hom}\left(\left.\widetilde{V}\right|_{0}, \mathcal{S}(\mathfrak{h})\right)
$$

given by

$$
\Psi(\phi)(v)=\mathcal{P}(\phi(v)), \forall \phi \in \operatorname{Hom}_{\mathfrak{g}}(\widetilde{V}, \widetilde{\mathcal{U}}),\left.v \in \widetilde{V}\right|_{0}
$$

This map is a monomorphism by 4.1.3 Denote by $\mathcal{I}(\widetilde{V})$ the image of $\Psi$.

Define the action of $\mathcal{Z}(\mathfrak{g})$ on $\mathcal{S}(\mathfrak{h})$ by setting $z p:=\mathcal{P}(z) p$. This action induces the structure of a $\mathcal{Z}(\mathfrak{g})$-module on $\operatorname{Hom}\left(\left.\widetilde{V}\right|_{0}, \mathcal{S}(\mathfrak{h})\right)$. Obviously, $\Psi$ is a $\mathcal{Z}(\mathfrak{g})$-map. Thus the study of the $\mathcal{Z}(\mathfrak{g})$-module structure of $\operatorname{Hom}_{\mathfrak{g}}(\widetilde{V}, \widetilde{\mathcal{U}})$ reduces to the study of the $\mathcal{Z}(\mathfrak{g})$-module structure of $\mathcal{I}(\widetilde{V})$.

The vector space $\operatorname{Hom}\left(\left.\widetilde{V}\right|_{0}, \mathcal{S}(\mathfrak{h})\right)$ has the natural structure of an $\mathcal{S}(\mathfrak{h})$-module. We denote by $\mathcal{I}(\widetilde{V}) \mathcal{S}(\mathfrak{h})$ the $\mathcal{S}(\mathfrak{h})$-span of $\mathcal{I}(\widetilde{V})$ inside $\operatorname{Hom}\left(\left.\widetilde{V}\right|_{0}, \mathcal{S}(\mathfrak{h})\right)$.

\subsection{Examples.}

4.3.1. Example. Let $\mathfrak{g}$ be a completely reducible simple Lie superalgebra. In this case $\mathcal{P}(\mathcal{Z}(\mathfrak{g}))=\mathcal{S}(\mathfrak{h})^{W}$. The Separation Theorem (see 2.8.1) states the existence of an ad $\mathfrak{g}$-submodule $\mathcal{H}$ of $\widetilde{\mathcal{U}}$ such that the multiplication map induces the isomorphism $\mathcal{H} \otimes \mathcal{Z}(\mathfrak{g}) \stackrel{\sim}{\longrightarrow} \widetilde{\mathcal{U}}$. Thus for any $\widetilde{V} \in$ Irr, a basis of the vector space $\operatorname{Hom}_{\mathfrak{g}}(\widetilde{V}, \mathcal{H})$ is a free $\mathcal{Z}(\mathfrak{g})$-basis of $\operatorname{Hom}_{\mathfrak{g}}(\widetilde{V}, \widetilde{\mathcal{U}})$. Consequently, $\operatorname{Hom}_{\mathfrak{g}}(\widetilde{V}, \widetilde{\mathcal{U}})$ is a free $\mathcal{Z}(\mathfrak{g})$-module of the $\operatorname{rank} \operatorname{dim} \operatorname{Hom}_{\mathfrak{g}}(\widetilde{V}, \mathcal{H})=\left.\operatorname{dim} \widetilde{V}\right|_{0}$ (see 2.8.1).

4.3.2. Example. Consider the case $\mathfrak{g}=\mathfrak{s l}(2,1)$. Then $\mathfrak{h}=\operatorname{span}\{z, h\}$ where $z$ is a central element of the reductive algebra $\mathfrak{g}_{0}=\mathfrak{s l}(2) \times \mathbb{C}$ and $h$ is an element of the Cartan subalgebra of $\mathfrak{s l}(2)$. Then $\Delta_{0}=\{ \pm \alpha\}, \Delta_{1}=\{ \pm \beta ; \pm(\alpha+\beta)\}$. Choose a triangular decomposition such that $\Delta^{+}=\{\alpha, \beta, \alpha+\beta\}$. The subalgebras $\mathfrak{n}_{1}^{ \pm}=$ $\mathfrak{g}_{1} \cap \mathfrak{n}^{ \pm}$are supercommutative and are ad $\mathfrak{g}_{0}$-submodules of $\mathfrak{g}$. Let $\left\{x_{1}, x_{2}\right\}$ (resp., $\left.\left\{y_{1}, y_{2}\right\}\right)$ be a basis of $\mathfrak{n}_{1}^{+}$(resp., $\mathfrak{n}_{1}^{-}$).

The algebra $\mathcal{S}(\mathfrak{h})^{W}$. is a polynomial algebra generated by $z$ and $t=(z-h)$. $(z+h+2)$. The image $\mathcal{P}(\mathcal{Z}(\mathfrak{g}))$ in $\mathcal{S}(\mathfrak{h})^{W}$. is spanned by 1 and the elements $\left\{t^{n} z^{k}, n>0, k \geq 0\right\}$. 
Denote by $v$ a highest weight vector of $\widetilde{V}:=\widetilde{V}(\alpha+2 \beta)$; one has $\left.\widetilde{V}\right|_{0}=\mathbb{C} y_{1} y_{2} v$. It is easy to see that a highest weight vector of any copy of $\widetilde{V}$ inside $\widetilde{\mathcal{U}}$ is of the form $u x_{1} x_{2}$ where $u \in \mathcal{Z}\left(\mathfrak{g}_{0}\right)$. Up to a scalar,

$$
\mathcal{P}\left(\left(\operatorname{ad} y_{1} y_{2}\right)\left(u x_{1} x_{2}\right)\right)=(z-h)(z+h+2) \mathcal{P}(u)=t \mathcal{P}(u) .
$$

One has $\mathcal{P}\left(\mathcal{Z}\left(\mathfrak{g}_{0}\right)\right)=\mathcal{S}(\mathfrak{h})^{W}$. and so $\mathcal{I}(\widetilde{V})=\operatorname{Hom}\left(\mathbb{C}, \mathcal{S}(\mathfrak{h})^{W \cdot} \cdot t\right)$. Since $\mathcal{S}(\mathfrak{h})^{W}$ is not free over $\mathcal{P}(\mathcal{Z}(\mathfrak{g}))$, the $\mathcal{Z}(\mathfrak{g})$-module $\mathcal{I}(\widetilde{V}) \cong \operatorname{Hom}_{\mathfrak{g}}(\widetilde{V}, \widetilde{\mathcal{U}})$ is not free.

One might expect from the above example that $\mathcal{I}(\widetilde{V})$ is stable with respect to the multiplication on $\mathcal{S}(\mathfrak{h})^{W}$. However $\mathcal{I}(\widetilde{V}(0))=\operatorname{Hom}(\mathbb{C}, \mathcal{P}(\mathcal{Z}(\mathfrak{g})))$ is not stable with respect to the multiplication on $\mathcal{S}(\mathfrak{h})^{W}$. apart from the case when $\mathcal{P}(\mathcal{Z}(\mathfrak{g}))=$ $\mathcal{S}(\mathfrak{h})^{W}$.

4.4. In this subsection we show that the $\mathcal{Z}(\mathfrak{g})$-rank of the module $\operatorname{Hom}_{\mathfrak{g}}(\tilde{V}, \tilde{\mathcal{U}})$ is not greater than $\left.\operatorname{dim} \widetilde{V}\right|_{0}$. The crucial point is Lemma 4.4 .2 asserting that any elements of $\mathcal{I}(\widetilde{V})$ which are "linearly dependent" over $\mathcal{S}(\mathfrak{h})$ are "linearly dependent" over $\mathcal{Z}(\mathfrak{g})$. Throughout this subsection $\widetilde{V} \in \operatorname{Irr}$ is fixed.

4.4.1. Basic definitions. Let $A$ be a commutative domain. For an $A$-module $N$ define an $A$-rank of $N$ to be the dimension over the field of fractions Fract $A$ of the localized module $N \otimes_{A}$ Fract $A$. Call elements $\theta_{1}, \ldots, \theta_{k} \in N$ A-linearly independent if their images in the localized module $N \otimes_{A}$ Fract $A$ are linearly independent. Call the elements $A$-linearly dependent if they are not $A$-linearly independent. Call an $A$-basic system of $N$ a collection $\theta_{1}, \ldots, \theta_{k} \in N$ such that the image of this collection in the localized module $N \otimes_{A}$ Fract $A$ forms its Fract $A$-basis.

Let $L$ be a vector space, and let $v_{1}, \ldots, v_{r}$ be a basis of $L$. Let $N$ be an $A$ submodule of $\operatorname{Hom}(L, A)$ and $k$ the $A$-rank of $N$. The collection $\theta_{1}, \ldots, \theta_{k} \in N$ is an $A$-basic system of $N$ iff the matrix $\left(\theta_{j}\left(v_{i}\right)\right)_{i=1, r}^{j=1, k} \in \mathcal{M a t}_{(r \times k)}(A)$ has a $(k \times k)$ non-zero minor. Call such a non-zero minor (which is an element of $A$ ) a minor of a basic system $\theta_{1}, \ldots, \theta_{k}$.

If $p \in A$ is a minor of a basic system $\theta_{1}, \ldots, \theta_{k}$, then for any $\theta \in N$ one has $p \theta=\sum_{j=1}^{k} p_{j} \theta_{j}$ for a certain (unique) collection $p_{1}, \ldots, p_{k} \in A$. In particular, the localized module $N \otimes_{A} A\left[p^{-1}\right]$ is a free $A\left[p^{-1}\right]$-module and the images of $\theta_{1}, \ldots, \theta_{k}$ form a basis of this free module.

Recall that $t^{2} \mathcal{S}(\mathfrak{h})^{W} \subseteq \mathcal{P}(\mathcal{Z}(\mathfrak{g}))$ (see 3.3.2).

4.4.2. Lemma. (i) Elements of $\mathcal{I}(\widetilde{V})$ are $\mathcal{Z}(\mathfrak{g})$-linearly independent iff they are $\mathcal{S}(\mathfrak{h})$-linearly independent.

(ii) If $\theta_{1}, \ldots, \theta_{s} \in \mathcal{I}(\widetilde{V})$ are $\mathcal{S}(\mathfrak{h})$-linearly independent and

$$
\sum_{j=0}^{s} p_{j} \theta_{j}=0
$$

for some $\theta_{0} \in \mathcal{I}(\widetilde{V})$ and $p_{0}, \ldots, p_{s} \in \mathcal{S}(\mathfrak{h})$, then there exists $z_{0}, \ldots, z_{s} \in \mathcal{Z}(\mathfrak{g})$ such that $\sum_{j=0}^{s} z_{j} \theta_{j}=0$ and $\mathcal{P}\left(z_{0}\right)=t^{2} q$ where $q$ is a maximal $W$-invariant divisor of $p_{0}^{|W|}$.

Proof. Obviously, elements of $\mathcal{I}(\widetilde{V})$ are $\mathcal{Z}(\mathfrak{g})$-linearly independent provided that they are $\mathcal{S}(\mathfrak{h})$-linearly independent. The inverse implication follows from (ii) because we always can choose a minimal (with respect to the inclusion) subset of $\mathcal{S}(\mathfrak{h})$-linearly dependent elements in a set of $\mathcal{S}(\mathfrak{h})$-linearly dependent elements. 
Let us prove (ii). The equality $\sum_{j=0}^{s} p_{j} \theta_{j}=0$ means that for any $\mu \in \mathfrak{h}^{*}$ one has

$$
\left.\forall v \in \widetilde{V}\right|_{0} \quad 0=\sum_{j=0}^{s} p_{j}(\mu) \theta_{j}(v)(\mu)=\sum_{j=0}^{s} p_{j}(\mu) \mathcal{P}\left(\left(\Psi^{-1} \theta_{j}\right)(v)\right)(\mu) .
$$

In light of Lemma 4.1.1, this gives

$$
\forall \mu \in \mathfrak{h}^{*} \quad\left(\sum_{j=0}^{s} p_{j}(\mu) \Psi^{-1}\left(\theta_{j}\right)\right)(\widetilde{V}) \subset \operatorname{Ann} \tilde{V}(\mu) ;
$$

here the sum belongs to $\operatorname{Hom}_{\mathfrak{g}}(\widetilde{V}, \widetilde{\mathcal{U}})$ because $\Psi^{-1}\left(\theta_{j}\right) \in \operatorname{Hom}_{\mathfrak{g}}(\widetilde{V}, \widetilde{\mathcal{U}})$ and $p_{j}(\mu) \in$ $\mathbb{C}$.

Fix a root $\alpha \in \pi_{0}$; let $s_{\alpha} \in W$ be the corresponding reflection. Assume that $\mu \in \mathfrak{h}^{*}$ is such that $\widetilde{V}(\mu)=\widetilde{M}(\mu) / \widetilde{M}\left(s_{\alpha} \cdot \mu\right)$. Then, for "sufficiently large" $\mu$, any copy of $\widetilde{V}$ inside $\widetilde{\mathcal{U}}$ which annihilates $\widetilde{V}(\mu)$, also annihilates $\widetilde{M}(\mu)$ and so $\widetilde{V}\left(s_{\alpha} \cdot \mu\right)$. To be more precise, choose $\omega \in \mathbb{Q} \Delta$ such that $\left(\omega, \alpha^{\prime}\right) \geq 0$ for all $\alpha^{\prime} \in \Delta^{+}$and $(\omega, \alpha)=1$. Set

$$
\begin{aligned}
R:=\left\{\mu \in \mathfrak{h} \mid \text { (a) } n(\mu) \in \mathbb{N}^{+}, \text {(b) } n(\mu)>\right. & (-\nu, \omega), \\
& \text { (c) } \left.\forall \beta \in \Delta^{+} \backslash\{\mathbb{Q} \alpha\} \quad(\mu+\rho, \beta) \notin \mathbb{Q}\right\}
\end{aligned}
$$

where $\nu$ stands for the lowest weight of $\widetilde{V}$ and $n(\mu):=2(\mu+\rho, \alpha) /(\alpha, \alpha)$. It is easy to see that $R$ is Zariski dense in $\mathfrak{h}^{*}$ and that any element in $R$ is typical. Take $\mu \in R$. By 2.5.8, $\widetilde{M}(\mu)$ is not simple because $n(\mu) \in \mathbb{N}^{+}$. If $\widetilde{M}\left(\mu^{\prime}\right)$ is a subquotient of $\widetilde{M}(\mu)$, then $\left(\mu^{\prime}-\mu\right) \in \mathbb{N} \Delta^{-}$and, by 2.5.3. $\mu^{\prime}=w . \mu$ for some $w \in W$. Using the fact that any $w \in W$ can be written as the product $s_{\beta_{1}} \cdots s_{\beta_{m}}$ where $\beta_{1}, \ldots, \beta_{m} \in \Delta_{0}^{+}$are linearly independent (see, for example, A.1.18 of [J]2]), it is easy to deduce from condition (c) that $(w \cdot \mu-\mu) \notin \mathbb{N} \Delta^{-}$for any $w \neq$ id, $s_{\alpha}$. Thus $\widetilde{V}(\mu)=\widetilde{M}(\mu) / \widetilde{M}\left(s_{\alpha} \cdot \mu\right)$ and $\widetilde{M}\left(s_{\alpha} \cdot \mu\right)$ is simple. Observe that $s_{\alpha} \cdot \mu=\mu-n(\mu) \alpha$ and so for any $\xi \in \Omega\left(\widetilde{M}\left(s_{\alpha} \cdot \mu\right)\right)$ one has $(\xi, \omega) \leq(\mu, \omega)-n(\mu)$. Thus $(\mu+\nu) \notin$ $\Omega\left(\widetilde{M}\left(s_{\alpha} \cdot \mu\right)\right)$ due to condition $(\mathrm{a})$.

Let $N$ be a copy of $\widetilde{V}$ inside Ann $\widetilde{V}(\mu)$. Let $u_{\nu}$ be a lowest weight vector of $N$, and let $v_{\mu}$ be a highest weight vector of $\widetilde{M}(\mu)$. Since $u_{\nu} \widetilde{V}(\mu)=0$, the vector $u_{\nu} v_{\mu}$ belongs to $\widetilde{M}\left(s_{\alpha} \cdot \mu\right)$. However the latter does not have non-zero elements of weight $(\mu+\nu)$. Thus $u_{\nu} v_{\mu}=0$. One has $u_{\nu} \widetilde{M}(\mu)=u_{\nu} \mathcal{U}\left(\mathfrak{n}^{-}\right) v_{\mu}=\mathcal{U}\left(\mathfrak{n}^{-}\right) u_{\nu} v_{\mu}$ because $u_{\nu}$ is a lowest weight vector, that is, $\left(\operatorname{ad} \mathfrak{n}^{-}\right) u_{\nu}=0$. Therefore $u_{\nu} \widetilde{M}(\mu)=0$. Since Ann $\widetilde{M}(\mu)$ is ad $\mathfrak{g}$-stable, $N \subset \operatorname{Ann} \widetilde{M}(\mu)$ and, in particular, $N \subset \operatorname{Ann} \widetilde{V}\left(s_{\alpha} \cdot \mu\right)$.

Hence $\sum_{j=0}^{s} p_{j}(\mu)\left(\Psi^{-1} \theta_{j}\right)(\widetilde{V})$ annihilates $\widetilde{V}\left(s_{\alpha} \cdot \mu\right)$ for any $\mu \in R$. Then, by Lemma 4.1 .1

$$
\left(\sum_{j=0}^{s}\left(s_{\alpha} \cdot p_{j}\right) \theta_{j}(v)\right)\left(\mu^{\prime}\right)=0
$$

for any $\left.v \in \widetilde{V}\right|_{0}$ and any $\mu^{\prime}$ such that $s_{\alpha} \cdot \mu^{\prime} \in R$. The terms $\sum_{j=0}^{s}\left(s_{\alpha} \cdot p_{j}\right) \theta_{j}(v)$ are polynomials in $\mathcal{S}(\mathfrak{h})$. Since $R$ is a Zariski dense subset of $\mathfrak{h}^{*}$, one concludes that 
these polynomials are equal to zero. Consequently

$$
\sum_{j=0}^{s}\left(s_{\alpha} \cdot p_{j}\right) \theta_{j}=0
$$

Taking into account that $\sum_{j=0}^{s} p_{j} \theta_{j}=0$ and that $\theta_{1}, \ldots, \theta_{s}$ are $\mathcal{S}(\mathfrak{h})$-linearly independent, one concludes that $p_{j} / p_{0}=\left(s_{\alpha} \cdot p_{j}\right) /\left(s_{\alpha} \cdot p_{0}\right)$ for all $j=1, \ldots, s$. Thus $p_{j} / p_{0}$ is $W$-invariant for all $j=1, \ldots, s$. Then, by Lemma 11.5, for each $j=0, \ldots, s$ there exists $q_{j} \in \mathcal{S}(\mathfrak{h})^{W}$. such that $q_{j} / q=p_{j} / p_{0}$. By 3.3.2 $t^{2} \mathcal{S}(\mathfrak{h})^{W} \subset$ $\mathcal{P}(\mathcal{Z}(\mathfrak{g}))$ and thus $t^{2} q_{j} \in \mathcal{P}(\mathcal{Z}(\mathfrak{g}))$ for all $j=0, \ldots, s$. Since $q_{j} / q=p_{j} / p_{0}$ one has

$$
\left(t^{2} q\right) \theta_{0}+\sum_{j=1}^{s}\left(t^{2} q_{j}\right) \theta_{j}=0
$$

This completes the proof.

4.4.3. Proposition. Let $\theta_{1}, \ldots, \theta_{s} \in \mathcal{I}(\widetilde{V})$ be an $\mathcal{S}(\mathfrak{h})$-basic system of $\mathcal{I}(\tilde{V}) \mathcal{S}(\mathfrak{h})$, let $p$ be a minor of this system, and let $z \in \mathcal{Z}(\mathfrak{g})$ be such that $\mathcal{P}(z)=t^{2} q$ where $q$ is a maximal $W$-invariant divisor of $p^{|W|}$. Then the localized module $\operatorname{Hom}_{\mathfrak{g}}\left(\widetilde{V}, \widetilde{\mathcal{U}}\left[z^{-1}\right]\right)$ is freely generated over $\mathcal{Z}(\mathfrak{g})\left[z^{-1}\right]$ by $\left\{\Psi^{-1} \theta_{1}, \ldots, \Psi^{-1} \theta_{s}\right\}$.

Proof. Recall that $\Psi$ provides a $\mathcal{Z}(\mathfrak{g})$-isomorphism from $\operatorname{Hom}_{\mathfrak{g}}(\widetilde{V}, \widetilde{\mathcal{U}})$ onto $\mathcal{I}(\widetilde{V})$. Therefore the required assertion is equivalent to the following statement: the localized module $\mathcal{I}(\widetilde{V}) \otimes_{\mathcal{Z}(\mathfrak{g})} \mathcal{Z}(\mathfrak{g})\left[z^{-1}\right]$ is freely generated over $\mathcal{Z}(\mathfrak{g})\left[z^{-1}\right]$ by $\left\{\theta_{1}, \ldots, \theta_{s}\right\}$. In other words, one has to show that for any $\theta_{0} \in \mathcal{I}(\widetilde{V})$ there exists a unique collection $z_{1}, \ldots, z_{s}$ such that $z \theta_{0}+\sum_{1}^{s} z_{j} \theta_{j}=0$ or, equivalently, that there exists a unique collection $a_{1}, \ldots, a_{s} \in \mathcal{P}(\mathcal{Z}(\mathfrak{g})) \subset \mathcal{S}(\mathfrak{h})$ such that

$$
\left(t^{2} q\right) \theta_{0}+\sum_{1}^{s} a_{j} \theta_{j}=0 .
$$

The uniqueness immediately follows from the fact that the collection $\theta_{1}, \ldots, \theta_{s}$ is an $\mathcal{S}(\mathfrak{h})$-basic system of $\mathcal{I}(\widetilde{V}) \mathcal{S}(\mathfrak{h})$. To prove the existence, recall that $\mathcal{I}(\widetilde{V}) \mathcal{S}(\mathfrak{h})$ is an $\mathcal{S}(\mathfrak{h})$-submodule of $\operatorname{Hom}\left(\left.\widetilde{V}\right|_{0}, \mathcal{S}(\mathfrak{h})\right)$. By 4.4.1 for any $\theta_{0} \in \mathcal{I}(\widetilde{V})$ there exists a unique collection $p_{1}, \ldots, p_{s} \in \mathcal{S}(\mathfrak{h})$ such that $p \theta_{0}+\sum_{j=1}^{s} p_{j} \theta_{j}=0$. Now the required existence follows from Lemma 4.4.2.

4.4.4. Corollary. The $\mathcal{Z}(\mathfrak{g})$-rank of $\operatorname{Hom}_{\mathfrak{g}}(\widetilde{V}, \widetilde{\mathcal{U}})$ is not greater than $\left.\operatorname{dim} \widetilde{V}\right|_{0}$.

4.4.5. Remark. Set $r=\left.\operatorname{dim} \tilde{V}\right|_{0}$. Assume that $\operatorname{Hom}_{\mathfrak{g}}(\widetilde{V}, \widetilde{\mathcal{U}})$ contains the collection $\theta_{1}, \ldots, \theta_{r}$ possessing the following property: for a certain $\lambda \in \mathfrak{h}^{*}$ the images of the modules $\theta_{1}(\widetilde{V}), \ldots, \theta_{r}(\widetilde{V})$ under the map $\widetilde{\mathcal{U}} \rightarrow \widetilde{\mathcal{U}} /$ Ann $\widetilde{V}(\lambda)$ form a direct sum. We claim that $\theta_{1}, \ldots, \theta_{r}$ is a $\mathcal{Z}(\mathfrak{g})$-basic system of $\operatorname{Hom}_{\mathfrak{g}}(\widetilde{V}, \widetilde{\mathcal{U}})$.

In fact, choose a basis $v_{1}, \ldots, v_{r}$ of $\left.\tilde{V}\right|_{0}$. By Corollary 4.1.2 the rank of the matrix $\left(\mathcal{P}\left(\theta_{j}\left(v_{i}\right)\right)(\lambda)\right)_{i=1, r}^{j=1, r}$ is equal to $r$. Hence the rank of the matrix

$$
\left(\Psi\left(\theta_{j}\left(v_{i}\right)\right)\right)_{i=1, r}^{j=1, r}=\left(\mathcal{P}\left(\theta_{j}\left(v_{i}\right)\right)\right)_{i=1, r}^{j=1, r} \in M a t_{(r \times r)} \mathcal{S}(\mathfrak{h})
$$

is also equal to $r$ and so $\Psi \theta_{1}, \ldots, \Psi \theta_{r}$ are $\mathcal{S}(\mathfrak{h})$-linearly independent in $\mathcal{I}(\widetilde{V}) \mathcal{S}(\mathfrak{h})$. Since $\mathcal{I}(\widetilde{V}) \mathcal{S}(\mathfrak{h})$ is an $\mathcal{S}(\mathfrak{h})$-submodule of $\operatorname{Hom}\left(\left.\widetilde{V}\right|_{0}, \widetilde{\mathcal{U}}\right)$, its rank is not greater than $r$. Hence $\Psi \theta_{1}, \ldots, \Psi \theta_{r}$ is an $\mathcal{S}(\mathfrak{h})$-basic system of $\mathcal{I}(\widetilde{V}) \mathcal{S}(\mathfrak{h})$. Therefore $\theta_{1}, \ldots, \theta_{r}$ is a $\mathcal{Z}(\mathfrak{g})$-basic system of $\operatorname{Hom}_{\mathfrak{g}}(\widetilde{V}, \widetilde{\mathcal{U}})$ by Proposition 4.4.3. 
4.5. In this subsection we show that the $\mathcal{Z}(\mathfrak{g})$-rank of $\operatorname{Hom}_{\mathfrak{g}}(\widetilde{V}, \widetilde{\mathcal{U}})$ is equal to $\left.\operatorname{dim} \tilde{V}\right|_{0}$.

4.5.1. Separation Theorem 2.8.1 claims the existence of an ad $\mathfrak{g}_{0}$-submodule $H$ of $\mathcal{U}$ such that the multiplication map provides an isomorphism $H \otimes \mathcal{Z}\left(\mathfrak{g}_{0}\right) \stackrel{\sim}{\longrightarrow} \mathcal{U}$. In both proofs ( $\mathrm{KO},[\mathrm{BL}]$ ) one constructs, actually, an ad $\mathfrak{g}_{0}$-submodule $H^{\prime}$ of the symmetric algebra $\mathcal{S}\left(\mathfrak{g}_{0}\right)$ such that the multiplication map provides an isomorphism $H^{\prime} \otimes \mathcal{S}\left(\mathfrak{g}_{0}\right)^{\mathfrak{g}_{0}} \stackrel{\sim}{\longrightarrow} \mathcal{S}\left(\mathfrak{g}_{0}\right)$.

Lemma. Let $H$ be an ad $\mathfrak{g}_{0}$-submodule of $\mathcal{U}$ such that the multiplication map provides an isomorphism gr $H \otimes \mathcal{S}\left(\mathfrak{g}_{0}\right)^{\mathfrak{g}_{0}} \rightarrow \mathcal{S}\left(\mathfrak{g}_{0}\right)$. Then the ad $\mathfrak{g}$-module $L:=$ $(\operatorname{ad} \widetilde{\mathcal{U}})(H T)$ is isomorphic to

$$
\bigoplus_{\widetilde{V} \in \operatorname{Irr}} \mathrm{E}(\widetilde{V})^{\left.\oplus \operatorname{dim} \tilde{V}\right|_{0}}
$$

and the multiplication induces the monomorphism $L \otimes \mathcal{Z}(\mathfrak{g}) \rightarrow \widetilde{\mathcal{U}}$.

Proof. Fix $H$ satisfying the above condition. According to $3.3, L:=(\operatorname{ad} \widetilde{\mathcal{U}})(H T)=$ $\left(\operatorname{ad}^{\prime} \widetilde{\mathcal{U}}\right)(H) T$ and as an ad $\mathfrak{g}$-module $L$ is isomorphic to the $\operatorname{ad}^{\prime} \mathfrak{g}$-module $\left(\operatorname{ad}^{\prime} \tilde{\mathcal{U}}\right)(H)$ $\cong \operatorname{Ind}_{\mathfrak{g}_{0}}^{\mathfrak{g}} H$. Now the required isomorphism follows from (3).

Since $L=\left(\operatorname{ad}^{\prime} \tilde{\mathcal{U}}\right)(H) T$ and $T$ is a non-zero divisor, the injectivity of the map $L \otimes \mathcal{Z}(\mathfrak{g}) \rightarrow \widetilde{\mathcal{U}}$ is equivalent to the injectivity of the map $\left(\operatorname{ad}^{\prime} \widetilde{\mathcal{U}}\right)(H) \otimes \mathcal{Z}(\mathfrak{g}) \rightarrow \widetilde{\mathcal{U}}$ (both maps are restrictions of the multiplication map). To check the injectivity of the map $\left(\operatorname{ad}^{\prime} \tilde{\mathcal{U}}\right)(H) \otimes \mathcal{Z}(\mathfrak{g}) \rightarrow \widetilde{\mathcal{U}}$ it is enough to check the injectivity of its "graded version":

$$
\operatorname{gr}\left(\left(\operatorname{ad}^{\prime} \tilde{\mathcal{U}}\right)(H)\right) \otimes \operatorname{gr} \mathcal{Z}(\mathfrak{g}) \rightarrow \mathcal{S}(\mathfrak{g}) .
$$

Recall that $\operatorname{gr} \mathcal{Z}(\mathfrak{g})=\mathcal{S}(\mathfrak{g})^{\mathfrak{g}}$. Let $\left\{x_{i}\right\}_{i \in I}$ be an ordered basis of $\mathfrak{g}_{1}$. For any $J \subseteq I$ set $x_{J}:=\prod_{j \in J} x_{j}$ where the product is taken with respect to the given order. Assume that

$$
\sum_{J \subseteq I ; k=1, \ldots, s} z_{k, J} \operatorname{gr}\left(\left(\operatorname{ad}^{\prime} x_{J}\right) h_{k}\right)=0
$$

for some non-zero $h_{1}, \ldots, h_{s} \in H$ and some $z_{k, J} \in \mathcal{S}(\mathfrak{g})^{\mathfrak{g}}$. To check the injectivity one has to show that all elements $z_{k, J}$ are equal to zero. Suppose not. For $x \in \mathfrak{g}_{1}, u \in \widetilde{\mathcal{U}}$ one has $\left(\operatorname{ad}^{\prime} x\right) u=2 x u-(\operatorname{ad} x) u$. This implies $\operatorname{gr}\left(\left(\operatorname{ad}^{\prime} x_{J}\right) u\right)=$ $2^{|J|} \operatorname{gr} x_{J}$ gr $u$ for any $J \subseteq I$ and $u \in \mathcal{U}$. For each $J \subseteq I$ denote by $P_{J}$ the projection $\mathcal{S}(\mathfrak{g}) \rightarrow \mathcal{S}\left(\mathfrak{g}_{0}\right) \operatorname{gr} x_{J}$ with the kernel $N_{J}:=\sum_{J^{\prime} \subseteq I, J^{\prime} \neq J} \mathcal{S}\left(\mathfrak{g}_{0}\right) \operatorname{gr} x_{J^{\prime}}$. By 2.3 .2 the restriction of $P_{\emptyset}$ to $\mathcal{S}(\mathfrak{g})^{\mathfrak{g}}$ provides a monomorphism $\mathcal{S}(\mathfrak{g})^{\mathfrak{g}} \rightarrow \mathcal{S}\left(\mathfrak{g}_{0}\right)^{\mathfrak{g}_{0}}$. Choose $J \subseteq I$ such that $z_{k, J} \neq 0$ for some $k$ and $J$ is a minimal (under inclusion) subset of $I$ possessing this property. Then

$$
\begin{aligned}
0 & =P_{J}\left(\sum_{J^{\prime} \subseteq I, k=1, \ldots, s} z_{k, J^{\prime}} \operatorname{gr}\left(\left(\operatorname{ad}^{\prime} x_{J^{\prime}}\right) h_{k}\right)\right) \\
& \left.=P_{J}\left(\sum_{J^{\prime} \subseteq I, k=1, \ldots, s} 2^{\left|J^{\prime}\right|} z_{k, J^{\prime}} \operatorname{gr} x_{J^{\prime}} \operatorname{gr} h_{k}\right)\right) \\
& =2^{|J|} \operatorname{gr} x_{J} \sum_{k=1, \ldots, s} P_{\emptyset}\left(z_{k, J}\right) \operatorname{gr} h_{k} .
\end{aligned}
$$


Since gr $H \otimes \mathcal{S}\left(\mathfrak{g}_{0}\right)^{\mathfrak{g}_{0}} \stackrel{\sim}{\longrightarrow} \mathcal{S}\left(\mathfrak{g}_{0}\right)$, all elements $z_{k, J}$ are equal to zero. The lemma is proved.

4.5.2. Corollary. The $\mathcal{Z}(\mathfrak{g})$-rank of $\operatorname{Hom}_{\mathfrak{g}}(\widetilde{V}, \widetilde{\mathcal{U}})$ is equal to $\left.\operatorname{dim} \widetilde{V}\right|_{0}$.

Proof. Fix $\widetilde{V} \in \operatorname{Irr}$ and set $r:=\left.\operatorname{dim} \widetilde{V}\right|_{0}$. Choose $H$ satisfying the assumption of Lemma 4.5.1. Then the vector space $\operatorname{Hom}_{\mathfrak{g}}(\widetilde{V}, L)$ has dimension $r$; let $\theta_{1}, \ldots, \theta_{r}$ be a basis of $\operatorname{Hom}_{\mathfrak{g}}(\widetilde{V}, L)$. The injectivity of the map $L \otimes \mathcal{Z}(\mathfrak{g}) \rightarrow \widetilde{\mathcal{U}}$, induced by the multiplication, implies the $\mathcal{Z}(\mathfrak{g})$-linear independence of $\theta_{1}, \ldots, \theta_{r}$ in $\operatorname{Hom}_{\mathfrak{g}}(\widetilde{V}, \widetilde{\mathcal{U}})$. Thus the $\mathcal{Z}(\mathfrak{g})$-rank of $\operatorname{Hom}_{\mathfrak{g}}(\widetilde{V}, \widetilde{\mathcal{U}})$ is greater than or equal to $r$. Comparing with Corollary 4.4.4, one concludes the required assertion.

\subsection{PRV determinants.}

4.6.1. Definitions. Fix $\widetilde{V} \in \operatorname{Irr}$ and set $r:=\left.\operatorname{dim} \widetilde{V}\right|_{0}$. Lemma 4.4 .2 (i) implies that $\theta_{1}, \ldots, \theta_{r}$ is a $\mathcal{Z}(\mathfrak{g})$-basic system of $\operatorname{Hom}_{\mathfrak{g}}(\widetilde{V}, \widetilde{\mathcal{U}})$ iff $\Psi \theta_{1}, \ldots, \Psi \theta_{r}$ is an $\mathcal{S}(\mathfrak{h})$-basic system of $\mathcal{I}(\widetilde{V}) \mathcal{S}(\mathfrak{h})$. By 4.4.1, the last is equivalent to the condition that $\operatorname{det} C \neq 0$ where

$$
C:=\left(\mathcal{P}\left(\theta_{j}\left(v_{i}\right)\right)\right) \in \operatorname{Mat}_{(r \times r)}(\mathcal{S}(\mathfrak{h}))
$$

and $\left\{v_{1}, \ldots, v_{r}\right\}$ is a basis of $\left.\widetilde{V}\right|_{0}$.

Call such a matrix a PRV matrix (corresponding to the basic system $\theta_{1}, \ldots, \theta_{r}$ ) and the determinant $\operatorname{det} C$ a $P R V$ determinant. Note that for different choices of a basis $\left\{v_{1}, \ldots, v_{r}\right\}$ of $\left.\widetilde{V}\right|_{0}$ the PRV matrix corresponding to $\theta_{1}, \ldots, \theta_{r}$ differs by the multiplication on an invertible scalar matrix.

Denote by $\mathcal{J}(\widetilde{V})$ the set of all PRV matrices and by $\operatorname{det} \mathcal{J}(\widetilde{V})$ the set of PRV determinants (for fixed $\widetilde{V} \in \operatorname{Irr}$ ). One has

$$
\operatorname{det} \mathcal{J}(\widetilde{V})=\left\{\operatorname{det}\left(\mathcal{P}\left(\theta_{j}\left(v_{i}\right)\right)\right) \mid \theta_{1}, \ldots, \theta_{r} \in \operatorname{Hom}_{\mathfrak{g}}(\widetilde{V}, \widetilde{\mathcal{U}})\right\} \backslash\{0\} .
$$

For each $\nu \in \mathfrak{h}^{*}$ such that $\operatorname{dim} \widetilde{V}(\nu)<\infty \operatorname{set} \operatorname{det} \mathcal{J}(\nu):=\operatorname{det} \mathcal{J}(\widetilde{V}(\nu))$. For $\lambda \in \mathfrak{h}^{*}$ we write $\operatorname{det} \mathcal{J}(\widetilde{V})(\lambda)=0$ if $(\operatorname{det} C)(\lambda)=0$ for all $C \in \mathcal{J}(\widetilde{V})$.

If $\theta_{1}, \ldots, \theta_{r}$ is a $\mathcal{Z}(\mathfrak{g})$-basic system of $\operatorname{Hom}_{\mathfrak{g}}(\widetilde{V}, \widetilde{\mathcal{U}})$, then for any non-zero $z \in$ $\mathcal{Z}(\mathfrak{g})$ the collection $z \theta_{1}, \theta_{2}, \ldots, \theta_{r}$ is also a $\mathcal{Z}(\mathfrak{g})$-basic system. Consequently, $\operatorname{det} \mathcal{J}(\widetilde{V})$ is closed under the multiplication on the non-zero elements of $\mathcal{P}(\mathcal{Z}(\mathfrak{g}))$.

4.6.2. Let $N$ be an ad $\mathfrak{g}$-submodule of $\widetilde{\mathcal{U}}$ such that $\operatorname{dim} \operatorname{Hom}_{\mathfrak{g}}(\widetilde{V}, N)=\left.\operatorname{dim} \widetilde{V}\right|_{0}$. By a slight abuse of notation, we shall denote by $\operatorname{det} \mathcal{J}(\widetilde{V} ; N)$ the determinant of the matrix $\left(\mathcal{P}\left(\theta_{j}\left(v_{i}\right)\right)\right)$ where $\theta_{1}, \ldots, \theta_{r}\left(\operatorname{resp}, v_{1}, \ldots, v_{r}\right)$ is a basis of $\operatorname{Hom}_{\mathfrak{g}}(\widetilde{V}, N)$ $\left(\right.$ resp., $\left.\left.\widetilde{V}\right|_{0}\right)$. Note that $\operatorname{det} \mathcal{J}(\widetilde{V} ; N)$ can be zero.

For different choices of a basis $\theta_{1}, \ldots, \theta_{r}$, the values of $\operatorname{det} \mathcal{J}(\widetilde{V} ; N)$ differ by a multiplicative constant. Hence $\operatorname{det} \mathcal{J}(\widetilde{V} ; N)$ is a polynomial in $\mathcal{S}(\mathfrak{h})$ defined up to a non-zero scalar.

4.6.3. Remark. The original definition of PRV determinants given in [PRV] for the semisimple Lie algebras differs from our definition. Namely, the PRV determinant $p(V)$ is equal to the polynomial $\operatorname{det} \mathcal{J}(V ; H)$ where $H$ is a harmonic space (see 2.8.1). This definition works for completely reducible Lie superalgebras as well. In our notation $\operatorname{det} \mathcal{J}(V)=\mathcal{S}(\mathfrak{h})^{W \cdot} \cdot p(V) \backslash\{0\}$ — this follows from Separation Theorem 2.8.1. 
4.6.4. Take $\widetilde{V} \in \operatorname{Irr}$ and $p \in \operatorname{det} \mathcal{J}(\widetilde{V})$. Let $\theta_{1}, \ldots, \theta_{r}$ be a $\mathcal{Z}(\mathfrak{g})$-basic system of $\operatorname{Hom}_{\mathfrak{g}}(\widetilde{V}, \widetilde{\mathcal{U}})$ such that the determinant of the corresponding PRV matrix is equal to $p$. Denote by $q$ the maximal $W$.-invariant divisor of $p$. Combining Remark 4.4.5 and Proposition 4.4.3. one concludes that $z \in \mathcal{Z}(\mathfrak{g})$ satisfying $\mathcal{P}(z)=t^{2} q^{|W|}$ possesses the following property: for any $\theta \in \operatorname{Hom}_{\mathfrak{g}}(\widetilde{V}, \widetilde{\mathcal{U}})$ one has $z \theta=\sum_{i=1}^{r} z_{i} \theta_{i}$ for certain $z_{i} \in \mathcal{Z}(\mathfrak{g})$.

In particular, for $a:=\mathcal{P}(z)^{r}=t^{2 r} q^{r|W|}$ one has

$$
a \cdot \operatorname{det} \mathcal{J}(\widetilde{V}) \subset \mathcal{P}(\mathcal{Z}(\mathfrak{g})) p \subseteq \mathcal{S}(\mathfrak{h})^{W \cdot} p
$$

On the other hand, $\mathcal{P}(\mathcal{Z}(\mathfrak{g})) p \subseteq \operatorname{det} \mathcal{J}(\widetilde{V}) \cup\{0\}$ by 4.6.1.

4.6.5. Corollary. Take $\widetilde{V} \in \operatorname{Irr}$. For any $p \in \operatorname{det} \mathcal{J}(\widetilde{V})$ there exists $a \in \mathcal{S}(\mathfrak{h})^{W}$. such that

$$
\mathcal{P}(\mathcal{Z}(\mathfrak{g}) \backslash\{0\}) p \subseteq \operatorname{det} \mathcal{J}(\widetilde{V}) \subset \mathcal{S}(\mathfrak{h})^{W \cdot}\left[a^{-1}\right] p .
$$

4.6.6. Fix $\tilde{V} \in$ Irr. Take a basic system $\theta_{1}, \ldots, \theta_{r}$ and denote by $C$ the corresponding PRV matrix. Recall that $\operatorname{det} C \neq 0$. For each $\lambda \in \mathfrak{h}^{*}$ denote by $C(\lambda)$ the complex matrix which is obtained from $C$ by the evaluation of all entries at $\lambda$. Clearly $\operatorname{det} C(\lambda)=(\operatorname{det} C)(\lambda)$. By Corollary 4.1.2,

$$
\operatorname{corank} C(\lambda)=\operatorname{dim} \operatorname{Hom}_{\mathfrak{g}}\left(\widetilde{V}, \operatorname{Ann} \widetilde{V}(\lambda) \cap \sum_{j=1}^{r} \theta_{j}(\widetilde{V})\right) .
$$

In particular, Ann $\widetilde{V}(\lambda) \cap \sum_{j=1}^{r} \theta_{j}(\widetilde{V})=0$ iff $\operatorname{det} C(\lambda) \neq 0$. Then, setting $f_{\lambda}$ to be the natural map $\widetilde{\mathcal{U}} \rightarrow \operatorname{End}(\widetilde{V}(\lambda))$, one has

$$
\operatorname{dim} \operatorname{Hom}_{\mathfrak{g}}\left(\widetilde{V}, f_{\lambda}(\operatorname{Soc} \widetilde{\mathcal{U}})\right)=\max \{\operatorname{rank} C(\lambda) \mid C \in \mathcal{J}(\widetilde{V})\} .
$$

Combining 11.4.1 and (4) one obtains

4.6.7. Corollary. Let $\widetilde{M}(\lambda)$ be simple. Then the image of the socle of $\widetilde{\mathcal{U}}$ under the natural map $\widetilde{\mathcal{U}} \rightarrow F(\widetilde{M}(\lambda), \widetilde{M}(\lambda))$ coincides with the socle of $F(\widetilde{M}(\lambda), \widetilde{M}(\lambda))$ iff $\operatorname{det} \mathcal{J}(\widetilde{V})(\lambda) \neq 0$ for any $\widetilde{V} \in \operatorname{Irr}$.

4.6.8. Change of Borel. In the definition of PRV matrices we use the HarishChandra projection. Therefore this definition depends on the choice of triangular decomposition. In the sequel we will add a lower index to designate the corresponding Borel subsuperalgebra in the case when the choice of triangular decomposition is not clear from the context. For instance, $\mathcal{J}_{\mathfrak{b}}(\widetilde{V})$ is the set of PRV matrices of the form $\left(\mathcal{P}_{\mathfrak{b}}\left(\theta_{j}\left(v_{i}\right)\right)\right)$ where $\theta_{1}, \ldots, \theta_{r}$ is a $\mathcal{Z}(\mathfrak{g})$-basic system of $\operatorname{Hom}_{\mathfrak{g}}(\widetilde{V}, \widetilde{\mathcal{U}})$ and $v_{1}, \ldots, v_{r}$ is a basis of $\left.\widetilde{V}\right|_{0}$.

Let $\mathfrak{b}$ and $\mathfrak{b}^{\prime}$ be connected by the odd reflection along an odd isotropic root $\beta$. By 2.7, $\widetilde{V}_{\mathfrak{b}}(\lambda)=\widetilde{V}_{\mathfrak{b}^{\prime}}\left(\lambda^{\prime}\right)$ where $\lambda=\lambda^{\prime}$ if $\lambda$ is such that $(\lambda, \beta)=0$, and $\lambda=\lambda^{\prime}+\beta$ otherwise. Then for any $\widetilde{V} \in \operatorname{Irr}$ equality (4) implies

$$
\begin{gathered}
\max \left\{\operatorname{rank} C(\lambda) \mid C \in \mathcal{J}_{\mathfrak{b}_{1}}(\widetilde{V})\right\}=\max \left\{\operatorname{rank} C\left(\lambda^{\prime}\right) \mid C \in \mathcal{J}_{\mathfrak{b}_{2}}(\widetilde{V})\right\}, \\
\operatorname{det} \mathcal{J}_{\mathfrak{b}_{1}}(\widetilde{V})(\lambda)=0 \Longleftrightarrow \operatorname{det} \mathcal{J}_{\mathfrak{b}_{2}}(\widetilde{V})\left(\lambda^{\prime}\right)=0 .
\end{gathered}
$$

4.6.9. Remark. Equality (4) has the following consequence: for any PRV determinant $p \in \operatorname{det} \mathcal{J}(\widetilde{V})$ and any $\lambda \in \mathfrak{h}^{*}$, the order of zero of $p$ at the point $\lambda$ is greater than or equal to the value $\left(\left.\operatorname{dim} \widetilde{V}\right|_{0}-\operatorname{dim} \operatorname{Hom}_{\mathfrak{g}}(\widetilde{V}, \operatorname{End}(\widetilde{V}(\lambda)))\right)$. 
In the case when $\mathfrak{g}$ is completely reducible, this property is used for the calculation of PRV determinants - see [JL], [J3], GL1]. Recall that, in this case, the PRV determinant is a polynomial defined up to a non-zero scalar (see Remark 4.6.3).

\subsection{Separation type theorem.}

4.7.1. Definition. Call an ad $\mathfrak{g}$-submodule $L$ of $\widetilde{\mathcal{U}}$ a generic harmonic space if the multiplication map provides an isomorphism $L \otimes \mathcal{Z}(\mathfrak{g})\left[S^{-1}\right] \stackrel{\sim}{\longrightarrow} \widetilde{\mathcal{U}}\left[S^{-1}\right]$ for certain $S \subset(\mathcal{Z}(\mathfrak{g}) \backslash\{0\})$.

In Theorem 4.7.4 we describe all generic harmonic spaces in $\widetilde{\mathcal{U}}$. Of course, if $L$ is a generic harmonic space, then $L \otimes \mathcal{Z}(\mathfrak{g})\left[S^{-1}\right] \stackrel{\sim}{\sim} \widetilde{\mathcal{U}}\left[S^{-1}\right]$ for $S:=\mathcal{Z}(\mathfrak{g}) \backslash\{0\}$. However it is always possible to find a smaller $S$; in Corollary 4.7 .7 we describe such $S$ in terms of PRV determinants. As shown in Section 7 for $\mathfrak{g}=\mathfrak{s l}(m, n)$ or $\mathfrak{o} \mathfrak{s p}(2,2 n)$ there is a generic harmonic space in $\widetilde{\mathcal{U}}$ such that one can take $S=\left\{T^{2}\right\}$.

4.7.2. Lemma. If an ad $\mathfrak{g}$-submodule $L$ of $\widetilde{\mathcal{U}}$ is such that

$$
\begin{aligned}
& \text { (a) } \quad L \cong \bigoplus \bigoplus_{\widetilde{V} \in \operatorname{Irr}} \mathrm{E}(\widetilde{V})^{\left.\oplus \operatorname{dim} \tilde{V}\right|_{0},} \\
& \text { (b) } \quad \forall \widetilde{V} \in \operatorname{Irr} \quad \operatorname{det} \mathcal{J}(\widetilde{V} ; L) \neq 0,
\end{aligned}
$$

then it is a generic harmonic space.

Proof. Set $\mathfrak{h}_{f}^{*}:=\{\nu \mid \operatorname{dim} \widetilde{V}(\nu)<\infty\}$. For each $\nu \in \mathfrak{h}_{f}^{*}$ choose a basis $\theta_{1}^{\nu}, \ldots, \theta_{r(\nu)}^{\nu}$ of $\operatorname{Hom}_{\mathfrak{g}}(\tilde{V}(\nu), L)$. Recall that $r(\nu)=\left.\operatorname{dim} \tilde{V}(\nu)\right|_{0}$. By 4.6.1, the collection $\theta_{1}^{\nu}, \ldots$, $\theta_{r(\nu)}^{\nu}$ forms a $\mathcal{Z}(\mathfrak{g})$-basic system of $\operatorname{Hom}_{\mathfrak{g}}(\widetilde{V}(\nu), \widetilde{\mathcal{U}})$. By 4.6.4, for a suitable $z(\nu) \in$ $\mathcal{Z}(\mathfrak{g})$ the collection $\theta_{1}^{\nu}, \ldots, \theta_{r(\nu)}^{\nu}$ forms a free $\mathcal{Z}(\mathfrak{g})\left[z(\nu)^{-1}\right]$-basis of the localized module $\operatorname{Hom}_{\mathfrak{g}}\left(\widetilde{V}(\nu), \widetilde{\mathcal{U}}\left[z(\nu)^{-1}\right]\right)$.

Set $S:=\left\{z(\nu), \nu \in \mathfrak{h}_{f}^{*}\right\}$. Denote by $\psi$ the map $L \otimes \mathcal{Z}(\mathfrak{g})\left[S^{-1}\right] \rightarrow \widetilde{\mathcal{U}}\left[S^{-1}\right]$ induced by the multiplication. For any $\nu \in \mathfrak{h}_{f}^{*}$ the collection $\theta_{1}^{\nu}, \ldots, \theta_{r(\nu)}^{\nu}$ forms a free $\mathcal{Z}(\mathfrak{g})\left[S^{-1}\right]$-basis of the localized module $\operatorname{Hom}_{\mathfrak{g}}\left(\widetilde{V}(\nu), \widetilde{\mathcal{U}}\left[S^{-1}\right]\right)$. This means that the restriction of $\phi$ on the space $\sum_{i=1}^{s(\nu)} \theta_{i}^{\nu}(\widetilde{V}) \otimes \mathcal{Z}(\mathfrak{g})\left[S^{-1}\right]$ is a monomorphism and its image coincides with the isotypical component of $\widetilde{V}(\nu)$ in the socle of $\widetilde{\mathcal{U}}\left[S^{-1}\right]$. Then the restriction of $\phi$ on Soc $L \otimes \mathcal{Z}(\mathfrak{g})\left[S^{-1}\right]$ is a monomorphism and $\operatorname{Soc} \widetilde{\mathcal{U}}\left[S^{-1}\right]=$ $\phi\left(\operatorname{Soc} L \otimes \mathcal{Z}(\mathfrak{g})\left[S^{-1}\right]\right)$. Using the equality $\operatorname{Soc} L \otimes \mathcal{Z}(\mathfrak{g})\left[S^{-1}\right]=\operatorname{Soc}\left(L \otimes \mathcal{Z}(\mathfrak{g})\left[S^{-1}\right]\right)$ and 3.1 .2 one concludes that $\phi$ is a monomorphism. From 3.2.3, it follows that $L \otimes$ $\mathcal{Z}(\mathfrak{g})\left[S^{-1}\right]$ is an injective module in $\mathcal{F}$ in. Since the image of $\psi$ contains $\operatorname{Soc} \widetilde{\mathcal{U}}\left[S^{-1}\right]$, $\psi$ is a bijection. The lemma is proved.

4.7.3. Corollary. If $H$ satisfies the assumption of Lemma 4.5.1, then $(\operatorname{ad} \widetilde{\mathcal{U}})(H T)$ is a generic harmonic space.

Proof. By Lemma 4.5.1 $L:=(\operatorname{ad} \widetilde{\mathcal{U}})(H T)$ fulfills condition (a) of Lemma 4.7.2.

Fix $\widetilde{V} \in \operatorname{Irr}$ and choose a basis $\theta_{1}, \ldots, \theta_{r}$ of $\operatorname{Hom}_{\mathfrak{g}}(\widetilde{V}, L)$. Observe that $r=$ $\left.\operatorname{dim} \tilde{V}\right|_{0}$. The injectivity of the map $L \otimes \mathcal{Z}(\mathfrak{g}) \rightarrow \widetilde{\mathcal{U}}$, induced by the multiplication, implies the $\mathcal{Z}(\mathfrak{g})$-linear independence of $\theta_{1}, \ldots, \theta_{r}$ in $\operatorname{Hom}_{\mathfrak{g}}(\widetilde{V}, \widetilde{\mathcal{U}})$. Since the $\mathcal{Z}(\mathfrak{g})$ rank of the latter is equal to $r$, the collection $\theta_{1}, \ldots, \theta_{r}$ forms a $\mathcal{Z}(\mathfrak{g})$-basic system of $\operatorname{Hom}_{\mathfrak{g}}(\widetilde{V}, \widetilde{\mathcal{U}})$. Then, by 4.6.1, $\operatorname{det} \mathcal{J}(\widetilde{V} ; L) \neq 0$. By Lemma 4.7.2 $(\operatorname{ad} \widetilde{\mathcal{U}})(H T)$ is a generic harmonic space. 
4.7.4. Theorem. An ad $\mathfrak{g}$-submodule $L$ of $\widetilde{\mathcal{U}}$ is a generic harmonic space iff

(a) $L \cong \bigoplus_{\tilde{V} \in \operatorname{Irr}} \mathrm{E}(\tilde{V})^{\left.\oplus \operatorname{dim} \tilde{V}\right|_{0}}$

and one of the following conditions holds:

(b) $\forall \widetilde{V} \in \operatorname{Irr} \operatorname{det} \mathcal{J}(\widetilde{V} ; L) \neq 0$,

(c) the multiplication map provides an embedding $L \otimes \mathcal{Z}(\mathfrak{g}) \rightarrow \widetilde{\mathcal{U}}$.

Proof. By Lemma 4.7.2 $L$ satisfying (a) and (b) is a generic harmonic space. Arguing as in Corollary 4.7.3 one concludes that $L$ satisfying (a) and (c) is also a generic harmonic space.

It remains to show that any generic harmonic space $L$ fulfills conditions (a)-(c). Condition (c) obviously holds. Moreover, for any $\widetilde{V} \in \operatorname{Irr}$ a basis $\theta_{1}, \ldots, \theta_{r}$ of $\operatorname{Hom}_{\mathfrak{g}}(\tilde{V}, L)$ is a $\mathcal{Z}(\mathfrak{g})$-basic system of $\operatorname{Hom}_{\mathfrak{g}}(\widetilde{V}, \widetilde{\mathcal{U}})$. Therefore $\operatorname{det} \mathcal{J}(\widetilde{V} ; L) \neq 0$ and so (b) holds as well.

To verify condition (a), let us show that all generic harmonic spaces are pairwise isomorphic as ad $\mathfrak{g}$-modules. Indeed, let $L$ and $L^{\prime}$ be generic harmonic spaces. Since $\widetilde{\mathcal{U}}$ is countably dimensional, one can choose the corresponding sets $S, S^{\prime} \subset \mathcal{Z}(\mathfrak{g}) \backslash\{0\}$ having a countable number of elements. Take a maximal ideal $m$ of $\mathcal{Z}(\mathfrak{g})$ such that $m \cap\left(S \cup S^{\prime}\right)=\emptyset$. Then as ad $\mathfrak{g}$-modules $L \cong \tilde{\mathcal{U}} /(m \tilde{\mathcal{U}}) \cong L^{\prime}$. In Corollary 4.7.3 we construct a generic harmonic space satisfying (a). Hence all generic harmonic spaces satisfy condition (a). The theorem is proved.

4.7.5. Now we can formulate the following "receipt". For each $\widetilde{V} \in \operatorname{Irr}$ fix a $\mathcal{Z}(\mathfrak{g})$ basic system $\theta_{1}^{\widetilde{V}}, \ldots, \theta_{r}^{\widetilde{V}}$ of $\operatorname{Hom}_{\mathfrak{g}}(\widetilde{V}, \widetilde{\mathcal{U}})$. The module $\sum_{\widetilde{V} \in \operatorname{Irr} ; j} T^{2} \theta_{j}^{\widetilde{V}}(\widetilde{V})$ has an injective envelope inside an injective ad $\mathfrak{g}$-module $\tilde{\mathcal{U}} T$. This injective envelope is a generic harmonic space by Theorem 4.7.4.

4.7.6. Combining 4.6.4 and the proof of Lemma 4.7.2, one obtains

4.7.7. Corollary. Let $L$ be a generic harmonic space, and let $S \subset \mathcal{Z}(\mathfrak{g}) \backslash\{0\}$ satisfy the following property: for any $\widetilde{V} \in \operatorname{Irr}$ there exists $s \in S$ such that $\mathcal{P}(s)=t^{2} q$ where $q$ is a maximal $W$.-invariant divisor of $(\operatorname{det} \mathcal{J}(\widetilde{V} ; L))^{|W|}$. Then the multiplication map provides an isomorphism $L \otimes \mathcal{Z}(\mathfrak{g})\left[S^{-1}\right] \stackrel{\sim}{\longrightarrow} \widetilde{\mathcal{U}}\left[S^{-1}\right]$.

\section{Application to the description of Verma module annihilators}

In this section we prove Theorem 5.2 which provides a connection between PRV determinants and the annihilators of simple modules.

5.1. Proposition. For a simple strongly typical Verma module $\widetilde{M}(\lambda)$ the following conditions are equivalent:

(i) For any $\widetilde{V} \in \operatorname{Irr}$ one has $\operatorname{det} \mathcal{J}(\widetilde{V})(\lambda) \neq 0$.

(ii) The natural map $\widetilde{\mathcal{U}} \rightarrow F(\widetilde{M}(\lambda), \widetilde{M}(\lambda))$ is surjective and

$$
F(\widetilde{M}(\lambda), \widetilde{M}(\lambda)) \cong \bigoplus_{\widetilde{V} \in \operatorname{Irr}} \mathrm{E}(\widetilde{V})^{\left.\oplus \operatorname{dim} \tilde{V}\right|_{0}}
$$

Proof. Fix a strongly typical $\lambda$ such that $\widetilde{M}(\lambda)$ is simple. Denote by $f$ the natural $\operatorname{map} \widetilde{\mathcal{U}} \rightarrow F(\widetilde{M}(\lambda), \widetilde{M}(\lambda))$. 
Let us show that (ii) implies (i). Indeed, if both conditions of (ii) hold, then $F(\widetilde{M}(\lambda), \widetilde{M}(\lambda))$ is projective in $\mathcal{F}$ in and so $f$ has a left inverse $f^{-1}$. Denote by $N$ the image of $f^{-1}$. Take $\widetilde{V} \in \operatorname{Irr}$ and choose a basis $\theta_{1}, \ldots, \theta_{r}$ of $\operatorname{Hom}_{\mathfrak{g}}(\widetilde{V}, N)$. By Remark 4.4.5, $\theta_{1}, \ldots, \theta_{r}$ is a $\mathcal{Z}(\mathfrak{g})$-basic system of $\operatorname{Hom}_{\mathfrak{g}}(\widetilde{V}, \widetilde{\mathcal{U}})$. Denote by $C$ the corresponding PRV matrix. Since the restriction of $f$ to $N$ is a monomorphism corank $C(\lambda)=0$, by 4.6.6. Hence $\operatorname{det} C(\lambda) \neq 0$ and so $\operatorname{det} \mathcal{J}(\widetilde{V})(\lambda) \neq 0$ as required.

Let us show that (i) implies (ii). Suppose that (i) holds. By Corollary 4.6.7 $f(\operatorname{Soc} \widetilde{\mathcal{U}})$ coincides with the socle of $F(\widetilde{M}(\lambda), \widetilde{M}(\lambda))$. The socle of $\widetilde{\mathcal{U}}$ is a completely reducible $\mathfrak{g}$-module and so it contains a submodule $L$ such that the restriction of $f$ gives an isomorphism $L \stackrel{\sim}{\longrightarrow} \operatorname{Soc} F(\widetilde{M}(\lambda), \widetilde{M}(\lambda))$. The central element $T^{2}$ acts on $\widetilde{M}(\lambda)$ by a non-zero scalar $t(\lambda)^{2}$ (because $\lambda$ is strongly typical) and thus one can choose $L$ lying in $T^{2} \widetilde{\mathcal{U}}$. By 3.3 .2 , T $\widetilde{\mathcal{U}}$ is an injective ad $\mathfrak{g}$-submodule of $\widetilde{\mathcal{U}}$ and so it contains an injective envelope $\mathrm{E}(L)$ of $L$. The restriction of $f$ to $\mathrm{E}(L)$ is a monomorphism because the restriction of $f$ to $L$ is a monomorphism. Therefore $f(\mathrm{E}(L))$ is an injective module containing the socle of $F(\widetilde{M}(\lambda), \widetilde{M}(\lambda))$. Using 3.1.1, one concludes that the restriction of $f$ provides an isomorphism $\mathrm{E}(L) \stackrel{\sim}{\longrightarrow} F(\widetilde{M}(\lambda), \widetilde{M}(\lambda))$. In particular, $f$ is surjective and the ad $\mathfrak{g}$-module $F(\widetilde{M}(\lambda), \widetilde{M}(\lambda))$ is injective in $\mathcal{F}$ in. Combining these facts and 11.4.1 one obtains (ii).

5.2. Theorem. Suppose that $\lambda \in \mathfrak{h}^{*}$ is strongly typical and $\operatorname{det} \mathcal{J}(\widetilde{V})(\lambda) \neq 0$ for all $\widetilde{V} \in \operatorname{Irr}$. Then Ann $\widetilde{V}(\lambda)=\widetilde{\mathcal{U}} \operatorname{Ann}_{\mathcal{Z}(\mathfrak{g})} \widetilde{V}(\lambda)$.

Proof. For each $\tilde{V}(\nu) \in \operatorname{Irr}$ choose $p_{\nu} \in \operatorname{det} \mathcal{J}(\nu)$ such that $p_{\nu}(\lambda) \neq 0$. Let $\theta_{1}^{\nu}, \ldots, \theta_{r(\nu)}^{\nu} \in \operatorname{Hom}_{\mathfrak{g}}(\widetilde{V}(\nu), \widetilde{\mathcal{U}})$ be a $\mathcal{Z}(\mathfrak{g})$-basic system such that the determinant of the PRV matrix corresponding to this system is equal to $p_{\nu}$. Let $q_{\nu}$ be the maximal $W$.-invariant divisor of $p_{\nu}^{|W|}$, and let $z_{\nu} \in \mathcal{Z}(\mathfrak{g})$ be such that $\mathcal{P}\left(z_{\nu}\right)=t^{2} q_{\nu}$. Note that $z_{\nu} \notin \operatorname{Ann}_{\mathcal{Z}(\mathfrak{g})} \tilde{V}(\lambda)$ since $\left(t^{2} p_{\nu}\right)(\lambda) \neq 0$.

Let $S$ be the multiplicative closure of the set $\left\{T^{2} ; z_{\nu} \mid \widetilde{V}(\nu) \in \operatorname{Irr}\right\}$. Denote by $A$ the localization of $\mathcal{Z}(\mathfrak{g})$ by $S$ and by $\widetilde{\mathcal{U}}_{A}$ the localization of $\widetilde{\mathcal{U}}$ by $S$. The action of $\widetilde{\mathcal{U}}$ on $\widetilde{V}(\lambda)$ can be canonically extended to the action of the localized algebra $\widetilde{\mathcal{U}}_{A}$ since $S \cap \operatorname{Ann}_{\mathcal{Z}(\mathfrak{g})} \widetilde{V}(\lambda)=\emptyset$. Clearly, the ideal $\operatorname{Ann}_{A} \widetilde{V}(\lambda)$ is maximal in $A$.

Let us show that $\operatorname{Ann}_{\tilde{\mathcal{U}}_{A}} \tilde{V}(\lambda)=\widetilde{\mathcal{U}}_{A} \operatorname{Ann}_{A} \tilde{V}(\lambda)$. Combining 4.7.5 and Corollary 4.7.7, one concludes the existence of an ad $\mathfrak{g}$-submodule $H$ of $\widetilde{\mathcal{U}}$ such that

$$
\operatorname{Soc} H=\sum_{\nu: \tilde{V}(\nu) \in \operatorname{Irr}} \sum_{i=1}^{r(\nu)} T^{2} \operatorname{Im} \theta_{\nu}^{i}
$$

and that the multiplication map induces an isomorphism $H \otimes A \stackrel{\sim}{\longrightarrow} \widetilde{\mathcal{U}}_{A}$. To verify $\operatorname{Ann}_{\tilde{\mathcal{U}}_{A}} \tilde{V}(\lambda)=\widetilde{\mathcal{U}}_{A} \operatorname{Ann}_{A} \widetilde{V}(\lambda)$ it is enough to check that $H \cap \operatorname{Ann} \widetilde{V}(\lambda)=0$. Observe that $\operatorname{det} \mathcal{J}(\widetilde{V}(\nu) ; H)=p_{\nu} t^{2 r(\nu)}$ and so $\operatorname{det} \mathcal{J}(\widetilde{V}(\nu), H)(\lambda) \neq 0$ for all $\widetilde{V}(\nu) \in \operatorname{Irr}$. Then, by 4.6.6

$$
\left(\sum_{i=1}^{r(\nu)} \operatorname{Im} \theta_{\nu}^{i}\right) \cap \operatorname{Ann} \widetilde{V}(\lambda)=0,
$$

that is, Soc $H \cap \operatorname{Ann} \widetilde{V}(\lambda)=0$. Therefore $H \cap \operatorname{Ann} \widetilde{V}(\lambda)=0$ as required. Hence $\operatorname{Ann}_{\widetilde{\mathcal{U}}_{A}} \widetilde{M}(\lambda)=\widetilde{\mathcal{U}}_{A} \operatorname{Ann}_{A} \widetilde{M}(\lambda)$. 
Take $u \in \operatorname{Ann}_{\tilde{\mathcal{U}}} \tilde{V}(\lambda)$. Write $u=\sum_{i=1}^{m} u_{i} z_{i}$ where $u_{i} \in H, z_{i} \in \operatorname{Ann}_{A} \widetilde{V}(\lambda)$. Choose $z \in S$ such that $z u_{i} \in \widetilde{\mathcal{U}}$ and $z z_{i} \in \mathcal{Z}(\mathfrak{g})$ for all $i=1, \ldots, m$. Observe that $z z_{i} \in \operatorname{Ann}_{\mathcal{Z}(\mathfrak{g})} \widetilde{V}(\lambda)$ for all $i=1, \ldots, m$. Therefore

$$
z^{2} u=\sum_{i=1}^{m}\left(z u_{i}\right)\left(z z_{i}\right) \in \tilde{\mathcal{U}} \operatorname{Ann}_{\mathcal{Z}(\mathfrak{g})} \tilde{V}(\lambda)
$$

Recall that $S \cap \operatorname{Ann}_{\mathcal{Z}(\mathfrak{g})} \widetilde{V}(\lambda)=\emptyset$ and so $\left(z^{2}-c\right) \in \operatorname{Ann}_{\mathcal{Z}(\mathfrak{g})} \widetilde{V}(\lambda)$ for a certain non-zero scalar $c$. Hence $u \in \widetilde{\mathcal{U}} \operatorname{Ann}_{\mathcal{Z}(\mathfrak{g})} \widetilde{V}(\lambda)$. This completes the proof.

5.3. PRV determinants and Shapovalov form factorization. Assume that for some $\widetilde{V} \in \operatorname{Irr}$ the set $\operatorname{det} \mathcal{J}(\widetilde{V})$ contains a non-zero polynomial $p$ whose irreducible factors are all either a divisor of a Shapovalov form (see 2.5.8) or a divisor of $t$. An irreducible factor of a Shapovalov form takes either a form $\left(\alpha^{\vee}-(\rho, \alpha)-n\right)$ (a first type factor) for some non-isotropic positive root $\alpha$ and some $n \in \mathbb{N}^{+}$or a form $\left(\alpha^{\vee}-(\rho, \alpha)\right)$ (a second type factor) for some isotropic positive root $\alpha$. Note that the factors of the second type are divisors of $t$. Observe that for $n \neq 0$ the element

$$
s_{\alpha} \cdot\left(\alpha^{\vee}-(\rho, \alpha)-n\right)=-\alpha^{\vee}+(\rho, \alpha)-n
$$

is neither a divisor of a Shapovalov form nor a divisor of $t$. This forces the maximal $W$.-invariant factor of $p^{|W|}$ to be a divisor of $t^{m}$ for some $m \in \mathbb{N}$.

Then 4.7.4 4.7.5 imply

5.3.1. Theorem. Assume that for any $\widetilde{V} \in \operatorname{Irr}$ there exists a non-zero element in $\operatorname{det} \mathcal{J}(\widetilde{V})$ all of whose irreducible factors are either divisors of Shapovalov forms or divisors of $t$. Then there exists an ad $\mathfrak{g}$-submodule $H$ of $\widetilde{\mathcal{U}}$ such that

$$
H \cong \bigoplus_{\widetilde{V} \in \operatorname{Irr}} \mathrm{E}(\widetilde{V})^{\left.\oplus \operatorname{dim} \tilde{V}\right|_{0}}
$$

and the multiplication map induces an isomorphism $H \otimes \mathcal{Z}(\mathfrak{g})\left[T^{-2}\right] \stackrel{\sim}{\longrightarrow} \widetilde{\mathcal{U}}\left[T^{-2}\right]$.

Recall that $\widetilde{M}(\lambda)$ is simple iff all Shapovalov forms are non-zero at the point $\lambda$. Theorem 5.2 yields

5.3.2. Theorem. Assume that for any $\widetilde{V} \in \operatorname{Irr}$ there exists a non-zero element in $\operatorname{det} \mathcal{J}(\widetilde{V})$ all of whose irreducible factors are either divisors of Shapovalov forms or divisors of $t$. Then the annihilator of $\widetilde{M}(\lambda)$ is centrally generated provided $\widetilde{M}(\lambda)$ is simple strongly typical.

As shown in [GL1] the condition of the last two theorems holds for $\mathfrak{g}=\mathfrak{o s p}(1,2 n)$. We will prove that this condition also holds for the basic classical Lie superalgebras of type I. Contrary to the case $\mathfrak{o s p}(1,2 n)$, the proof is not based on the calculations of PRV determinants.

\section{The Basic Classical Lie Superalgebras of type I}

Throughout this section $\mathfrak{g}$ is a basic classical Lie superalgebra of type $\mathrm{I}$, that is, $\mathfrak{g}=\mathfrak{g l}(m, n), \mathfrak{s l}(m, n), \mathfrak{p s l}(n, n)$ or $\mathfrak{g}=\mathfrak{o s p}(2, n)$. We describe some common properties of these superalgebras which are used in the next section. 
6.1. Notation. The Lie superalgebras of type I are $\mathbb{Z}$-graded. We denote by $\mathfrak{g}_{r}$ $(r \in \mathbb{Z})$ the corresponding homogeneous component of $\mathfrak{g}$, and by $\mathfrak{g}_{\overline{0}}$ (resp., $\mathfrak{g}_{\overline{1}}$ ) the even (resp., the odd) part of $\mathfrak{g}$.

One has $\mathfrak{g}_{0}=\mathfrak{g}_{0}$ and $\mathfrak{g}_{\overline{1}}=\mathfrak{g}_{1}+\mathfrak{g}_{-1}$. In particular, both superalgebras $\mathfrak{g}_{ \pm 1}$ are supercommutative and the exterior algebras $\Lambda \mathfrak{g}_{ \pm 1}$ are naturally embedded into $\tilde{\mathcal{U}}$. One has $\tilde{\mathcal{U}}=\Lambda\left(\mathfrak{g}_{-1}\right) \mathcal{U} \Lambda\left(\mathfrak{g}_{1}\right)$. As ad $\mathfrak{g}_{0}$-modules $\mathfrak{g}_{ \pm 1}$ are irreducible and dual one to another.

All odd roots are isotropic and so the notion of "typical" coincides with the notion of "strongly typical".

Extend the above $\mathbb{Z}$-grading on $U(\mathfrak{g})$; evidently $U(\mathfrak{g})_{r}=0$ if $|r|>\operatorname{dim} \mathfrak{g}_{1}$. Moreover for $r=\operatorname{dim} \mathfrak{g}_{1}$ one has $U(\mathfrak{g})_{ \pm r}=\mathcal{U} \Lambda^{r}\left(\mathfrak{g}_{ \pm 1}\right)$. By default, "the degree" of an element of $\widetilde{\mathcal{U}}$ means its degree with respect to this $\mathbb{Z}$-grading.

6.1.1. Distinguished triangular decomposition. Set $\mathfrak{n}_{d}^{ \pm}:=\mathfrak{n}_{0}^{ \pm}+\mathfrak{g}_{ \pm 1}$. The decomposition $\mathfrak{g}=\mathfrak{n}_{d}^{-} \oplus \mathfrak{h} \oplus \mathfrak{n}_{d}^{+}$is called a distinguished triangular decomposition. By default, all highest weight modules, positive/negative roots, Harish-Chandra projection $\mathcal{P}$ and PRV matrices/determinants are constructed with respect to the distinguished triangular decomposition.

Note that $(\rho, \alpha)=\left(\rho_{0}, \alpha\right)$ for any $\alpha \in \Delta_{0}$ since $\Delta_{1}^{+}=\Omega\left(\mathfrak{g}_{1}\right)$ is $W$-invariant. In particular, $w \cdot \mu=w\left(\mu+\rho_{0}\right)-\rho_{0}$ for any $w \in W$ and $\mu \in \mathfrak{h}^{*}$.

The Verma modules constructed using a distinguished triangular decomposition have the following nice structure: $\widetilde{M}(\mu)=\operatorname{Ind}_{\mathfrak{g}_{0} \oplus \mathfrak{n}_{1}^{+}}^{\mathfrak{g}} M(\mu)$ where the action of $\mathfrak{n}_{1}^{+}$ on $M(\mu)$ is assumed to be trivial.

6.1.2. For $\alpha \in \Delta_{0}^{+}$denote by $e_{\alpha}$ (resp., $f_{\alpha}$ ) an element of the weight $\alpha$ (resp., $-\alpha$ ) of $\mathfrak{g}$. For $\beta \in \Delta_{1}^{+}$(resp., $\beta \in \Delta_{1}^{-}$) denote by $x_{\beta}$ (resp., $y_{\beta}$ ) an element of the weight $\beta$ of $\mathfrak{g}$.

Denote by $I$ the set of the positive odd roots with a fixed total order. Then $\left\{x_{i}\right\}_{i \in I}$ (resp., $\left\{y_{i}\right\}_{i \in I}$ ) is a basis of $\mathfrak{g}_{1}$ (resp., $\mathfrak{g}_{-1}$ ). For any $J \subseteq I$ set $x_{J}:=\prod_{i \in J} x_{i}$, $y_{J}:=\prod_{i \in J} y_{i}$ where the products are taken with respect to the total order on $I$. If we change the order of factors in the product $y_{J}$, then the result is equal either to $y_{J}$ or to $\left(-y_{J}\right)$, since $\mathfrak{g}_{ \pm 1}$ are supercommutative. Note that $y y_{I}=x x_{I}=0$ for any $y \in \mathfrak{g}_{-1}, x \in \mathfrak{g}_{1}$.

Evidently $x_{I} \in \Lambda^{t o p} \mathfrak{g}_{1}, y_{I} \in \Lambda^{t o p} \mathfrak{g}_{-1}$ and so $x_{I}, y_{I}$ are invariant with respect to the adjoint action of $\left[\mathfrak{g}_{0}, \mathfrak{g}_{0}\right]$. Moreover $x_{I} y_{I}$ is ad $\mathfrak{g}_{0}$-invariant due to the duality $\mathfrak{g}_{1}^{*} \cong \mathfrak{g}_{-1}$.

6.1.3. Sometimes we will deal with a non-distinguished triangular decomposition $\mathfrak{g}=\mathfrak{n}^{-} \oplus \mathfrak{h} \oplus \mathfrak{n}^{+}$. We shall use the following notation: $\mathfrak{b}:=\mathfrak{h}+\mathfrak{n}^{+} ; \mathcal{P}_{\mathfrak{b}}$ will be the Harish-Chandra projection with respect to this triangular decomposition, $\Delta(\mathfrak{b})$ will be the set of non-zero roots of $\mathfrak{b}$ and also

$$
\begin{aligned}
I_{+} & :=I \cap \Delta(\mathfrak{b}), \\
I_{-} & :=I \backslash I_{+}, \\
t_{\mathfrak{b}} & :=\mathcal{P}_{\mathfrak{b}}(T) .
\end{aligned}
$$

Note that

$$
t_{\mathfrak{b}}:=\prod_{\beta \in \Delta_{1} \cap \Delta_{\mathfrak{b}}}\left(\beta^{\vee}+(\beta, \rho)\right)
$$


As before, the triangular decomposition of $\mathfrak{g}_{0}$ is assumed to be fixed and all triangular decompositions of $\mathfrak{g}$ which we consider are compatible with this triangular decomposition of $\mathfrak{g}_{0}$.

6.1.4. Case $\mathfrak{p s l}(n, n)$. This case is rather special. A Cartan algebra of $\mathfrak{g}=\mathfrak{p s t}(n, n)$ is "too small" and a distinguished triangular decomposition does not fit the definition given in 2.2. Moreover the restriction of the Harish-Chandra projection determined by the distinguished triangular decomposition to the zero weight space is not an algebra homomorphism: for instance, both $y_{I}, x_{I}$ have zero weight, $\mathcal{P}\left(y_{I}\right)=\mathcal{P}\left(x_{I}\right)=0$ however $\mathcal{P}\left(x_{I} y_{I}\right)=t$ - see Corollary 6.3.3.

A possible treatment to this problem is the following "enlargement of a Cartan subsuperalgebra". The Lie superalgebra $\mathfrak{g}$ is an ideal in the Lie superalgebra $\hat{\mathfrak{g}}:=$ $\mathfrak{p g l}(n, n)=\mathfrak{g l}(n, n) /(\mathbb{C} X)$ where $X$ stands for the identity matrix. One has $\hat{\mathfrak{g}}_{\overline{1}}=\mathfrak{g}_{\overline{1}}$ and $\hat{\mathfrak{g}}_{0}=\mathfrak{g}_{0} \times \mathbb{C} z$ where $z$ is a central element of the Lie algebra $\hat{\mathfrak{g}}_{0}$.

Let $\hat{\mathfrak{h}}$ be a Cartan subsuperalgebra of $\hat{\mathfrak{g}}_{0}$ spanned by $\mathfrak{h}$ and $z$. One can easily see that $\hat{\mathfrak{h}}$ acts semisimply on $\mathfrak{g}$ and a distinguished triangular decomposition of $\mathfrak{g}$ is determined, in the sense of 2.2, by a certain regular element $h \in \hat{\mathfrak{h}}$. For $\mu \in \hat{\mathfrak{h}}^{*}$ set $\left.\widetilde{\mathcal{U}}\right|_{\mu}:=\{u \in \widetilde{\mathcal{U}} \mid[h, u]=\mu(h) u, \forall h \in \hat{\mathfrak{h}}\}$. Then the restriction of the HarishChandra projection determined by the distinguished triangular decomposition on $\left.\widetilde{\mathcal{U}}\right|_{0}$ is an algebra homomorphism because $\left.\widetilde{\mathcal{U}}\right|_{0} \cap \mathcal{U}\left(\mathfrak{n}^{-}+\mathfrak{h}\right) \mathfrak{n}^{-}=0$.

It is easy to see that for any weight $\mathfrak{g}$-module $M$ one can extend (not uniquely) its $\mathfrak{g}$-module structure to a $\hat{\mathfrak{g}}$-module structure. This implies that Lemma 4.1.1 remains true for the distinguished triangular decomposition if we define $\left.\widetilde{\mathcal{U}}\right|_{0}$ as above. For ad $\mathfrak{g}$-submodules of $\widetilde{\mathcal{U}}$ consider the natural $\hat{\mathfrak{g}}$-module structure coming from the embedding $\widetilde{\mathcal{U}}$ into $\mathcal{U}(\hat{\mathfrak{g}})$. In the sequel, we substitute the categories Irr and $\widetilde{\mathcal{O}}$ for $\mathfrak{g}$ by the same categories for $\hat{\mathfrak{g}}$ (these $\hat{\mathfrak{g}}$-categories have the same sets of objects as their $\mathfrak{g}$-analogues but less morphisms). Under these conventions all propositions of Sections 4 and 5 remain true for $\mathfrak{g}=\mathfrak{p s r}(n, n)$.

6.2. Useful assertions. The following lemma is an immediate consequence of the supercommutativity of $\mathfrak{g}_{ \pm 1}$.

6.2.1. Lemma. Let $\widetilde{N}$ be a $\mathfrak{g}$-module, and let $N$ be its $\mathfrak{g}_{0}$-submodule such that $\widetilde{N}=\widetilde{\mathcal{U}} N$. Then the canonical map $\operatorname{Ind}_{\mathfrak{g}_{0}}^{\mathfrak{g}} N \rightarrow \widetilde{N}$ is an isomorphism iff for each collection $\left\{v_{J}\right\}_{J \subseteq I}$ of elements of $N$ one has

$$
x_{I} \sum_{J \subseteq I} y_{J} v_{J}=0 \Longrightarrow v_{J}=0, \forall J \subseteq I .
$$

Recall that $\widetilde{M}(\lambda)$ is typical iff $t(\lambda) \neq 0$ or, equivalently, $T \widetilde{M}(\lambda) \neq 0$.

6.2.2. Lemma. A typical Verma module $\widetilde{M}(\lambda)$ contains a simple Verma submodule.

Proof. Recall that $\left(\rho_{0}, \alpha\right)=(\rho, \alpha)$ for any $\alpha \in \Delta_{0}$. Comparing the factorizations of Shapovalov forms (see 2.5.8) for the Lie superalgebras $\mathfrak{g}$ and $\mathfrak{g}_{0}$, one concludes that a typical $\mathfrak{g}$-module $\widetilde{M}(\lambda)$ is simple iff the $\mathfrak{g}_{0}$-module $M(\lambda)$ is simple. Let $\widetilde{M}(\lambda)$ be a typical Verma module, and let $v$ be its highest weight vector. Then $\mathcal{U}\left(\mathfrak{n}_{0}^{-}\right) v \cong M(\lambda)$ contains a simple submodule $M\left(\lambda^{\prime}\right)$. Therefore there exists $u \in \mathcal{U}\left(\mathfrak{n}_{0}^{-}\right)$such that $\mathfrak{n}_{0}^{+} u v=0$. The ad $\mathfrak{g}_{0}$-invariance of $\mathfrak{g}_{1}$ yields $\mathfrak{g}_{1}(u v)=u \mathfrak{g}_{1} v=0$. Thus the vector $u v$ is primitive and so $\widetilde{\mathcal{U}}(u v)$ is a quotient of $\widetilde{M}\left(\lambda^{\prime}\right)$. The non-zero elements of $\mathcal{U}\left(\mathfrak{g}_{0}\right)$ 
are non-zero divisors in $\widetilde{\mathcal{U}}$ since the non-zero elements of $\mathcal{S}\left(\mathfrak{g}_{0}\right)$ are non-zero divisors in $\mathcal{S}(\mathfrak{g})=\mathcal{S}\left(\mathfrak{g}_{0}\right) \Lambda\left(\mathfrak{g}_{\overline{1}}\right)$. Thus $\widetilde{\mathcal{U}}(u v) \cong \widetilde{M}\left(\lambda^{\prime}\right)$. Since $M\left(\lambda^{\prime}\right)$ is simple, $\widetilde{M}\left(\lambda^{\prime}\right)$ is also simple. The lemma is proved.

6.3. Element $T$. For the classical Lie superalgebras of type I the element $T$ takes a very simple form given by the following

6.3.1. Lemma. Up to a non-zero scalar $T=\left(\operatorname{ad}^{\prime} y_{I} x_{I}\right) 1$.

Proof. Recall 3.3.2. It is enough to show that if $v$ is a generator of a trivial $\mathfrak{g}_{0^{-}}$ module $V(0)$, then $y_{I} x_{I} v$ spans a trivial $\mathfrak{g}$-submodule of $\operatorname{Ind}_{\mathfrak{g}_{0}}^{\mathfrak{g}} V(0)$. In other words, one has to verify that $\mathfrak{g}\left(y_{I} x_{I}\right) \subset \widetilde{\mathcal{U}} \mathfrak{g}_{0}$. The ad $\mathfrak{g}_{0}$-invariance of $y_{I} x_{I}$ forces $\mathfrak{g}_{0}\left(y_{I} x_{I}\right)=$ $\left(y_{I} x_{I}\right) \mathfrak{g}_{0}$. Moreover $\mathfrak{g}_{-1}\left(y_{I} x_{I}\right)=0$. Thus it remains to check that $x_{\beta} y_{I} x_{I} \in \widetilde{\mathcal{U}} \mathfrak{g}_{0}$ for any $\beta \in \Delta_{1}^{+}$. Setting $J:=I \backslash\{\beta\}$ one has

$$
\begin{aligned}
x_{\beta} y_{I} x_{I} & = \pm x_{\beta} y_{-\beta} y_{J} x_{I}= \pm\left(\left[x_{\beta}, y_{-\beta}\right]-y_{-\beta} x_{\beta}\right) y_{J} x_{I} \\
& = \pm\left(\left[x_{\beta}, y_{-\beta}\right] y_{J} x_{I}-y_{-\beta}\left(\left(\operatorname{ad} x_{\beta}\right), y_{J}\right) x_{I}\right)
\end{aligned}
$$

since $x_{\beta} x_{I}=0$.

The term $y_{I} x_{I}$ is of zero weight and so the weight of $y_{J} x_{I}$ is equal to $\beta$. The term $\left[x_{\beta}, y_{-\beta}\right]$ lies in $\mathfrak{h}$; one has $\left[\left[x_{\beta}, y_{-\beta}\right], x_{\beta}\right]=0$ since $x_{\beta}^{2}=0$. Therefore $\beta\left(\left[x_{\beta}, y_{-\beta}\right]\right)=$ 0 and so $\left[x_{\beta}, y_{-\beta}\right] y_{J} x_{I}=y_{J} x_{I}\left[x_{\beta}, y_{-\beta}\right] \in \widetilde{\mathcal{U}} \mathfrak{g}_{0}$.

Let us show that $y_{-\beta}\left(\left(\operatorname{ad} x_{\beta}\right) y_{J}\right) x_{I} \in \widetilde{\mathcal{U}} \mathfrak{g}_{0}$. For any $\beta^{\prime} \in J$ one has $\left(\operatorname{ad} x_{\beta}\right) y_{-\beta^{\prime}} \in$ $\left(\mathfrak{n}_{0}^{-}+\mathfrak{n}_{0}^{+}\right)$. Since $\Lambda^{r}\left(\mathfrak{g}_{-1}\right)$ is ad $\mathfrak{g}_{0}$-invariant for all $r$, this implies that

$$
\left(\operatorname{ad} x_{\beta}\right) y_{J} \in \Lambda^{l-2}\left(\mathfrak{g}_{-1}\right)\left(\mathbb{C}+\mathfrak{n}_{0}^{-}+\mathfrak{n}_{0}^{+}\right)
$$

where $l:=\operatorname{dim} \mathfrak{g}_{1}$. If $y_{J^{\prime}} \in \Lambda^{l-2}\left(\mathfrak{g}_{-1}\right)$ has the same weight as the element $\left(\operatorname{ad} x_{\beta}\right) y_{J}$, then the set $I \backslash J^{\prime}$ contains two elements whose sum is equal to $2 \beta$. Since the multiset $I$ contains exactly one element equal to $\beta$, this implies $\beta \in J^{\prime}$, that is, $y_{-\beta} y_{J^{\prime}}=0$. Then (6) gives

$$
y_{-\beta}\left(\left(\operatorname{ad} x_{\beta}\right) y_{J}\right) \in y_{-\beta} \Lambda^{l-2}\left(\mathfrak{g}_{-1}\right)\left(\mathfrak{n}_{0}^{-}+\mathfrak{n}_{0}^{+}\right)
$$

and so

$$
y_{-\beta}\left(\left(\operatorname{ad} x_{\beta}\right) y_{J}\right) x_{I} \in \Lambda^{l-1}\left(\mathfrak{g}_{-1}\right)\left(\mathfrak{n}_{0}^{-}+\mathfrak{n}_{0}^{+}\right) x_{I}=\Lambda^{l-1}\left(\mathfrak{g}_{-1}\right) x_{I}\left(\mathfrak{n}_{0}^{-}+\mathfrak{n}_{0}^{+}\right) \in \widetilde{\mathcal{U}} \mathfrak{g}_{0}
$$

since $x_{I}$ is $\operatorname{ad}\left(\left[\mathfrak{g}_{0}, \mathfrak{g}_{0}\right]\right.$-invariant. The assertion follows.

Take any triangular decomposition $\mathfrak{g}=\mathfrak{n}^{-} \oplus \mathfrak{h} \oplus \mathfrak{n}^{+}$and retain the notation of 6.1 .3

6.3.2. Lemma. Up to a non-zero scalar one has

$$
\mathcal{P}_{\mathfrak{b}}\left(x_{I_{+}} y_{I_{+}}\right) \mathcal{P}_{\mathfrak{b}}\left(y_{I_{-}} x_{I_{-}}\right)=\prod_{\beta \in \Delta_{1}(\mathfrak{b})}\left(\beta^{\vee}+\beta^{\vee}\left(\rho_{\mathfrak{b}}\right)\right)
$$

Proof. The right-hand side is equal to $t_{\mathfrak{b}}=\mathcal{P}_{\mathfrak{b}}(T)$. By Lemma 6.3.1, up to a non-zero scalar $T=\left(\operatorname{ad}^{\prime} x_{I} y_{I}\right) 1$. Thus one should verify that

$$
\mathcal{P}_{\mathfrak{b}}\left(\left(\operatorname{ad}^{\prime} y_{I} x_{I}\right) 1\right)=\mathcal{P}_{\mathfrak{b}}\left(x_{I_{+}} y_{I_{+}}\right) \mathcal{P}_{\mathfrak{b}}\left(y_{I_{-}} x_{I_{-}}\right)
$$

up to a non-zero scalar.

Since $\mathfrak{g}_{1}$ is supercommutative, $\left(\operatorname{ad}^{\prime} x_{I}\right) 1=2^{\operatorname{dim} \mathfrak{g}_{1}} x_{I}$ and

$$
\mathcal{P}_{\mathfrak{b}}\left(\left(\operatorname{ad}^{\prime} y_{I} x_{I}\right) 1\right)=2^{\operatorname{dim} \mathfrak{g}_{1}} \mathcal{P}_{\mathfrak{b}}\left(\left(\operatorname{ad}^{\prime} y_{I}\right) x_{I}\right)= \pm 2^{\operatorname{dim} \mathfrak{g}_{1}} \mathcal{P}_{\mathfrak{b}}\left(\left(\operatorname{ad}^{\prime} y_{I_{+}} \operatorname{ad}^{\prime} y_{I_{-}}\right) x_{I}\right)
$$


Recall that for $\alpha \in I_{+}$one has $y_{-\alpha} \in \mathfrak{n}^{-}$and so $\mathcal{P}\left(\left(a d^{\prime} y_{-\alpha}\right) u\right)= \pm \mathcal{P}\left(u y_{-\alpha}\right)$ for all $u \in \tilde{\mathcal{U}}$. Therefore, up to a non-zero scalar,

$$
\mathcal{P}_{\mathfrak{b}}\left(\left(\operatorname{ad}^{\prime} y_{I} x_{I}\right) 1\right)=\mathcal{P}_{\mathfrak{b}}\left(\left(\left(\operatorname{ad}^{\prime} y_{I_{-}}\right) x_{I}\right) y_{I_{+}}\right) .
$$

Similarly for any $\alpha \in I_{-}$one has $y_{-\alpha} \in \mathfrak{n}^{+}$and so $\mathcal{P}_{\mathfrak{b}}\left(u y_{-\alpha} y_{I_{+}}\right)=\mathcal{P}_{\mathfrak{b}}\left( \pm u y_{I_{+}} y_{-\alpha}\right)=$ 0 . Therefore

$$
\begin{aligned}
\mathcal{P}_{\mathfrak{b}}\left(\left(\left(\operatorname{ad}^{\prime} y_{I_{-}}\right) x_{I}\right) y_{I_{+}}\right) & =\mathcal{P}_{\mathfrak{b}}\left(y_{I_{-}} x_{I} y_{I_{+}}\right)= \pm \mathcal{P}_{\mathfrak{b}}\left(y_{I_{-}} x_{I_{-}} x_{I_{+}} y_{I_{+}}\right) \\
& = \pm \mathcal{P}_{\mathfrak{b}}\left(y_{I_{-}} x_{I_{-}}\right) \mathcal{P}_{\mathfrak{b}}\left(x_{I_{+}} y_{I_{+}}\right)
\end{aligned}
$$

where the last equality is a consequence of the fact that the restriction of the Harish-Chandra projection to the zero weight space is an algebra homomorphism. Hence

$$
\mathcal{P}_{\mathfrak{b}}\left(\left(\operatorname{ad}^{\prime} y_{I} x_{I}\right) 1\right)= \pm 2^{\operatorname{dim} \mathfrak{g}_{1}} \mathcal{P}_{\mathfrak{b}}\left(x_{I_{+}} y_{I_{+}}\right) \mathcal{P}_{\mathfrak{b}}\left(y_{I_{-}} x_{I_{-}}\right) .
$$

The lemma is proved.

6.3.3. Corollary. Up to a non-zero scalar one has

$$
\mathcal{P}\left(x_{I} y_{I}\right)=t=\prod_{\beta \in \Delta_{1}^{+}}\left(\beta^{\vee}+\beta^{\vee}(\rho)\right) .
$$

6.3.4. Lemma. If $\lambda \in \mathfrak{h}^{*}$ is such that $\mathcal{P}_{\mathfrak{b}}\left(x_{I_{+}} y_{I_{+}}\right)(\lambda)=0$, then $x_{I} \widetilde{M}_{\mathfrak{b}}(\lambda)=0$. If $\mathcal{P}_{\mathfrak{b}}\left(y_{I_{-}} x_{I_{-}}\right)(\lambda)=0$, then $y_{I} \widetilde{M}_{\mathfrak{b}}(\lambda)=0$.

Proof. Both assertions are similar. To verify the first one, fix $\lambda$ such that

$$
\mathcal{P}_{\mathfrak{b}}\left(x_{I_{+}} y_{I_{+}}\right)(\lambda)=0
$$

and set $\widetilde{M}:=\widetilde{M}_{\mathfrak{b}}(\lambda)$. Denote by $v$ a highest weight vector of $\widetilde{M}$. One has

$$
x_{I} \widetilde{M}=x_{I} \mathcal{U}\left(\mathfrak{n}_{0}^{-}\right) \sum_{J_{1} \subseteq I_{+}, J_{2} \subseteq I_{-}} x_{J_{2}} y_{J_{1}} v=\mathcal{U}\left(\mathfrak{n}_{0}^{-}\right) \sum_{J_{1} \subseteq I_{+}} x_{I} y_{J_{1}} v
$$

since $x_{I}$ is ad $\mathfrak{n}_{0}^{-}$-invariant and $x_{I} x_{J}=0$ for $J \neq \emptyset$. Note that $\widetilde{\mathcal{U}}_{k} v=0$ if $k>\# I_{-}$. Therefore $x_{I} y_{J_{1}} v=0$ if $\left(\# I-\# J_{1}\right)>\# I_{-}$, that is, if $\# J_{1}<\# I_{+}$. Consequently

$$
x_{I} \widetilde{M}=\mathcal{U}\left(\mathfrak{n}_{0}^{-}\right) x_{I} y_{I_{+}} v=\mathcal{U}\left(\mathfrak{n}_{0}^{-}\right) x_{I_{-}} x_{I_{+}} y_{I_{+}} v=\mathcal{U}\left(\mathfrak{n}_{0}^{-}\right) x_{I_{-}} \mathcal{P}_{\mathfrak{b}}\left(x_{I_{+}} y_{I_{+}}\right)(\lambda) v=0
$$

as required.

\section{Separation and Annihilation Theorems for the type I case}

Throughout this section $\mathfrak{g}$ is a basic classical Lie superalgebra of type I, that is, $\mathfrak{g}=\mathfrak{g l}(m, n), \mathfrak{s l}(m, n), \mathfrak{p s l}(n, n)$ or $\mathfrak{g}=\mathfrak{o s p}(2, n)$. In this section we establish separation and annihilation type theorems for these algebras.

Retain the notation of Section 6 Until 7.3.2 we will deal only with a distinguished triangular decomposition.

7.1. Proposition. For a typical Verma module $\widetilde{M}$ one has

(i) $\quad F(\widetilde{M}, \widetilde{M}) \cong \bigoplus_{V \in \operatorname{Irr}_{0}}\left(\operatorname{Ind}_{\mathfrak{g} 0}^{\mathfrak{g}} V\right)^{\left.\oplus \operatorname{dim} V\right|_{0}}$.

(ii) For $H$ a harmonic space of $\mathcal{U}\left(\mathfrak{g}_{0}\right)$ the restriction of the natural map $\widetilde{\mathcal{U}} \rightarrow F(\widetilde{M}, \widetilde{M})$ to $(\operatorname{ad} \widetilde{\mathcal{U}})\left(H x_{I} y_{I}\right)$ is a bijection. 
Proof. Let $H$ be a harmonic space of $\mathcal{U}\left(\mathfrak{g}_{0}\right)$. Recall that $\operatorname{dim}_{\mathfrak{g}_{0}}(V, H)=\left.\operatorname{dim} V\right|_{0}$ for any $V \in \operatorname{Irr}_{0}$.

Define the map $\psi: \operatorname{Ind}_{\mathfrak{g}_{0}}^{\mathfrak{g}}(H) \rightarrow(\operatorname{ad} \tilde{\mathcal{U}})\left(H x_{I} y_{I}\right)$ by $g \otimes h \mapsto(\operatorname{ad} g)\left(h x_{I} y_{I}\right)$. Since $x_{I} y_{I}$ is ad $\mathfrak{g}_{0}$-invariant, $H x_{I} y_{I} \cong H$ as ad $\mathfrak{g}_{0}$-modules and so $\psi$ is a $\mathfrak{g}$-epimorphism. Denote by $f$ the natural map $f: \widetilde{\mathcal{U}} \rightarrow F(\widetilde{M}, \widetilde{M})$. To prove the theorem it is enough to show that the map $f \circ \psi: \operatorname{Ind}_{\mathfrak{g}_{0}}^{\mathfrak{g}}\left(H x_{I} y_{I}\right) \rightarrow F(\widetilde{M}, \widetilde{M})$ is a bijection.

Let $\mu$ be the highest weight of $\widetilde{M}$, and let $v$ be a highest weight vector of $\widetilde{M}$. Then $M:=\mathcal{U}\left(\mathfrak{g}_{0}\right) v \cong M(\mu)$. By 2.8.2, the restriction of the natural map $\mathcal{U}\left(\mathfrak{g}_{0}\right) \rightarrow F(M, M)$ to $H$ is bijective.

To prove that $f \circ \psi$ is a monomorphism recall Lemma 6.2.1. Let $\left\{a_{J}\right\}_{J \subseteq I} \subset H$ be such that

$$
b \widetilde{M}=0 \quad \text { where } \quad b:=\left(\sum_{J \subseteq I}\left(\operatorname{ad} x_{I} y_{J}\right)\left(a_{J} x_{I} y_{I}\right)\right) .
$$

We need to show that $a_{J}=0$ for all $J \subseteq I$. One has $\left(\operatorname{ad} y_{J}\right)\left(a_{J} x_{I} y_{I}\right)=y_{J} a_{J} x_{I} y_{I}$ since $\mathfrak{g}_{-1} y_{I}=0$. Therefore

$$
b=\left(\operatorname{ad} x_{I}\right)\left(\sum_{J \subseteq I} y_{J} a_{J} x_{I} y_{I}\right) .
$$

Take $u \in \mathcal{U}\left(\mathfrak{n}_{0}^{-}\right)$. The equality $\mathfrak{g}_{-1} y_{I}=0$ implies

$$
0=b\left(y_{I} u v\right)=\left(\left(\operatorname{ad} x_{I}\right)\left(\sum_{J \subseteq I} y_{J} a_{J} x_{I} y_{I}\right)\right) y_{I} u v=\sum_{J \subseteq I} \pm y_{J} a_{J} x_{I} y_{I} x_{I} y_{I} u v
$$

since $y_{I} x_{J^{\prime}} y_{I}=0$ for $J^{\prime} \neq I$ (the degree of $y_{I} x_{J^{\prime}} y_{I}$ is equal to $r:=\# J^{\prime}-\# 2 I$ and $\widetilde{\mathcal{U}}_{r}=0$ for $\left.r<-\# I\right)$. By Corollary 6.3.3. $\mathcal{P}\left(x_{I} y_{I}\right)=t$ and so $x_{I} y_{I} u v=$ $u x_{I} y_{I} v=t(\mu) u v$. Therefore $b\left(y_{I} u v\right)=t(\mu)^{2} \sum_{J \subseteq I} \pm y_{J} a_{J} u v$. Hence $b\left(y_{I} M\right)=$ $t(\mu)^{2} \sum_{J \subseteq I} \pm y_{J} a_{J} M$. Since $t(\mu) \neq 0$ and $a_{J} M \subset M$, the equality $b\left(y_{I} M\right)=0$ implies $a_{J} M=0$ for all $J \subseteq I$. However the $a_{J}$ belong to $H$ and $H \cap$ Ann $M=0$ by Theorem 2.8.2 Therefore all $a_{J}$ are equal to zero. Hence $f \circ \psi$ is a monomorphism.

Let us show that $f \circ \psi$ is surjective. Recall that as a $\mathfrak{g}_{0}$-module $\widetilde{M} \cong M \otimes$ $\Lambda \mathfrak{g}_{-1}$. Take a simple finite-dimensional $\mathfrak{g}_{0}$-module $V$. Using Frobenius reciprocity and 2.8.2 one obtains

$$
\begin{aligned}
& \operatorname{dim} \operatorname{Hom}_{\mathfrak{g}_{0}}(V, F(\widetilde{M}, \widetilde{M}))=\operatorname{dim} \operatorname{Hom}_{\mathfrak{g}_{0}}(\widetilde{M} \otimes V, \widetilde{M}) \\
& =\operatorname{dim} \operatorname{Hom}_{\mathfrak{g}_{0}}\left(V \otimes \Lambda \mathfrak{g}_{-1} \otimes M, \Lambda \mathfrak{g}_{-1} \otimes M\right) \\
& =\operatorname{dim} \operatorname{Hom}_{\mathfrak{g}_{0}}\left(V \otimes \Lambda \mathfrak{g}_{\overline{1}}, F(M, M)\right) \\
& =\operatorname{dim} \operatorname{Hom}_{\mathfrak{g}_{0}}\left(V \otimes \Lambda \mathfrak{g}_{\overline{1}}, H\right)=\operatorname{dim} \operatorname{Hom}_{\mathfrak{g}_{0}}\left(V, \Lambda \mathfrak{g}_{\mathrm{T}} \otimes H\right) \\
& =\operatorname{dim} \operatorname{Hom}_{\mathfrak{g}_{0}}\left(V, \operatorname{Ind}_{\mathfrak{g}_{0}}^{\mathfrak{g}} H\right)=[\operatorname{Im}(f \circ \psi): V] \text {. }
\end{aligned}
$$

Since $F(\widetilde{M}, \widetilde{M})$ is a completely reducible ad $\mathfrak{g}_{0}$-module and the multiplicity $[\operatorname{Im}(f \circ \psi): V]$ is finite, this gives $F(\widetilde{M}, \widetilde{M})=\operatorname{Im}(f \circ \psi)$ and completes the proof. 
Retain the notation of 4.6 .2

7.1.1. Corollary. Let $H$ be a harmonic space of $\mathcal{U}\left(\mathfrak{g}_{0}\right)$ and $L:=(\operatorname{ad} \widetilde{\mathcal{U}})\left(H x_{I} y_{I}\right)$. Then

$$
L \cong \bigoplus_{\tilde{V} \in \operatorname{Irr}} \mathrm{E}(\tilde{V})^{\left.\oplus \operatorname{dim} \tilde{V}\right|_{0}}
$$

and for any $\widetilde{V} \in \operatorname{Irr}$ the determinant $\operatorname{det} \mathcal{J}(\widetilde{V} ; L)$ admits a linear factorization. Moreover each linear factor is a factor of a Shapovalov form.

Proof. Combining Proposition 7.1 and (3) one obtains

$$
L \cong \bigoplus_{\widetilde{V} \in \operatorname{Irr}_{f}^{0}} \mathrm{E}(\widetilde{V})^{\left.\oplus \operatorname{dim} \tilde{V}\right|_{0}}
$$

Fix $\widetilde{V} \in \operatorname{Irr}$ and set $p:=P R V(\widetilde{V} ; L)$. Recall that $\widetilde{M}(\mu)$ is simple iff $\mu$ is not a zero of a Shapovalov form. In particular, an atypical Verma module is not simple. Taking into account Corollary 4.1.2, one concludes from Proposition 7.1 that $p(\mu) \neq 0$ provided that $\widetilde{M}(\mu)$ is simple. Thus any zero of $p$ is a zero of a Shapovalov form. Since each Shapovalov form admits a factorization into linear factors, this implies that the set of zeroes of $p$ lies in a union of countably many hyperplanes which correspond to the linear factors of the Shapovalov forms. Therefore $p$ is a product of linear factors which are factors of Shapovalov forms.

7.2. Separation theorem. Combining Corollary 7.1.1, Theorem 4.7.4, Corollary 4.7 .7 and 5.3 one obtains the following version of the Separation Theorem

7.2.1. Theorem. Let $H$ be a harmonic space of $\mathcal{U}\left(\mathfrak{g}_{0}\right)$. Then the multiplication map provides an isomorphism $\left((\operatorname{ad} \widetilde{\mathcal{U}}) H x_{I} y_{I}\right) \otimes \mathcal{Z}(\mathfrak{g})\left[T^{-2}\right] \stackrel{\sim}{\longrightarrow} \widetilde{\mathcal{U}}\left[T^{-2}\right]$.

7.3. Annihilation theorem. In this subsection we prove that $\operatorname{Ann} \widetilde{M}_{\mathfrak{b}}(\lambda)$ is a centrally generated ideal iff $\widetilde{M}_{\mathfrak{b}}(\lambda)$ is typical.

7.3.1. Combining Corollary 7.1.1 and Theorem 5.3.2, one concludes that Ann $\widetilde{M}(\lambda)$ is centrally generated if $\widetilde{M}(\lambda)$ is simple. By Lemma 6.2.2 any typical Verma module $\widetilde{M}(\lambda)$ contains a simple Verma submodule. This implies that $\operatorname{Ann} \widetilde{M}(\lambda)$ is centrally generated if $\widetilde{M}(\lambda)$ is typical. Using 2.7, it is easy to generalize this statement to any Borel subsuperalgebra $\mathfrak{b}$. Indeed, a typical Verma module $\widetilde{M}_{\mathfrak{b}}(\lambda)$ is isomorphic to a Verma module $\widetilde{M}\left(\lambda^{\prime}\right)$. Recall that a Verma module $\widetilde{M}_{\mathfrak{b}}(\lambda)$ is typical iff its annihilator does not contain $T$ - see 3.3.2 Thus $\widetilde{M}\left(\lambda^{\prime}\right)$ is typical and so Ann $\widetilde{M}_{\mathfrak{b}}(\lambda)=\operatorname{Ann} \widetilde{M}\left(\lambda^{\prime}\right)$ is a centrally generated ideal. Hence Ann $\widetilde{M}_{\mathfrak{b}}(\lambda)$ is centrally generated provided that $\widetilde{M}_{\mathfrak{b}}(\lambda)$ is typical.

In the rest of this section we will show that $\operatorname{Ann} \widetilde{M}_{\mathfrak{b}}(\lambda) \neq \widetilde{\mathcal{U}} \operatorname{Ann}_{\mathcal{Z}(\mathfrak{g})} \widetilde{M}_{\mathfrak{b}}(\lambda)$ if $\widetilde{M}_{\mathfrak{b}}(\lambda)$ is atypical, that is, $t_{\mathfrak{b}}(\lambda)=0$.

7.3.2. The proof goes as follows. Take $\lambda \in \mathfrak{h}^{*}$ such that $t_{\mathfrak{b}}(\lambda)=0$. Set $\tilde{\chi}:=$ $\operatorname{Ann}_{\mathcal{Z}(\mathfrak{g})} \widetilde{M}_{\mathfrak{b}}(\lambda)$. The idea is to find $\widetilde{V} \in \operatorname{Irr}$ satisfying the following conditions:
a) $\operatorname{Hom}_{\mathfrak{g}}(\widetilde{V}, \widetilde{\mathcal{U}}) \neq 0$,
b) $\widetilde{V}$ is typical,
c) $\forall \theta \in \operatorname{Hom}_{\mathfrak{g}}(\widetilde{V}, \widetilde{\mathcal{U}}) \quad \theta(\widetilde{V}) \subset \operatorname{Ann} \widetilde{M}_{\mathfrak{b}}(\lambda)$. 
Assume that Ann $\widetilde{M}_{\mathfrak{b}}(\lambda)$ is a centrally generated ideal. Then the above conditions imply the equality $\operatorname{Hom}_{\mathfrak{g}}(\widetilde{V}, \widetilde{\mathcal{U}})=\widetilde{\chi} \operatorname{Hom}_{\mathfrak{g}}(\widetilde{V}, \widetilde{\mathcal{U}})$ which yields

$$
\operatorname{det} \mathcal{J}(\mathfrak{b}, \widetilde{V}) \subseteq \mathcal{P}(\widetilde{\chi}) \operatorname{det} \mathcal{J}(\mathfrak{b}, \widetilde{V}) .
$$

Since $\operatorname{det} \mathcal{J}(\mathfrak{b}, \widetilde{V})$ is a non-zero subset of $\mathcal{S}(\mathfrak{h})$ and $\mathcal{P}(\widetilde{\chi})$ is a subset of $\mathcal{S}(\mathfrak{h})$ which does not contain non-zero scalars, the last inclusion is impossible. Thus Ann $\widetilde{M}_{\mathfrak{b}}(\lambda)$ is not a centrally generated ideal.

7.3.3. Retain the notation of 6.1.2. There exists $z \in \mathfrak{h} \operatorname{such}$ that ad $z$ acts by zero on $\mathfrak{g}_{0}$ and by identity (resp., minus identity) on $\mathfrak{g}_{1}$ (resp., $\mathfrak{g}_{-1}$ ). Recall that $\rho_{1}=\frac{1}{2} \sum_{\beta \in \Delta_{1}^{+}} \beta$ and so $2 \rho_{1}(z)=\operatorname{dim} \mathfrak{g}_{1}$.

7.3.4. Lemma. There exists $\nu \in \mathfrak{h}^{*}$ such that

$$
\begin{aligned}
& \text { a) } \operatorname{Hom}_{\mathfrak{g}}(\widetilde{V}(\nu), \widetilde{\mathcal{U}}) \neq 0, \\
& \text { b) } \widetilde{V}(\nu) \text { is typical, } \\
& \text { c } \left.^{\prime}\right) \quad z(\nu)=\operatorname{dim} \mathfrak{g}_{1} .
\end{aligned}
$$

Proof. Recall that condition b) is equivalent to the inequality $t(\nu) \neq 0$ where $t=$ $\prod_{\beta \in \Delta_{1}^{+}}\left(\beta^{\vee}+(\rho, \beta)\right)$. Observe that $z \neq \beta^{\vee}$ for any $\beta \in \Delta_{1}^{+}$, since $\beta^{\vee}(\beta)=0 \neq z(\beta)$. Thus the restriction of the polynomial $t$ on the hyperplane

$$
S:=\left\{\mu \in \mathfrak{h}^{*} \mid z(\mu)=\operatorname{dim} \mathfrak{g}_{1},(\mathcal{Z}(\mathfrak{g}) \cap \mathfrak{h})(\mu)=0\right\}
$$

is a non-zero polynomial (one has $\mathcal{Z}(\mathfrak{g}) \cap \mathfrak{h}=0$ apart from the case $\mathfrak{g}=\mathfrak{g l}(m, n)$ ).

Consider the set

$$
X:=\left\{\mu \in \mathfrak{h}^{*} \mid \operatorname{Hom}_{\mathfrak{g}_{0}}\left(V(\mu), \mathcal{U}\left(\mathfrak{g}_{0}\right)\right) \neq 0\right\} .
$$

It is easy to see that $X$ is a Zariski dense subset of the hyperplane

$$
\left\{\mu \in \mathfrak{h}^{*} \mid z(\mu)=0,(\mathcal{Z}(\mathfrak{g}) \cap \mathfrak{h})(\mu)=0\right\}=S-2 \rho_{1} .
$$

Thus $t\left(\nu^{\prime}+2 \rho_{1}\right) \neq 0$ for a certain $\nu^{\prime} \in X$. Hence $\nu:=\nu^{\prime}+2 \rho_{1}$ fulfills conditions b) and $\left.\mathrm{c}^{\prime}\right)$. Let us show that $\nu$ satisfies a). Since $\nu^{\prime} \in X$, there exists a copy $V \cong V(\nu)$ inside $\mathcal{U}\left(\mathfrak{g}_{0}\right)$. Let $u$ be a highest weight vector of $V$. Then $u x_{I}$ is a non-zero primitive (that is, $\left(\mathfrak{n}_{0}^{+}+\mathfrak{g}_{1}\right)$-invariant) element of $\widetilde{\mathcal{U}}$ having weight $\nu$. Therefore the ad $\mathfrak{g}$-submodule generated by $u x_{I}$ is a finite-dimensional quotient of $\widetilde{M}(\nu)$. Since $t(\nu) \neq 0$, a unique finite-dimensional quotient of $\widetilde{M}(\nu)$ is isomorphic to $\widetilde{V}(\nu)$. Hence $\operatorname{Hom}_{\mathfrak{g}}(\widetilde{V}(\nu), \widetilde{\mathcal{U}}) \neq 0$.

7.3.5. Remark. Similarly there exists a typical simple module $\widetilde{V}$ with the lowest weight $\nu$ such that $\operatorname{Hom}_{\mathfrak{g}}(\widetilde{V}, \widetilde{\mathcal{U}}) \neq 0$ and $z(\nu)=-\operatorname{dim} \mathfrak{g}_{1}$.

7.3.6. Fix $\lambda \in \mathfrak{h}^{*}$ such that $\mathcal{P}_{\mathfrak{b}}\left(x_{I_{+}} y_{I_{+}}\right)(\lambda)=0$. Set $\widetilde{\chi}:=\operatorname{Ann}_{\mathcal{Z}(\mathfrak{g})} \widetilde{M}(\lambda)$ and assume that Ann $\widetilde{M}(\lambda)=\widetilde{\mathcal{U}} \widetilde{\chi}$.

Fix $\nu$ satisfying conditions a) $-\mathrm{c}^{\prime}$ ) of Lemma 7.3.4. We claim that $\nu$ fulfills condition c) of 7.3 .2

Indeed, let $v$ be a highest weight vector of $\widetilde{V}:=\widetilde{V}(\nu)$. Take any $\theta \in \operatorname{Hom}_{\mathfrak{g}}(\widetilde{V}, \widetilde{\mathcal{U}})$. By assumption $\left.\mathrm{c}^{\prime}\right) z v=\left(\operatorname{dim} \mathfrak{g}_{1}\right) v$, that is, $[z, \theta(v)]=\left(\operatorname{dim} \mathfrak{g}_{1}\right) \theta(v)$. Therefore $\theta(v)$ has degree $\operatorname{dim} \mathfrak{g}_{1}$, that is, $\theta(v) \in \mathcal{U}\left(\mathfrak{g}_{0}\right) x_{I}$. Lemma 6.3.4 asserts that $x_{I} \widetilde{M}_{\mathfrak{b}}(\lambda)=0$ and so $\theta(v) \in \widetilde{M}_{\mathfrak{b}}(\lambda)=0$. Since Ann $\widetilde{M}_{\mathfrak{b}}(\lambda)$ is ad $\mathfrak{g}$-stable, it contains $\theta(\widetilde{V})$.

By the assumption, Ann $\widetilde{M}(\lambda)=\widetilde{\mathcal{U}} \widetilde{\chi}$ and so the element $\theta(v)$ can be written in a form $\theta(v)=\sum z_{i} u_{i}$ where all $z_{i}$ belong to $\widetilde{\chi}$. Without loss of generality we can 
assume that each element $u_{i}$ has the same weight and the same central character as $\theta(v)$ with respect to the adjoint action of $\mathcal{Z}(\mathfrak{g}) \subset \widetilde{\mathcal{U}}$ on $\widetilde{\mathcal{U}}$. Since $\widetilde{V}$ is typical, $\widetilde{V}$ is a unique, up to isomorphism, cyclic module in $\mathcal{F}$ in with this central character. Therefore ad $\mathfrak{g}$-span of each $u_{i}$ is isomorphic to $\widetilde{V}$, that is, $u_{i} \in \theta_{i}(\widetilde{V})$ for certain $\theta_{i} \in \operatorname{Hom}_{\mathfrak{g}}(\widetilde{V}, \widetilde{\mathcal{U}})$. Since $u_{i}$ has weight $\nu$ and $v$ spans $\left.\widetilde{V}\right|_{\nu}$, one can assume that $u_{i}=\theta_{i}(v)$. Then $\theta(v)=\sum z_{i} \theta_{i}(v)$ and so $\theta=\sum z_{i} \theta_{i}$. Hence

$$
\operatorname{Hom}_{\mathfrak{g}}(\widetilde{V}, \widetilde{\mathcal{U}})=\widetilde{\chi} \operatorname{Hom}_{\mathfrak{g}}(\widetilde{V}, \widetilde{\mathcal{U}}) .
$$

Using the fact that $\mathcal{P}_{\mathfrak{b}}(z \theta)=\mathcal{P}_{\mathfrak{b}}(z) \mathcal{P}_{\mathfrak{b}}(\theta)$ for any $z \in \mathcal{Z}(\mathfrak{g}), \theta \in \operatorname{Hom}_{\mathfrak{g}}(\widetilde{V}(\nu), \widetilde{\mathcal{U}})$, one concludes $\operatorname{det} \mathcal{J}_{\mathfrak{b}}(\widetilde{V}(\nu)) \subseteq \mathcal{P}(\widetilde{\chi}) \operatorname{det} \mathcal{J}_{\mathfrak{b}}(\widetilde{V}(\nu))$. Since $\operatorname{det} \mathcal{J}_{\mathfrak{b}}(\widetilde{V}(\nu))$ is a non-zero subset of a polynomial algebra $\mathcal{S}(\mathfrak{h})$ and $\mathcal{P}(\widetilde{\chi})$ does not contain non-zero scalars, the last inclusion is impossible. Hence $\operatorname{Ann} \widetilde{M}(\lambda) \neq \widetilde{\mathcal{U}} \widetilde{\chi}$.

In the case $\mathcal{P}_{\mathfrak{b}}\left(y_{I_{-}} x_{I_{-}}\right)(\lambda)=0$ the proof is similar: one may choose $\widetilde{V}$ as in Remark 7.3 .5

Hence we prove that for any triangular decomposition the following theorem holds.

7.3.7. Theorem. The annihilator of a Verma module is a centrally generated ideal iff this module is typical.

7.4. Denote by Max $A$ the set of maximal ideals of an algebra $A$. In [PS1], the following theorem is proven.

7.4.1. Theorem. For any $\tilde{\chi} \in \operatorname{Max} \mathcal{Z}(\mathfrak{g})$ not containing $T^{2}$, there exists $\chi \in$ $\operatorname{Max} \mathcal{Z}\left(\mathfrak{g}_{0}\right)$ such that the algebra $\widetilde{\mathcal{U}} /(\widetilde{\mathcal{U}} \chi)$ is the matrix algebra over $\mathcal{U}\left(\mathfrak{g}_{0}\right) /\left(\mathcal{U}\left(\mathfrak{g}_{0}\right) \chi_{0}\right)$.

One can deduce from this theorem that the annihilator of a typical Verma module is centrally generated. On the other hand, one can deduce Theorem 7.4.1 from Proposition [7.1 and 7.3.1. In fact, take $\lambda \in \mathfrak{h}^{*}$ such that $\widetilde{\chi} \widetilde{M}(\lambda)=0$. Then $t(\lambda) \neq 0$ and so

$$
\begin{aligned}
\widetilde{\mathcal{U}} /(\widetilde{\mathcal{U}} \widetilde{\chi}) & =F(\widetilde{M}(\lambda), \widetilde{M}(\lambda))=F\left(M(\lambda) \otimes \Lambda \mathfrak{g}_{1}^{-}, M(\lambda) \otimes \Lambda \mathfrak{g}_{1}^{-}\right) \\
& =F(M(\lambda), M(\lambda)) \otimes \operatorname{End}\left(\Lambda \mathfrak{g}_{1}^{-}\right)=\mathcal{U}\left(\mathfrak{g}_{0}\right) /\left(\mathcal{U}\left(\mathfrak{g}_{0}\right) \chi\right) \otimes \operatorname{End}\left(\Lambda \mathfrak{g}_{1}^{-}\right)
\end{aligned}
$$

where $\chi:=\operatorname{Ann}_{\mathcal{Z}}\left(\mathfrak{g}_{0}\right) M(\lambda)$. Hence $\widetilde{\mathcal{U}} /(\widetilde{\mathcal{U}} \chi)$ is a matrix algebra over $\mathcal{U}\left(\mathfrak{g}_{0}\right) /\left(\mathcal{U}\left(\mathfrak{g}_{0}\right) \chi\right)$.

\section{PERfECt PAIRS}

In this section we find for each maximal ideal of $\mathcal{Z}(\mathfrak{g})$ not containing $T^{2}$ a perfect mate which is a maximal ideal in $\mathcal{Z}\left(\mathfrak{g}_{0}\right)$ possessing certain properties.

8.1. Denote by Max $A$ the set of maximal ideals of an algebra $A$ and by $A$-Mod the full category of left $A$-modules. For an $A$-module $N$ and $\chi \in \operatorname{Max} A$ set

$$
N_{\chi}:=\left\{v \in N \mid \chi^{r} v=0, \forall r>>0\right\}
$$

Call a maximal ideal of $\mathcal{Z}(\mathfrak{g})$ a $\mathfrak{g}$-central character. For $\tilde{\chi} \in \operatorname{Max} \mathcal{Z}(\mathfrak{g})$ (resp., $\left.\chi \in \operatorname{Max} \mathcal{Z}\left(\mathfrak{g}_{0}\right)\right)$ set

$$
\tilde{\mathcal{U}}_{\tilde{\chi}}:=\tilde{\mathcal{U}} /(\tilde{\mathcal{U}} \widetilde{\chi}), \quad \mathcal{U}_{\chi}:=\mathcal{U} /(\mathcal{U} \chi)
$$

We canonically identify the $\widetilde{\mathcal{U}}_{\tilde{\chi}}$-modules and the (non-graded) $\widetilde{\mathcal{U}}$-modules annihilated by $\tilde{\chi}$. 
8.1.1. Definition. Call $\widetilde{\chi} \in \operatorname{Max} \mathcal{Z}(\mathfrak{g})$ strongly typical if $T^{2} \notin \widetilde{\chi}$.

Throughout this section $\tilde{\chi}$ denotes a strongly typical central character.

8.1.2. For a fixed triangular decomposition set

$$
W(\widetilde{\chi}):=\left\{\lambda \in \mathfrak{h}^{*} \mid \widetilde{\chi} \widetilde{M}(\lambda)=0\right\} .
$$

Recall that $\mathcal{P}(\mathcal{Z}(\mathfrak{g})) \supset \mathcal{P}\left(T^{2}\right) \mathcal{S}(\mathfrak{h})^{W}$. and so $W(\widetilde{\chi})$ forms a single $W$.-orbit.

Remark that $\tilde{\chi}$ is strongly typical iff any $\lambda \in W(\widetilde{\chi})$ is strongly typical.

For any pair $(\widetilde{\chi}, \chi) \in \operatorname{Max} \mathcal{Z}(\mathfrak{g}) \times \operatorname{Max} \mathcal{Z}\left(\mathfrak{g}_{0}\right)$ there is a functor $\Psi_{\tilde{\chi}, \chi}: \widetilde{\mathcal{U}}_{\tilde{\chi}}$-Mod $\rightarrow$ $\mathcal{U}_{\chi}$-Mod given by $\widetilde{N} \mapsto \widetilde{N}_{\chi}$. One of our goals is to prove that any strongly typical $\mathfrak{g}$ central character $\widetilde{\chi}$ has a "perfect mate" $\chi \in \operatorname{Max} \mathcal{Z}\left(\mathfrak{g}_{0}\right)$ such that the above functor provides the equivalence of categories. For the basic classical Lie superalgebras of type I this is proven in [PS1]. In [G2], we will prove it for the basic classical Lie superalgebras of type II.

For the type I case, it turns out that for any $\lambda \in W(\widetilde{\chi}), \chi:=\operatorname{Ann}_{\mathcal{Z}\left(\mathfrak{g}_{0}\right)} M(\lambda)$ is a perfect mate for $\tilde{\chi}$ (provided that $\tilde{\chi}$ is strongly typical) - see also 7.4. This does not hold for the type II case.

It is easy to see that if the functor $\Psi_{\tilde{\chi}, \chi}$ provides the equivalence of the categories, then $\chi$ has, at least, the following properties: for a Verma $\widetilde{\mathcal{U}}_{\tilde{\chi}^{-} \text {-module } \widetilde{M}}$ the $\mathfrak{g}_{0^{-}}$ module $\widetilde{M}_{\chi}$ is a Verma $\mathfrak{g}_{0}$-module and for any $\widetilde{\mathcal{U}}_{\tilde{\chi}}$-module $\widetilde{N}$ the $\mathfrak{g}_{0}$-module $\widetilde{N}_{\chi}$ is non-zero. We call $\chi$ a mate of $\tilde{\chi}$ if it possesses the first property and a perfect mate if it possesses both properties. As we shall show in [G2], these two properties really ensure the equivalence of categories $\widetilde{\mathcal{U}}_{\tilde{\chi}}$-Mod and $\mathcal{U}_{\chi}$-Mod provided that $\tilde{\chi}$ is strongly typical.

A pair $(\widetilde{\chi}, \chi) \in \operatorname{Max} \mathcal{Z}(\mathfrak{g}) \times \operatorname{Max} \mathcal{Z}\left(\mathfrak{g}_{0}\right)$ is called a perfect pair if $\chi$ is a perfect mate for $\tilde{\chi}$.

The goal of this section is to find a perfect mate for any strongly typical $\tilde{\chi}$. This is done in the following way. Lemma 8.3.4 gives a combinatorial criterion on $\chi$ to be a perfect mate for $\tilde{\chi}$. In 8.5 we fix, for each basic classical Lie superalgebra of type II, a triangular decomposition, in terms of which we will describe a perfect mate $\chi$ for $\tilde{\chi}$. In 8.6 we consider $\tilde{\chi}$ satisfying a certain genericity condition. For such a "generic" $\tilde{\chi}$ we show that for a suitable $\lambda \in W(\widetilde{\chi})$ the $\mathfrak{g}_{0}$-central character of $M(\lambda)$ is a perfect mate for $\tilde{\chi}$. The case when $\tilde{\chi}$ is not generic is treated in 8.7 It turns out that for $\mathfrak{g}$ of the types $B(m, n), G(3)$ any strongly typical $\tilde{\chi}$ is "generic". The remaining basic classical Lie superalgebras of type II are treated case by case.

\subsection{Notation.}

8.2.1. We say that a $\widetilde{\mathcal{U}}$-module $\widetilde{N}$ has a finite support $\operatorname{supp}_{\mathcal{Z}(\mathfrak{g})} \widetilde{N}=\left\{\widetilde{\chi}_{1}, \ldots, \widetilde{\chi}_{k}\right\}$ if for any $v \in \widetilde{N}$ there exist $r_{1}, \ldots, r_{k} \in \mathbb{N}^{+}$such that $\prod_{i} \widetilde{\chi}_{i}^{r_{i}} v=0$. In this case,

$$
\widetilde{N}=\bigoplus_{i} \tilde{N}_{\tilde{\chi}_{i}}
$$

and each $\widetilde{N}_{\tilde{\chi}_{i}}$ is canonically isomorphic to the localization of the module $\widetilde{N}$ by the maximal ideal $\tilde{\chi}_{i}$. If $\widetilde{N}$ has a finite support and $0 \rightarrow \widetilde{N}^{\prime} \rightarrow \widetilde{N} \rightarrow \widetilde{N}^{\prime \prime} \rightarrow 0$ is an exact sequence, then, for any $\tilde{\chi}^{\prime} \in \operatorname{Max} \mathcal{Z}(\mathfrak{g})$, the sequence $0 \rightarrow \widetilde{N}_{\tilde{\chi}^{\prime}}^{\prime} \rightarrow \widetilde{N}_{\widetilde{\chi}^{\prime}} \rightarrow \widetilde{N}_{\widetilde{\chi}^{\prime}}^{\prime \prime} \rightarrow 0$ is also exact.

For a $\mathfrak{g}$-module $\widetilde{N}$ and a maximal ideal $\widetilde{\chi}^{\prime} \in \operatorname{Max} \mathcal{Z}(\mathfrak{g})$ define $\widetilde{\chi}^{\prime}$-depth of $\widetilde{N}$ to be a minimal $r \in \mathbb{N} \cup \infty$ such that $\left(\widetilde{\chi}^{\prime}\right)^{r} \widetilde{N}_{\tilde{\chi}^{\prime}}=0$. 
We adopt similar notation for $\mathcal{Z}\left(\mathfrak{g}_{0}\right)$ and $\mathcal{U}$-modules.

8.2.2. Denote by $\Gamma$ the set of subsets of $\Delta_{1}^{+}$. For $\gamma \subseteq \Delta_{1}^{+}$set

$$
|\gamma|:=\sum_{\beta \in \gamma} \beta .
$$

Define the action of the Weyl group $W$ on $\Gamma$ by setting

$$
w_{*} \gamma:=w\left(\gamma \cup-\left(\Delta_{1}^{+} \backslash \gamma\right)\right) \cap \Delta_{1}^{+} \text {. }
$$

Then

$$
\left|w_{*} \gamma\right|=w\left(|\gamma|-\rho_{1}\right)+\rho_{1}
$$

8.2.3. As a $\mathfrak{g}_{0}$-module, a Verma module $\widetilde{M}(\lambda)$ has a filtration $0=N_{0} \subset N_{1} \subset$ $\cdots \subset N_{r}=\widetilde{M}(\lambda)$ such that the set of factors $N_{i+1} / N_{i}$ coincides with the multiset $\{M(\lambda-\gamma): \gamma \in \Gamma\}$ - see M1], 3.2.

It is easy to check that for any $w \in W, \gamma \in \Gamma$

$$
w \cdot \lambda-\left|w_{*} \gamma\right|+\rho_{0}=w\left(\lambda-|\gamma|+\rho_{0}\right) .
$$

Therefore the $\mathfrak{g}_{0}$-central characters of $M\left(w \cdot \lambda-\left|w_{*} \gamma\right|\right)$ and $M(\lambda-|\gamma|)$ coincide. Thus for fixed $\widetilde{\chi}$ the multiset of $\mathfrak{g}_{0}$-central characters of $\{M(\lambda-\gamma): \gamma \in \Gamma\}$ does not depend on the choice of $\lambda \in W(\widetilde{\chi})$.

8.2.4. Definition. Call $\chi \in \operatorname{Max} \mathcal{Z}\left(\mathfrak{g}_{0}\right)$ a mate for $\tilde{\chi} \in \operatorname{Max} \mathcal{Z}(\mathfrak{g})$ if for some $\widetilde{M}(\lambda)$ satisfying $\widetilde{\chi} \widetilde{M}(\lambda)=0$, the $\mathfrak{g}_{0}$-module $\widetilde{M}(\lambda)_{\chi}$ is isomorphic to a Verma module $M(\lambda-|\gamma|)$ for some $\gamma \in \Gamma$.

One can easily deduce from 8.2 .3 that if $\chi$ is a mate for $\tilde{\chi}$, then for any $\widetilde{M}(\lambda)$ satisfying $\widetilde{\chi} \widetilde{M}(\lambda)=0$, the $\mathfrak{g}_{0}$-module $\widetilde{M}(\lambda)_{\chi}$ is isomorphic to a Verma module $M(\lambda-|\gamma|)$ for some $\gamma \in \Gamma$. In particular, if $\chi$ is a mate for $\tilde{\chi}$, then for any $\widetilde{M}$ satisfying $\widetilde{\chi} \widetilde{M}=0$, the $\chi$-depth of $\widetilde{M}$ is equal to 1 .

8.2.5. Definition. Let us call $\chi \in \operatorname{Max} \mathcal{Z}\left(\mathfrak{g}_{0}\right)$ a perfect mate for $\tilde{\chi} \in \operatorname{Max} \mathcal{Z}(\mathfrak{g})$ if it is a mate for $\tilde{\chi}$ and for any non-zero $\widetilde{\mathcal{U}}_{\tilde{\chi}}$-module $\widetilde{N}$ one has $\tilde{N}_{\chi} \neq 0$.

Definition 8.2 .4 is given in terms of the category $\widetilde{\mathcal{O}}$. However, 2.7 implies that if for some triangular decomposition $\chi$ is a mate for $\tilde{\chi}$, then it is a mate also with respect to all other choices of triangular decomposition.

8.3. Throughout this subsection a strongly typical $\mathfrak{g}$-central character $\tilde{\chi}$ is assumed to be fixed. Our first goal is to reformulate Definition 8.2.5 in terms of category $\widetilde{\mathcal{O}}$.

8.3.1. Lemma. There exist $\chi_{1}, \ldots, \chi_{k} \in \operatorname{Max} \mathcal{Z}\left(\mathfrak{g}_{0}\right)$ and $r_{1}, \ldots, r_{k} \in \mathbb{N}^{+}$such that for any $\widetilde{\mathcal{U}}_{\tilde{\chi}}$-module $\tilde{N}$ one has

$$
\tilde{N}=\bigoplus_{1}^{k} \tilde{N}_{\chi_{i}}
$$

and $\chi^{r_{i}} \tilde{N}_{\chi_{i}}=0$ 
Proof. Theorem 2.5 of [M1] implies that for any $x \in \mathcal{Z}\left(\mathfrak{g}_{0}\right)$ there exist $z_{0}, \ldots, z_{l} \in$ $\mathcal{Z}(\mathfrak{g})$ (where $l=\# \Gamma$ ) such that $\sum_{0}^{l} z_{i} x^{i}=0$ and $z_{l}=T^{2}$ (notice that 2.1 of [M1] contains a misprint; to correct it one has to substitute $g$ by $g^{2}$ in 2.1 and in Theorem 2.5). Therefore for any $x \in \mathcal{Z}\left(\mathfrak{g}_{0}\right)$ there exist $c_{0}, \ldots, c_{l-1} \in \mathbb{C}$ such that $x^{l}+\sum_{0}^{l-1} c_{i} x^{i} \in\left(\mathcal{Z}\left(\mathfrak{g}_{0}\right) \cap \widetilde{\mathcal{U}} \widetilde{\chi}\right)$. Consequently, the ideal $\left(\mathcal{Z}\left(\mathfrak{g}_{0}\right) \cap \widetilde{\mathcal{U}} \widetilde{\chi}\right)$ has a finite codimension in $\mathcal{Z}\left(\mathfrak{g}_{0}\right)$ and so there exist $\chi_{1}, \ldots, \chi_{k} \in \operatorname{Max} \mathcal{Z}\left(\mathfrak{g}_{0}\right)$ and $r_{1}, \ldots, r_{k} \in \mathbb{N}^{+}$ such that

$$
\left(\mathcal{Z}\left(\mathfrak{g}_{0}\right) \cap \widetilde{\mathcal{U}} \widetilde{\chi}\right) \supseteq \prod_{i=1}^{k} \chi_{i}^{r_{i}} .
$$

Then, for any $\widetilde{\mathcal{U}}_{\tilde{\chi}}$-module $\widetilde{N}$

$$
\prod_{i=1}^{k} \chi_{i}^{r_{i}} \subseteq \operatorname{Ann}_{\mathcal{Z}\left(\mathfrak{g}_{0}\right)} \tilde{N}
$$

The assertion follows.

8.3.2. Corollary. If $\chi$ is a perfect mate for $\tilde{\chi}$, then

$$
\widetilde{N}=\tilde{\mathcal{U}} \tilde{N}_{\chi}
$$

for any $\widetilde{\mathcal{U}}_{\tilde{\chi}}$-module $\tilde{N}$.

Proof. Since $\operatorname{supp}_{\mathcal{Z}\left(\mathfrak{g}_{0}\right)} \tilde{N}$ is finite, one has $\left(\widetilde{N} /\left(\widetilde{\mathcal{U}} \widetilde{N}_{\chi}\right)\right)_{\chi}=0$. Hence $\widetilde{N} /\left(\widetilde{\mathcal{U}} \widetilde{N}_{\chi}\right)=0$ because $\chi$ is a perfect mate for $\tilde{\chi}$.

8.3.3. Lemma. If $\chi \in \operatorname{Max} \mathcal{Z}\left(\mathfrak{g}_{0}\right)$ is a mate for $\tilde{\chi} \in \operatorname{Max} \mathcal{Z}(\mathfrak{g})$ and $\tilde{V}(\lambda)_{\chi} \neq 0$ for all $\lambda \in W(\widetilde{\chi})$, then $\chi$ is a perfect mate.

Proof. Suppose that $\widetilde{V}(\lambda)_{\chi} \neq 0$ for all $\lambda \in W(\widetilde{\chi})$. One has to verify that $\widetilde{N}_{\chi} \neq 0$ for any non-zero $\widetilde{\mathcal{U}}_{\tilde{\chi}}$-module $\widetilde{N}$. Since $\widetilde{\mathcal{U}}$ is Noetherian, $\widetilde{N}$ has a simple subquotient $\tilde{N}^{\prime}$. Lemma 8.3 .1 implies that $\widetilde{N}_{\chi} \neq 0$ provided $\tilde{N}_{\chi}^{\prime} \neq 0$. Hence it is enough to check that $\widetilde{N}_{\chi} \neq 0$ for any simple $\widetilde{\mathcal{U}}_{\tilde{\chi}}$-module $\widetilde{N}$.

Take a simple $\widetilde{\mathcal{U}}_{\tilde{\chi}}$-module $\widetilde{N}$. The ideal Ann $\widetilde{N}$ is primitive and so, according to [M2], Ann $\widetilde{N}=$ Ann $\widetilde{V}$ where $\widetilde{V}$ is a simple highest weight module. Obviously, $\widetilde{\chi} \widetilde{V}=0$ and so, by our assumption, $\widetilde{V}_{\chi} \neq 0$. As a $\mathfrak{g}_{0}$-module, $\widetilde{V}$ has a finite length. Therefore

$$
\operatorname{Ann}_{\mathcal{Z}\left(\mathfrak{g}_{0}\right)} \tilde{N}=\operatorname{Ann}_{\mathcal{Z}\left(\mathfrak{g}_{0}\right)} \widetilde{V}=\chi^{r_{1}} \chi_{2}^{r_{2}} \cdots \chi_{k}^{r_{k}}
$$

where $\left\{\chi, \chi_{2}, \ldots, \chi_{k}\right\}=\operatorname{supp}_{\mathcal{Z}\left(\mathfrak{g}_{0}\right)} \tilde{V}, r_{1}, \ldots, r_{k} \in \mathbb{N}^{+}$. Then $\widetilde{N}^{\prime}:=\chi_{2}^{r_{2}} \cdots \chi_{k}^{r_{k}} \widetilde{N} \neq$ 0 and $\chi^{r_{1}} \widetilde{N}^{\prime}=0$. Hence $\widetilde{N}_{\chi} \neq 0$. The lemma is proved.

8.3.4. Lemma. Take $\lambda \in W(\widetilde{\chi}), \gamma \in \Gamma$ and set $\chi:=\operatorname{Ann}_{\mathcal{Z}\left(\mathfrak{g}_{0}\right)} M(\lambda-|\gamma|)$.

(i) $\forall \gamma^{\prime} \in \Gamma \backslash\{\gamma\} \quad\left(\lambda-\left|\gamma^{\prime}\right|+\rho_{0}\right) \notin W\left(\lambda-|\gamma|+\rho_{0}\right) \quad \Longleftrightarrow \quad \chi$ is a mate for $\tilde{\chi}$.

(ii) If $\chi$ is a mate for $\tilde{\chi}$ and $\operatorname{Stab}_{W}\left(\lambda-|\gamma|+\rho_{0}\right) \subseteq \operatorname{Stab}_{W}(\lambda+\rho)$, then $\chi$ is a perfect mate.

Proof. Equivalence (i) follows from 8.2 .3 . 
For (ii) recall Lemma 8.3.3. Suppose that $\chi$ is a mate for $\tilde{\chi}$ but it is not a perfect mate. Then $\widetilde{M}(\lambda)_{\chi}=M(\lambda-|\gamma|)$ and $\widetilde{V}(w \cdot \lambda)_{\chi}=0$ for some $w \in W$. Equality (7) implies that for any $y \in W$

$$
\widetilde{M}(y \cdot \lambda)_{\chi}=M\left(y \cdot \lambda-\left|y_{*} \gamma\right|\right) .
$$

Therefore $\tilde{V}(y \cdot \lambda)_{\chi}$ is a quotient of $M\left(y \cdot \lambda-\left|y_{*} \gamma\right|\right)$.

The module $\widetilde{V}(w \cdot \lambda)$ is a homomorphic image of $\widetilde{M}:=\widetilde{M}(w \cdot \lambda)$. Denote the kernel of this homomorphism by $\tilde{N}$. The module $\widetilde{N}$ has a finite length and the factors of its Jordan-Gölder series have the form $\widetilde{V}(\mu)$ for some $\mu \in W \cdot \lambda$ satisfying $\mu<w \cdot \lambda$. Since $0=\tilde{V}(w \cdot \lambda)_{\chi}=(\widetilde{M} / \widetilde{N})_{\chi}$, one concludes that the $\mathfrak{g}_{0}$-module $\widetilde{N}_{\chi}=\widetilde{M}_{\chi}=M\left(w \cdot \lambda-\left|w_{*} \gamma\right|\right)$ has a finite filtration such that each factor of this filtration is a quotient of $M\left(y \cdot \lambda-\left|y_{*} \gamma\right|\right)$ for some $y \in W$ satisfying $y \cdot \lambda<w \cdot \lambda$. Therefore

$$
w \cdot \lambda-\left|w_{*} \gamma\right|=y \cdot \lambda-\left|y_{*} \gamma\right|
$$

for some $y \in W$ satisfying $y . \lambda<w . \lambda$. By (7), the above equality is equivalent to the condition $y^{-1} w \in \operatorname{Stab}_{W}\left(\lambda+\rho_{0}-|\gamma|\right)$. However, $y \cdot \lambda<w \cdot \lambda$ implies $y^{-1} w \notin$ $\operatorname{Stab}_{W}(\lambda+\rho)$. Thus $\operatorname{Stab}_{W}\left(\lambda+\rho_{0}-|\gamma|\right) \nsubseteq \operatorname{Stab}_{W}(\lambda+\rho)$ as required.

8.4. Throughout this subsection a strongly typical $\mathfrak{g}$-central character $\tilde{\chi}$ is assumed to be fixed.

8.4.1. Right version. One may repeat the above reasonings for the right $\mathfrak{g}$-modules. Suppose now that $(\widetilde{\chi}, \chi)$ is a perfect pair in the "left sense". We claim that it is a perfect pair in the "right sense".

Indeed, the superantiautomorphism $\sigma$ (see 2.3) provides the duality between the left and the right $\widetilde{\mathcal{U}}$-modules given by $N \mapsto N^{\sigma}$ :

$$
v^{\sigma} . a:=(-1)^{d(a) d(v)} \sigma(a) \cdot v, \quad a \in \tilde{\mathcal{U}}, v \in N .
$$

By 2.3.4, $\sigma$ stabilizes the elements of $\mathcal{Z}(\mathfrak{g})$; thus the restriction of the above duality gives the duality between the left and the right $\widetilde{\mathcal{U}}_{\tilde{\chi}}$-modules. Since $\sigma$ also stabilizes the elements of $\mathcal{Z}\left(\mathfrak{g}_{0}\right)$, in order to show that $(\widetilde{\chi}, \chi)$ is a perfect pair in the "right sense", it is enough to check that $\chi$ is mate for $\tilde{\chi}$ in the "right sense".

Take a minimal element $\lambda \in W(\widetilde{\chi})$. Then $\widetilde{M}:=\widetilde{M}(\lambda)$ is a simple Verma module and $\widetilde{M^{\sigma}}$ is a right simple module which is a Verma module with respect to a suitable triangular decomposition of $\mathfrak{g}$. Since $\chi$ is a mate for $\widetilde{\chi}, \widetilde{M}_{\chi}$ is a Verma $\mathfrak{g}_{0}$-module, say $M$. Then

$$
\chi^{\widetilde{M}^{\sigma}}:=\left\{v \in \widetilde{M}^{\sigma} \mid v \cdot \chi^{r}=0, \quad r>>0\right\}=\left(\widetilde{M}_{\chi}\right)^{\sigma}=M^{\sigma}
$$

since $\sigma$ stabilizes the elements of $\mathcal{Z}\left(\mathfrak{g}_{0}\right)$. Therefore ${ }_{\chi} \widetilde{M^{\sigma}}$ is a (right) $\mathfrak{g}_{0}$-submodule of a (right) Verma $\mathfrak{g}$-module and, at the same time, it is dual to a Verma $\mathfrak{g}_{0}$-module. Since a Verma $\mathfrak{g}$-module is $\mathcal{U}\left(\mathfrak{n}_{0}^{-}\right)$-torsion-free, ${ }_{\chi} \widetilde{M}^{\sigma}$ is a (right) Verma $\mathfrak{g}_{0}$-module. Hence $\chi$ is a mate for $\tilde{\chi}$ in the "right sense".

8.4.2. Let $\chi$ be a perfect mate for $\tilde{\chi}$, and let $L$ be a non-zero $\tilde{\mathcal{U}}$-bimodule such that $\tilde{\chi} \cdot L=L \cdot \tilde{\chi}=0$. Let us show that

$$
{ }_{\chi} L_{\chi}:=\left\{v \in L \mid \chi^{r} \cdot v=v \cdot \chi^{r}=0, \quad r>>0\right\}
$$

is non-zero. 
Indeed, according to Lemma 8.3.1, there exist pair-wise distinct $\mathfrak{g}_{0}$-central characters $\chi_{1}, \ldots, \chi_{k}$ and positive integers $r_{1}, \ldots, r_{k}$ such that $\prod_{1}^{k} \chi_{i}^{r_{i}} . L=0$. Recall that $L_{\chi} \neq 0$ since $(\tilde{\chi}, \chi)$ is a perfect pair. Thus $\chi=\chi_{i}$ for a certain $i$; we can assume that $\chi=\chi_{1}$. One has $\chi_{1}^{r_{1}}+\prod_{2}^{k} \chi_{i}^{r_{i}}=\mathcal{Z}\left(\mathfrak{g}_{0}\right)$ since $\chi_{1}, \ldots, \chi_{k} \in \operatorname{Max} \mathcal{Z}\left(\mathfrak{g}_{0}\right)$ are pair-wise distinct. Therefore

$$
0 \neq L_{\chi}=\prod_{2}^{k} \chi_{i}^{r_{i}} . L
$$

and it is a right submodule of $L$. Clearly

$$
{ }_{\chi} L_{\chi}=\left\{v \in L_{\chi} \mid v \cdot \chi^{r}=0, \quad r>>0\right\} .
$$

Using 8.4.1, one obtains ${ }_{\chi} L_{\chi} \neq 0$ as required.

8.5. By 8.2.5 to prove the existence of a perfect mate for a strongly typical $\mathfrak{g}$ central character, one can choose any triangular decomposition. Below we describe, for each basic classical Lie superalgebra of type II, a triangular decomposition we choose for the proof. We use notation of Kac- see K1, 2.5.4, for all cases except $D(1,2, \alpha)$ where we use $\delta, \epsilon_{1}, \epsilon_{2}$ instead $\epsilon_{1}, \epsilon_{2}, \epsilon_{3}$. The chosen triangular decomposition always corresponds to the first "simplest system of simple roots" in $\mathrm{K} 1$.

$$
\begin{aligned}
B(m, n): & \mathfrak{g}_{0}=\mathfrak{s o}(2 m+1) \times \mathfrak{s p}(2 n), \\
& \pi=\left\{\delta_{1}-\delta_{2}, \ldots, \delta_{n}-\epsilon_{1}, \epsilon_{1}-\epsilon_{2}, \ldots, \epsilon_{m-1}-\epsilon_{m}, \epsilon_{m}\right\}, \\
& \Delta_{0}^{+}=\left\{\delta_{i} \pm \delta_{j} ; 2 \delta_{i}\right\}_{1 \leq i<j \leq n} \cup\left\{\epsilon_{i} \pm \epsilon_{j}, ; \epsilon_{i}\right\}_{1 \leq i<j \leq m}, \\
& \Delta_{1}^{+}=\left\{\delta_{i} ; \delta_{i} \pm \epsilon_{j}\right\}_{1 \leq i \leq n, 1 \leq j \leq m}, \\
& \rho_{1}=\left(m+\frac{1}{2}\right)\left(\delta_{1}+\delta_{2}+\cdots+\delta_{n}\right)
\end{aligned}
$$

where $\left\{\delta_{1}-\delta_{2}, \ldots, 2 \delta_{n}\right\}$ is a system of simple roots of $\mathfrak{s p}(2 n)$ and $\left\{\epsilon_{1}-\epsilon_{2}, \ldots, \epsilon_{m-1}-\right.$ $\left.\epsilon_{m}, \epsilon_{m}\right\}$ is a system of simple roots of $\mathfrak{s o}(2 m+1)$.

$$
\begin{aligned}
D(m, n): & \mathfrak{g}_{0}=\mathfrak{s o}(2 m) \times \mathfrak{s p}(2 n), \\
& \pi=\left\{\delta_{1}-\delta_{2}, \ldots, \delta_{n}-\epsilon_{1}, \epsilon_{1}-\epsilon_{2}, \ldots, \epsilon_{m-1}-\epsilon_{m}, \epsilon_{m-1}+\epsilon_{m}\right\}, \\
& \Delta_{0}^{+}=\left\{\delta_{i} \pm \delta_{j}, 2 \delta_{i}\right\}_{1 \leq i<j \leq n} \cup\left\{\epsilon_{i} \pm \epsilon_{j}\right\}_{1 \leq i<j \leq m}, \\
& \Delta_{1}^{+}=\left\{\delta_{i} \pm \epsilon_{j}\right\}_{1 \leq i \leq n, 1 \leq j \leq m}, \\
& \rho_{1}=m\left(\delta_{1}+\delta_{2}+\cdots+\delta_{n}\right)
\end{aligned}
$$

where $\left\{\delta_{1}-\delta_{2}, \ldots, 2 \delta_{n}\right\}$ is a system of simple roots of $\mathfrak{s p}(2 n)$ and $\left\{\epsilon_{1}-\epsilon_{2}, \ldots, \epsilon_{m-1}-\right.$ $\left.\epsilon_{m}, \epsilon_{m-1}+\epsilon_{m}\right\}$ is a system of simple roots of $\mathfrak{s o}(2 m)$.

$$
\begin{aligned}
D(2,1, \alpha): \quad & \mathfrak{g}_{0}=\mathfrak{s l}(2) \times \mathfrak{s l}(2) \times \mathfrak{s l}(2), \\
& \pi=\left\{\delta_{1}+\epsilon_{1}+\epsilon_{2},-2 \epsilon_{1},-2 \epsilon_{2}\right\}, \\
& \Delta_{0}^{+}=\left\{2 \delta_{1} ;-2 \epsilon_{1},-2 \epsilon_{2}\right\}, \\
& \Delta_{1}^{+}=\left\{\delta_{1} \pm \epsilon_{1} \pm \epsilon_{2}\right\} \\
& \rho_{1}=2 \delta_{1} . \\
& \\
& \mathfrak{g}_{0}=\mathfrak{s l}(2) \times \mathfrak{s o}(7), \\
& \pi=\left\{\frac{1}{2}\left(\epsilon_{1}+\epsilon_{2}+\epsilon_{3}+\delta_{1}\right),-\epsilon_{1}, \epsilon_{1}-\epsilon_{2}, \epsilon_{2}-\epsilon_{3}\right\}, \\
& \Delta_{0}^{+}=\left\{\delta_{1}\right\} \cup\left\{-\epsilon_{i}, 1 \leq i \leq 3 ; \pm \epsilon_{i}-\epsilon_{j}, 1 \leq i<j \leq 3\right\}, \\
& \Delta_{1}^{+}=\left\{\frac{1}{2}\left(\delta_{1} \pm \epsilon_{1} \pm \epsilon_{2} \pm \epsilon_{3}\right)\right\} \\
& \rho_{1}=2 \delta_{1}
\end{aligned}
$$


where $\delta_{1}$ is a simple root of $\mathfrak{s l}(2)$ and $\left\{-\epsilon_{1}, \epsilon_{1}-\epsilon_{2}, \epsilon_{2}-\epsilon_{3}\right\}$ is a system of simple roots of $\mathfrak{s o}(7)$.

$$
\begin{aligned}
G(3): & \mathfrak{g}_{0}=\mathfrak{s l}(2) \times G_{2}, \\
& \pi=\left\{\delta_{1}+\epsilon_{1}, \epsilon_{2}, \epsilon_{3}-\epsilon_{2}\right\}, \\
& \Delta_{0}^{+}=\left\{2 \delta_{1}\right\} \cup\left\{\epsilon_{2}, \epsilon_{3},-\epsilon_{1}, \epsilon_{3}-\epsilon_{2}, \epsilon_{3}-\epsilon_{1}, \epsilon_{2}-\epsilon_{1}\right\}, \\
& \Delta_{1}^{+}=\left\{\delta_{1} ; \delta_{1} \pm \epsilon_{i}, i=1,2,3\right\} \\
& \rho_{1}=\frac{7}{2} \delta_{1}
\end{aligned}
$$

where $\epsilon_{1}+\epsilon_{2}+\epsilon_{3}=0,\left\{\epsilon_{2}, \epsilon_{3}-\epsilon_{2}\right\}$ is a system of simple roots of $G(2)$ and $2 \delta_{1}$ is a simple root of $\mathfrak{s l}(2)$.

The restriction of the non-degenerate invariant bilinear form $(-,-)$ on $\mathfrak{g}_{0}$ is a non-degenerate invariant bilinear form. Thus

$$
\left(\delta_{i}, \delta_{i^{\prime}}\right)=\left(\delta_{i}, \epsilon_{j}\right)=0 \quad \forall i \neq i^{\prime}
$$

and

$$
\left(\epsilon_{j}, \epsilon_{j^{\prime}}\right)=0 \quad \forall j \neq j^{\prime}
$$

if $\mathfrak{g}$ is not of the type $G(3)$.

Until the end of this section $\mathfrak{g}$ will denote one (unless otherwise specified, an arbitrary one) of the basic classical Lie superalgebras of type II with the fixed triangular decomposition described above.

8.5.1. More notation. For all of the above root systems denote by $n$ the number of $\delta$ 's and by $m$ the number of $\epsilon$ 's (that is, $n=1$ for $D(1,2, \alpha), F(4), G(3)$ and $m=2$ for $D(1,2, \alpha), m=3$ for $F(4), G(3))$. Remark that for all of the above systems

$$
\rho_{1}=p \sum_{i=1}^{n} \delta_{i}
$$

for a certain scalar $p \in \frac{1}{2} \mathbb{N}^{+}$.

Denote by $W_{1}$ (resp., $W_{2}$ ) the subgroup of $W$ which acts on $\left\{\delta_{i}\right\}_{i=1}^{n}$ (resp., on $\left.\left\{\epsilon_{j}\right\}_{j=1}^{m}\right)$. Then $W=W_{1} \times W_{2}$. Remark that $W_{1}$ is always the group of signed permutations of $\left\{\delta_{i}\right\}_{i=1}^{n}$ : for $B(m, n)$ and $D(m, n)$ it is the Weyl group corresponding to $\mathfrak{s p}(2 n)$ and for $D(1,2, \alpha), F(4), G(3)$ it is the Weyl group corresponding to $\mathfrak{s l}(2)$.

For $\mu \in \mathfrak{h}^{*}$ write

$$
\mu=\sum_{i=1}^{n} k_{i} \delta_{i}+\sum_{j=1}^{m} l_{j} \epsilon_{j}
$$

and set $\mu_{\delta_{i}}:=k_{i}, \mu_{\epsilon_{j}}:=l_{j}$.

Say that $\tilde{\chi}$ is generic if there exists $\lambda \in W(\widetilde{\chi})$ such that $(\lambda+\rho)_{\delta_{i}} \neq 0$ for $i=1, \ldots, n$. Remark that $T^{2} \notin \tilde{\chi}$ implies that $\tilde{\chi}$ is generic if $\delta_{1} \in \Delta_{1}$, that is, in the cases $B(m, n)$ and $G(3)$.

Define a lexicographic order on $\mathbb{C}$ by setting $c_{1}>c_{2}$ if $\operatorname{Re} c_{1}>\operatorname{Re} c_{2}$ or $\operatorname{Re} c_{1}=$ $\operatorname{Re} c_{2}$ and $\operatorname{Im} c_{1}>\operatorname{Im} c_{2}$.

One can easily see that for any $\gamma \in \Gamma$ and $i=1, \ldots, n$ one has $0 \leq|\gamma|_{\sigma_{i}} \leq 2 p$ and, moreover, $\gamma=\emptyset$ iff $|\gamma|_{\sigma_{1}}=\ldots=|\gamma|_{\sigma_{n}}=0$. We will frequently use the following easy lemma. 
8.5.2. Lemma. Let $d ; a_{1}, \ldots, a_{s} ; r_{1}, \ldots, r_{s}$ be complex numbers such that $d$, $a_{1}, \ldots, a_{s}>0$ and $0 \leq r_{1}, \ldots, r_{s} \leq 2 d$. Suppose there exists a signed permutation $\sigma$ which maps $\left(a_{1}+d, \ldots, a_{s}+d\right)$ to $\left(a_{1}+d-r_{1}, \ldots, a_{s}+d-r_{s}\right)$. Then $\sigma$ is a usual (non-signed) permutation and $r_{1}=\cdots=r_{s}=0$.

Proof. The permutation $\sigma$ is non-signed because $a_{i}+a_{j}>0 \geq-2 d+r_{j}$ and so $a_{i}+d \neq-\left(a_{j}+d-r_{j}\right)$ for any indexes $i, j$. Since $\sigma$ is a usual permutation,

$$
\sum_{i}^{s}\left(a_{i}+d\right)=\sum_{1}^{s}\left(a_{i}+d-r_{i}\right)
$$

that is, $\sum r_{i}=0$. The inequalities $r_{1}, \ldots, r_{s} \geq 0$ imply the assertion.

8.6. Generic case. Fix a generic central character $\tilde{\chi}$. Since $W_{1}$ acts by signed permutation on $\left\{\delta_{i}\right\}_{i=1}^{n}$, there exists $\lambda \in W(\tilde{\chi})$ such that $(\lambda+\rho)_{\delta_{i}}>0$ for all $i=1, \ldots, n$. Fix such a $\lambda$ and let us show that the $\mathfrak{g}_{0}$-character of $M(\lambda)$ is a perfect mate for $\tilde{\chi}$.

To verify that the $\mathfrak{g}_{0}$-central character of $M(\lambda)$ is a mate for $\tilde{\chi}$, recall Lemma 8.3.4 (i). Suppose that

$$
\left(\lambda+\rho_{0}\right)=w\left(\lambda+\rho_{0}-|\gamma|\right)
$$

for some $\gamma \in \Gamma, w \in W$. Write

$$
\lambda+\rho=\sum_{1}^{n} k_{i} \delta_{i}+\sum_{1}^{m} l_{j} \epsilon_{j}, \quad|\gamma|=\sum_{1}^{n} s_{i} \delta_{i}+\sum_{1}^{m} r_{j} \epsilon_{j} .
$$

Recall that $k_{i}>0$ and $0 \leq s_{i} \leq 2 p$ for $i=1, \ldots, n$. One has

$$
\lambda+\rho_{0}=\lambda+\rho+\rho_{1}=\sum\left(k_{i}+p\right) \delta_{i}+\sum l_{j} \epsilon_{j}
$$

and

$$
\lambda+\rho_{0}-|\gamma|=\sum\left(k_{i}+p-s_{i}\right) \delta_{i}+\sum\left(l_{j}-r_{j}\right) \epsilon_{j}
$$

Write $w=w_{1} w_{2}$ where $w_{1} \in W_{1}, w_{2} \in W_{2}$. Then

$$
\sum\left(k_{i}+p\right) \delta_{i}=w_{1}\left(\sum\left(k_{i}+p-s_{i}\right) \delta_{i}\right)
$$

By Lemma 8.5.2, this implies $s_{1}=\cdots=s_{n}=0$ and thus $\gamma=\emptyset$. Hence the $\mathfrak{g}_{0}$-central character of $M(\lambda)$ is a mate for $\tilde{\chi}$.

One has

$$
\begin{aligned}
\operatorname{Stab}_{W}\left(\lambda+\rho_{0}\right) & =\operatorname{Stab}_{W}\left(\sum\left(k_{i}+p\right) \delta_{i}+\sum l_{j} \epsilon_{j}\right) \\
& =\operatorname{Stab}_{W}\left(\sum k_{i} \delta_{i}+\sum l_{j} \epsilon_{j}\right)=\operatorname{Stab}_{W}(\lambda+\rho)
\end{aligned}
$$

since $p ; k_{1}, \ldots, k_{n}>0$. Using Lemma 8.3.4 (ii), one concludes that the $\mathfrak{g}_{0}$-central character of $M(\lambda)$ is a perfect mate for $\widetilde{\chi}$.

8.7. The case when $\tilde{\chi}$ is not generic. Suppose that $\mathfrak{g}$ is of the type $D(m, n)$, $D(1,2, \alpha)$ or $F(4)$ and $\tilde{\chi}$ is not generic. 
8.7.1. Case $D(m, n)$. Since $\tilde{\chi}$ is not generic, for any $\lambda \in W(\widetilde{\chi})$ there exists $i \in$ $\{1, \ldots, n\}$ such that $(\lambda+\rho)_{\delta_{i}}=0$. On the other hand, $T^{2} \notin \tilde{\chi}$ implies $(\lambda+\rho, \beta) \neq 0$ for any $\beta \in \Delta_{1}$. In particular, $\left(\lambda+\rho, \delta_{i}+\epsilon_{j}\right) \neq 0$ and so $(\lambda+\rho)_{\epsilon_{j}} \neq 0$ for $j=1, \ldots, m$. Taking into account that $W_{1}$ acts on $\left\{\delta_{i}\right\}_{1}^{n}$ by signed permutations and $W_{2}$ acts on $\left\{\epsilon_{j}\right\}_{1}^{m}$ by signed permutations changing an even number of signs, one concludes the existence of $\lambda \in W(\widetilde{\chi})$ such that

$$
\lambda+\rho=\sum_{i=1}^{n} k_{i} \delta_{i}+\sum_{j=1}^{m} l_{j} \epsilon_{j}
$$

where

$$
\begin{aligned}
& k_{1} \geq k_{2} \geq \cdots \geq k_{n-d}>k_{n-d+1}=k_{n-d+2}=\cdots=k_{n}=0, \\
& l_{1} \geq l_{2} \geq \cdots \geq l_{m-1}>0, l_{m-1} \geq l_{m}, l_{m-1} \geq-l_{m}, l_{m} \neq 0 .
\end{aligned}
$$

Fix $\lambda$ as above and set

$$
\gamma_{d}:=\left\{\delta_{i}-\epsilon_{j}: n-d<i \leq n, 1 \leq j \leq m-1\right\} \cup\left\{\delta_{i}-\operatorname{sn}\left(l_{m}\right) \epsilon_{m}: n-d<i \leq n\right\}
$$

where $\operatorname{sn}\left(l_{m}\right):=1$ if $l_{m}>0$ and $\operatorname{sn}\left(l_{m}\right):=-1$ if $l_{m}<0$.

Let us show that the $\mathfrak{g}_{0}$-central character of $M\left(\lambda-\left|\gamma_{d}\right|\right)$ is a perfect mate for $\tilde{\chi}$. To verify that it is a mate suppose that $\left(\lambda-\gamma_{d}+\rho_{0}\right)=w\left(\lambda-\gamma+\rho_{0}\right)$ for some $\gamma \in \Gamma, w \in W$. Write

$$
|\gamma|=\sum_{1}^{n} s_{i} \delta_{i}+\sum_{1}^{m} r_{j} \epsilon_{j}
$$

Observe that $s_{i} \in\{0,1, \ldots, 2 m\}$ for $i=1, \ldots, n$. One has

$$
\begin{aligned}
\lambda-|\gamma|_{d}+\rho_{0} & =\lambda+\rho+\left(\rho_{1}-\left|\gamma_{d}\right|\right) \\
& =\sum_{1}^{n-d}\left(k_{i}+m\right) \delta_{i}+\sum_{1}^{m-1}\left(l_{j}+d\right) \epsilon_{j}+\left(l_{m}+\operatorname{sn}\left(l_{m}\right) d\right) \epsilon_{m}
\end{aligned}
$$

and

$$
\lambda+\rho_{0}-|\gamma|=\sum_{1}^{n-d}\left(k_{i}+m-s_{i}\right) \delta_{i}+\sum_{n-d+1}^{n}\left(m-s_{i}\right) \delta_{i}+\sum_{1}^{m}\left(l_{j}-r_{j}\right) \epsilon_{j} .
$$

Write $w=w_{1} w_{2}$ where $w_{1} \in W_{1}, w_{2} \in W_{2}$. Then

$$
\sum_{1}^{n-d}\left(k_{i}+m\right) \delta_{i}=w_{1}\left(\sum_{1}^{n-d}\left(k_{i}+m-s_{i}\right) \delta_{i}+\sum_{n-d+1}^{n}\left(m-s_{i}\right) \delta_{i}\right) .
$$

For any indexes $i, i^{\prime}$ such that $1 \leq i \leq n-d<i^{\prime} \leq n$ one has $k_{i}+m>m \geq$ $\pm\left(m-s_{i^{\prime}}\right)$. Therefore $w_{1}=w_{1}^{\prime} w_{1}^{\prime \prime}$ where $w_{1}^{\prime}\left(\right.$ resp., $\left.w_{1}^{\prime \prime}\right)$ is a signed permutation of $\left\{\delta_{i}\right\}_{1}^{n-d}$ (resp., of $\left.\left\{\delta_{i}\right\}_{n-d+1}^{n}\right)$. Then $\sum_{1}^{n-d}\left(k_{i}+m\right) \delta_{i}=w_{1}^{\prime}\left(\sum_{1}^{n-d}\left(k_{i}+m-s_{i}\right) \delta_{i}\right)$ and so $s_{1}=\cdots=s_{n-d}=0$ by Lemma 8.5.2 On the other hand,

$$
0=w_{1}^{\prime \prime}\left(\sum_{n-d+1}^{n}\left(m-s_{i}\right) \delta_{i}\right)
$$

gives $s_{n-d}=\cdots=s_{n}=m$.

It is easy to see that $s_{1}=\cdots=s_{n-d}=0$ implies $r_{j} \in\{0, \pm 1, \ldots, \pm d\}$ for all $j=1, \ldots, m$. 
Suppose that $l_{m}>0$. Then

$$
w_{2}\left(\sum_{1}^{m}\left(l_{j}-r_{j}\right) \epsilon_{j}\right)=\sum_{1}^{m}\left(l_{j}+d\right) \epsilon_{j}
$$

and, by Lemma 8.5.2, $r_{j}=-d$ for all $j=1, \ldots, m$. Thus $|\gamma|=\left|\gamma_{d}\right|$. It is easy to check that this implies the required equality $\gamma=\gamma_{d}$.

Suppose that $l_{m}<0$. Then

$$
w_{2}\left(\sum_{1}^{m}\left(l_{j}-r_{j}\right) \epsilon_{j}\right)=\sum_{1}^{m-1}\left(l_{j}+d\right) \epsilon_{j}+\left(l_{m}-d\right) \epsilon_{m}
$$

where $w_{2}$ is a signed permutation of $\left\{\epsilon_{j}\right\}_{1}^{m}$. Then

$$
w_{2}^{\prime}\left(\sum_{1}^{m-1}\left(l_{j}-r_{j}\right) \epsilon_{j}+\left(r_{m}-l_{m}\right) \epsilon_{m}\right)=\sum_{1}^{m-1}\left(l_{j}+d\right) \epsilon_{j}+\left(-l_{m}+d\right) \epsilon_{m}
$$

for another signed permutation $w_{2}^{\prime}$ of $\left\{\epsilon_{j}\right\}_{1}^{m}$. Since $l_{1}, \ldots, l_{m-1},-l_{m}>0$, Lemma 8.5.2 gives $r_{1}=\cdots=r_{m-1}=d$ and $r_{m}=-d$. Thus again $|\gamma|=\left|\gamma_{d}\right|$ and so $\gamma=\gamma_{d}$. Hence the $\mathfrak{g}_{0}$-central character of $M\left(\lambda-|\gamma|{ }_{d}\right)$ is a mate for $\widetilde{\chi}$.

One has

$$
\begin{aligned}
\operatorname{Stab}_{W}(\lambda+\rho) & =\operatorname{Stab}_{W}\left(\sum_{1}^{n-d} k_{i} \delta_{i}+\sum_{1}^{m} l_{j} \epsilon_{j}\right) \\
& =\operatorname{Stab}_{W}\left(\sum_{1}^{n-d}\left(k_{i}+m\right) \delta_{i}+\sum_{1}^{m-1}\left(l_{j}+d\right) \epsilon_{j}+\left(l_{m}+\operatorname{sn}\left(l_{m}\right) d\right) \epsilon_{m}\right) \\
& =\operatorname{Stab}_{W}\left(\lambda-\left|\gamma_{d}\right|+\rho_{0}\right)
\end{aligned}
$$

since $m ; d ; k_{1}, \ldots, k_{n} ; l_{1}, \ldots, l_{m-1}>\operatorname{sn}\left(l_{m}\right) l_{m}>0$. Using Lemma 8.3 .4 (ii), one concludes that the $\mathfrak{g}_{0}$-central character of $M\left(\lambda-|\gamma|_{d}\right)$ is a perfect mate for $\widetilde{\chi}$.

8.7.2. Case $D(2,1, \alpha)$. Set $\delta:=\delta_{1}$. Since $\tilde{\chi}$ is not generic, $(\lambda+\rho)_{\delta}=0$ for any $\lambda \in W(\widetilde{\chi})$. On the other hand, $T^{2} \notin \tilde{\chi}$ implies $(\lambda+\rho, \beta) \neq 0$ for any $\beta \in \Delta_{1}$. Since $\Delta_{1}^{+}=\left\{\delta \pm \epsilon_{1} \pm \epsilon_{2}\right\}$, one obtains $(\lambda+\rho)_{\epsilon_{j}} \neq 0$ for $j=1$ or $j=2$. Recall that $W \cong \mathbb{Z}_{2} \times \mathbb{Z}_{2} \times \mathbb{Z}_{2}$ where the copies of $\mathbb{Z}_{2}$ act by sign on $\delta ; \epsilon_{1} ; \epsilon_{2}$, respectively. Therefore there exists $\lambda \in W(\widetilde{\chi})$ such that

$$
\lambda+\rho=l_{1} \epsilon_{1}+l_{2} \epsilon_{2}
$$

where $l_{1}, l_{2} \geq 0$ and at least one of $l_{1}, l_{2}$ is non-zero. Fix such a $\lambda$. Both cases $l_{1}>0$ and $l_{2}>0$ are similar so we can assume $l_{1} \neq 0$. Set

$$
\gamma_{d}:=\left\{\delta-\epsilon_{1}-\epsilon_{2} ; \delta-\epsilon_{1}+\epsilon_{2}\right\} .
$$

Let us show that the $\mathfrak{g}_{0}$-central character of $M\left(\lambda-\left|\gamma_{d}\right|\right)$ is a perfect mate for $\widetilde{\chi}$.

One has $\rho_{1}-\left|\gamma_{d}\right|=2 \epsilon_{1}$ and

$$
\lambda-\left|\gamma_{d}\right|+\rho_{0}=\lambda+\rho+\rho_{1}-\left|\gamma_{d}\right|=\left(l_{1}+2\right) \epsilon_{1}+l_{2} \epsilon_{2} .
$$

Assume that $\left(\lambda-\left|\gamma_{d}\right|+\rho_{0}\right) \in W\left(\lambda-|\gamma|+\rho_{0}\right)$ for some $\gamma \in \Gamma$. Then $\left(\lambda-|\gamma|+\rho_{0}\right)_{\epsilon_{1}}=$ $\pm\left(l_{1}+2\right)$, that is, $|\gamma|_{\epsilon_{1}}=l_{1} \pm\left(l_{1}+2\right)$. Taking into account that $l_{1}>0$ and that 
$|\gamma|_{\epsilon_{1}} \in\{0, \pm 1, \pm 2\}$, one concludes $|\gamma|_{\epsilon_{1}}=-2$. This implies $\gamma=\gamma_{d}$. Hence the $\mathfrak{g}_{0}$-central character of $M\left(\lambda-\left|\gamma_{d}\right|\right)$ is a mate for $\widetilde{\chi}$. Since $l_{1}>0$

$$
\begin{aligned}
\operatorname{Stab}_{W}(\lambda+\rho) & =\operatorname{Stab}_{W}\left(l_{1} \epsilon_{1}+l_{2} \epsilon_{2}\right)=\operatorname{Stab}_{W}\left(\left(l_{1}+2\right) \epsilon_{1}+l_{2} \epsilon_{2}\right) \\
& =\operatorname{Stab}_{W}\left(\lambda-\left|\gamma_{d}\right|+\rho_{0}\right) .
\end{aligned}
$$

By Lemma 8.3.4, the $\mathfrak{g}_{0}$-central character of $M\left(\lambda-\left|\gamma_{d}\right|\right)$ is a perfect mate for $\widetilde{\chi}$.

8.7.3. Case $F(4)$. Set $\delta:=\delta_{1}$. Since $\tilde{\chi}$ is not generic, $(\lambda+\rho)_{\delta}=0$ for any $\lambda \in$ $W(\tilde{\chi})$. On the other hand, $T^{2} \notin \tilde{\chi}$ implies $(\lambda+\rho, \beta) \neq 0$ for any $\beta \in \Delta_{1}$. Since $\Delta_{1}^{+}=\left\{\frac{1}{2}\left(\delta \pm \epsilon_{1} \pm \epsilon_{2} \pm \epsilon_{3}\right)\right\}$, one obtains $(\lambda+\rho)_{\epsilon_{j}} \neq 0$ for some $j \in\{1,2,3\}$. Recall that $W=W_{1} \times W_{2}$ where $W_{1} \cong \mathbb{Z}_{2}$ acts by sign on $\delta$ and $W_{2}$ is the group of signed permutations of $\left\{\epsilon_{j}\right\}_{1}^{3}$. Therefore there exists $\lambda \in W(\widetilde{\chi})$ such that

$$
\lambda+\rho=l_{1} \epsilon_{1}+l_{2} \epsilon_{2}+l_{3} \epsilon_{3}
$$

where

$$
l_{1} \geq l_{2} \geq l_{3} \geq 0 \quad \& \quad l_{1} \neq 0
$$

Fix such a $\lambda$. Set

$$
\begin{aligned}
& \gamma_{d}:=\left\{\frac{1}{2}\left(\delta-\epsilon_{1} \pm \epsilon_{2} \pm \epsilon_{3}\right)\right\} \quad \text { if } l_{1}>l_{2}, \\
& \gamma_{d}:=\left\{\frac{1}{2}\left(\delta-\epsilon_{1}-\epsilon_{2}-\epsilon_{3}\right) ;\right. \frac{1}{2}\left(\delta+\epsilon_{1}-\epsilon_{2}-\epsilon_{3}\right) ; \\
&\left.\frac{1}{2}\left(\delta-\epsilon_{1}+\epsilon_{2}-\epsilon_{3}\right) ; \frac{1}{2}\left(\delta-\epsilon_{1}-\epsilon_{2}+\epsilon_{3}\right)\right\} \quad \text { if } l_{1}=l_{2} .
\end{aligned}
$$

We show below that the $\mathfrak{g}_{0}$-central character of $M\left(\lambda-\left|\gamma_{d}\right|\right)$ is a perfect mate for $\tilde{\chi}$.

Indeed, assume that $\left(\lambda-\left|\gamma_{d}\right|+\rho_{0}\right)=w\left(\lambda-|\gamma|+\rho_{0}\right)$ for some $\gamma \in \Gamma, w \in W$. Write $|\gamma|=s \delta+\sum_{1}^{3} r_{j} \epsilon_{j}$ and $w=w_{1} w_{2}$ where $w_{1} \in W_{1}, w_{2} \in W_{2}$. One has

$$
\lambda-|\gamma|+\rho_{0}=(2-s) \delta+\sum_{1}^{3}\left(l_{j}-r_{j}\right) \epsilon_{j} .
$$

Suppose $l_{1}>l_{2}$. Then

$$
\left|\gamma_{d}\right|=2 \delta-2 \epsilon_{1}, \quad \text { that is, } \quad \lambda-\left|\gamma_{d}\right|+\rho_{0}=\left(l_{1}+2\right) \epsilon_{1}+l_{2} \epsilon_{2}+l_{3} \epsilon_{3} .
$$

The equality $\left(\lambda-\left|\gamma_{d}\right|+\rho_{0}\right)=w\left(\lambda-|\gamma|+\rho_{0}\right)$ implies $s=2$. Thus $\gamma$ contains 4 elements and so $r_{1}, r_{2}, r_{3} \in\{0 ; \pm 1 ; \pm 2\}$. Then $l_{1}+2>l_{j}-r_{j}$ for $j=1,2$ and $l_{1}+2>-\left(l_{j}-r_{j}\right)$ for $j=1,2,3$. Thus $w_{2}\left(\epsilon_{1}\right)=\epsilon_{1}$ and so $r_{1}=-2$. Since $\gamma$ contains 4 elements, this implies $\gamma=\gamma_{d}$. Hence the $\mathfrak{g}_{0}$-central character of $M\left(\lambda-\left|\gamma_{d}\right|\right)$ is a mate for $\tilde{\chi}$.

Suppose $l_{1}=l_{2}$. Then

$$
\left|\gamma_{d}\right|=2 \delta-\epsilon_{1}-\epsilon_{2}-\epsilon_{3}, \quad \text { that is, } \quad \lambda-\left|\gamma_{d}\right|+\rho_{0}=\sum_{1}^{3}\left(l_{j}+1\right) \epsilon_{j} .
$$

Again $\gamma$ contains 4 elements and so $r_{1}, r_{2}, r_{3} \in\{0 ; \pm 1 ; \pm 2\}$. Assume that $w_{2}$ is not a usual permutation, that is, $w_{2}\left(\epsilon_{j}\right)=-\epsilon_{j^{\prime}}$ for some $j, j^{\prime}$. Then $l_{j^{\prime}}+1=$ $-\left(l_{j}-r_{j}\right)$, that is, $r_{j}-1=l_{j^{\prime}}+l_{j}>0$ because $l_{1}=l_{2}>0$ and $l_{3} \geq 0$. Then $r_{j}=2$ and, consequently, $r_{j^{\prime \prime}}=0$ for all $j^{\prime \prime} \neq j$. In particular, $w_{2}$ can change a sign of at most one of $\epsilon_{1}, \epsilon_{2}, \epsilon_{3}$. Therefore $l_{1}+1=l_{2}+1=l_{j^{\prime \prime}}-r_{j^{\prime \prime}}$ for some $j^{\prime \prime} \neq j$. Then 
$l_{1}+1=l_{j^{\prime}}$ which contradicts the inequality $l_{1} \geq l_{3}$. Thus $w_{2}$ is a usual permutation. This implies $\sum_{1}^{3}\left(l_{j}+1\right)=\sum_{1}^{3}\left(l_{j}-r_{j}\right)$, that is, $r_{1}+r_{2}+r_{3}=-3$. Taking into account that $r_{j} \in\{0 ; \pm 1 ; \pm 2\}$ and that the equality of the form $r_{j}= \pm 2$ implies $r_{j^{\prime}}=0$ for $j^{\prime} \neq j$, one concludes $r_{1}=r_{2}=r_{3}=-1$, that is, $|\gamma|=\left|\gamma_{d}\right|$. It is easy to check that this gives the required equality $\gamma=\gamma_{d}$. Hence the $\mathfrak{g}_{0}$-central character of $M\left(\lambda-\left|\gamma_{d}\right|\right)$ is a mate for $\tilde{\chi}$.

If $l_{1}>l_{2} \geq l_{3} \geq 0$, then

$$
\begin{aligned}
\operatorname{Stab}_{W}(\lambda+\rho) & =\operatorname{Stab}_{W}\left(l_{1} \epsilon_{1}+l_{2} \epsilon_{2}+l_{3} \epsilon_{3}\right) \\
& =\operatorname{Stab}_{W}\left(\left(l_{1}+2\right) \epsilon_{1}+l_{2} \epsilon_{2}+l_{3} \epsilon_{3}\right) \\
& =\operatorname{Stab}_{W}\left(\lambda-\left|\gamma_{d}\right|+\rho_{0}\right) .
\end{aligned}
$$

If $l_{1}=l_{2}>0$, then $l_{3} \neq 0$ since $\left(\lambda+\rho, \delta+\epsilon_{1}-\epsilon_{2}+\epsilon_{3}\right) \neq 0$. Therefore

$$
\begin{aligned}
\operatorname{Stab}_{W}(\lambda+\rho) & =\operatorname{Stab}_{W}\left(l_{1} \epsilon_{1}+l_{2} \epsilon_{2}+l_{3} \epsilon_{3}\right) \\
& =\operatorname{Stab}_{W}\left(\left(l_{1}+1\right) \epsilon_{1}+\left(l_{2}+1\right) \epsilon_{2}+\left(l_{3}+1\right) \epsilon_{3}\right) \\
& =\operatorname{Stab}_{W}\left(\lambda-\left|\gamma_{d}\right|+\rho_{0}\right) .
\end{aligned}
$$

Hence the $\mathfrak{g}_{0}$-central character of $M\left(\lambda-\left|\gamma_{d}\right|\right)$ is a perfect mate for $\tilde{\chi}$.

\section{Annihilation Theorem}

This section is devoted to the proof of Theorem 9.5.

9.1. Lemma. Let $\widetilde{M}$ be a strongly typical Verma module, and let $v \in \widetilde{M}$ be a primitive vector. Then $\widetilde{\mathcal{U}} v$ is a Verma module. In particular, $\widetilde{M}$ contains a simple Verma submodule.

Proof. Set $\tilde{\chi}:=\operatorname{Ann}_{\mathcal{Z}(\mathfrak{g})} \widetilde{M}$. Since $\widetilde{M}$ is strongly typical, $\widetilde{\chi}$ is also strongly typical and so for $\tilde{\chi}$ there exists a perfect mate $\chi \in \operatorname{Max} \mathcal{Z}\left(\mathfrak{g}_{0}\right)$ - see Section 8 Let $\widetilde{N}$ be a submodule of $\widetilde{M}$ generated by a primitive vector. Then $\widetilde{N}$ is a quotient of a Verma module $\widetilde{M^{\prime}}$ and $\widetilde{\chi} \widetilde{M}^{\prime}=0$. By 8.2.4 $M^{\prime}:=\widetilde{M}_{\chi}^{\prime}$ is a Verma $\mathfrak{g}_{0}$-module. Since $\chi$ is a perfect mate for $\tilde{\chi}$, the $\mathfrak{g}_{0}$-module $\widetilde{N}_{\chi}$ is a non-zero quotient of $M^{\prime}$. Taking into account that $\widetilde{N}_{\chi} \subset \widetilde{M}$ is torsion-free over $\mathcal{U}\left(\mathfrak{n}_{0}^{-}\right)$, one concludes $\widetilde{N}_{\chi}=M^{\prime}$. Thus $\left(\widetilde{M}^{\prime} / \widetilde{N}\right)_{\chi}=0$ and so $\widetilde{N}=\widetilde{M}^{\prime}$ is a Verma module.

Recall that $\widetilde{M}$ has a finite length and so it contains a simple submodule $\widetilde{N}$. A highest weight vector of $\widetilde{N}$ is primitive. Hence $\widetilde{N}$ is a simple Verma module.

9.2. Lemma. Let $\widetilde{M}$ be a strongly typical Verma module, and let $\widetilde{M^{\prime}}$ be a simple submodule of $\widetilde{M}$. Then the natural maps $F(\widetilde{M}, \widetilde{M}) \rightarrow F\left(\widetilde{M^{\prime}}, \widetilde{M}\right)$ and $F\left(\widetilde{M}^{\prime}, \widetilde{M}^{\prime}\right) \rightarrow$ $F\left(\widetilde{M^{\prime}}, \widetilde{M}\right)$ are $\mathfrak{g}$-bimodule isomorphisms.

Proof. Denote by $\iota$ the natural map $F(\widetilde{M}, \widetilde{M}) \rightarrow F\left(\widetilde{M^{\prime}}, \widetilde{M}\right)$ and by $\iota^{\prime}$ the natural map $F\left(\widetilde{M^{\prime}}, \widetilde{M}^{\prime}\right) \rightarrow F\left(\widetilde{M^{\prime}}, \widetilde{M}\right)$ (both maps are induced by the embedding $\widetilde{M}^{\prime}$ to $\widetilde{M})$. Both maps are $\mathfrak{g}$-bimodule homomorphisms.

To show that $\iota^{\prime}$ is a bijection and that $\iota$ is a monomorphism, we use the following reasoning which is essentially the same as in [J1, Section 6, and [J2], 8.3.9.

By [J1], $F\left(N_{1}, N_{2}\right)=0$ if the GK-dimension of any simple quotient of $N_{1}$ is greater than the GK-dimension of $N_{2}$ or if the GK-dimension of $N_{1}$ is less than the GK-dimension of any simple submodule of $N_{2}$. Moreover, the GK-dimension of a Verma module is equal to the GK-dimension of the algebra $\mathcal{U}\left(\mathfrak{n}^{-}\right)$. 
Let $v$ be a highest weight vector of $\widetilde{M}$, and let $u \in \mathcal{U}\left(\mathfrak{n}^{-}\right)$be such that $u v$ is a highest weight vector of $\widetilde{M^{\prime}}$. Since $\widetilde{M^{\prime}}$ is a Verma module, $u$ is a non-zero divisor in $\mathcal{U}\left(\mathfrak{n}^{-}\right)$. This implies

$$
G K \operatorname{dim} \widetilde{M^{\prime}}=G K \operatorname{dim} \widetilde{M}>G K \operatorname{dim} \widetilde{M} / \widetilde{M^{\prime}} .
$$

The only simple quotient of $\widetilde{M^{\prime}}$ is $\widetilde{M^{\prime}}$ itself; thus the inequality $G K \operatorname{dim} \widetilde{M^{\prime}}>$ $G K \operatorname{dim} \widetilde{M} / \widetilde{M^{\prime}}$ implies $F\left(\widetilde{M^{\prime}}, \widetilde{M} / \widetilde{M}^{\prime}\right)=0$. Therefore $\iota^{\prime}$ is an epimorphism. Obviously, $\iota^{\prime}$ is a monomorphism. Hence $\iota^{\prime}$ is bijective. By Lemma 9.1, any simple submodule of $\widetilde{M}$ is a Verma module. Thus the GK-dimension of $\widetilde{M} / \widetilde{M^{\prime}}$ is less than the GK-dimension of any simple submodule of $\widetilde{M}$ and so $F\left(\widetilde{M} / \widetilde{M^{\prime}}, \widetilde{M}\right)=0$. Consequently, $\iota$ is a monomorphism.

It remains to verify the surjectivity of $\iota$. Since $\iota^{\prime}$ is bijective, it is enough to check the surjectivity of the composed map $\iota^{\prime \prime}:=\left(\iota^{\prime}\right)^{-1} \circ \iota: F(\widetilde{M}, \widetilde{M}) \rightarrow F\left(\widetilde{M}^{\prime}, \widetilde{M^{\prime}}\right)$. Denote by $N$ the cokernel of the map $\iota^{\prime \prime}$. This is a $\mathfrak{g}$-bimodule and its left and right annihilators contain $\tilde{\chi}:=\operatorname{Ann}_{\mathcal{Z}(\mathfrak{g})} \widetilde{M}$. Let $\chi \in \operatorname{Max} \mathcal{Z}\left(\mathfrak{g}_{0}\right)$ be a perfect mate for $\tilde{\chi}$. Using the notation of 8.4 .2 , one has

$$
{ }_{\chi} F(\widetilde{M}, \widetilde{M})_{\chi} \stackrel{\sim}{\longrightarrow} F\left(\widetilde{M}_{\chi}, \widetilde{M}_{\chi}\right), \quad{ }_{\chi} F\left(\widetilde{M}^{\prime}, \widetilde{M}^{\prime}\right)_{\chi} \stackrel{\sim}{\longrightarrow} F\left(\widetilde{M}_{\chi}^{\prime}, \widetilde{M}_{\chi}^{\prime}\right)
$$

Since $\chi$ is a mate for $\widetilde{\chi}$, both $M:=\widetilde{M}_{\chi}$ and $M^{\prime}:=\widetilde{M}_{\chi}^{\prime}$ are Verma $\mathfrak{g}_{0}$-modules. For any $V \in \operatorname{Irr}_{0}$ one has $\operatorname{Hom}_{\mathfrak{g}_{0}}(V, F(M, M))=\left.\operatorname{dim} V\right|_{0}=\operatorname{Hom}_{\mathfrak{g}_{0}}\left(V, F\left(M^{\prime}, M^{\prime}\right)\right)$ (see JJ1, 6.4). Taking into account the injectivity of $\iota^{\prime \prime}$, one concludes that $\iota^{\prime \prime}(F(M, M))$ $=F\left(M^{\prime}, M^{\prime}\right)$ and so ${ }_{\chi} N_{\chi}=0$. Thus $N=0$ by 8.4.2. Hence $\iota, \iota^{\prime}$ are isomorphisms. The lemma is proved.

Using Lemma 9.1 one obtains the

9.2.1. Corollary. Let $\widetilde{M}, \widetilde{M^{\prime}}$ be strongly typical Verma modules, and let $\widetilde{M^{\prime}}$ be a submodule of $\widetilde{M}$. Then the $\mathfrak{g}$-bimodules $F(\widetilde{M}, \widetilde{M})$ and $F\left(\widetilde{M^{\prime}}, \widetilde{M}^{\prime}\right)$ are isomorphic.

9.3. Proposition. If $\widetilde{M}$ is a strongly typical projective (in $\widetilde{\mathcal{O}})$ Verma module, then

$$
F(\widetilde{M}, \widetilde{M}) \cong \underset{\widetilde{V} \in \operatorname{Irr}}{ } \mathrm{E}(\widetilde{V})^{\left.\oplus \operatorname{dim} \tilde{V}\right|_{0}}
$$

Proof. Step 1. Let us show that for any $\mathfrak{g}_{0}$-modules $N_{1}, N_{2}$ the following ad $\mathfrak{g}$ modules are isomorphic:

$$
L:=F\left(\operatorname{Ind}_{\mathfrak{g}_{0}}^{\mathfrak{g}} N_{1}, \operatorname{Ind}_{\mathfrak{g}_{0}}^{\mathfrak{g}} N_{2}\right), \quad L^{\prime}:=\operatorname{Coind}_{\mathfrak{g}_{0}}^{\mathfrak{g}}\left(F\left(N_{1}, N_{2}\right) \otimes \Lambda \mathfrak{g}_{1}\right) .
$$

Indeed, using the Frobenius reciprocity, one obtains

$$
\begin{aligned}
\operatorname{Hom}_{\mathfrak{g}_{0}}\left(V, F\left(\operatorname{Ind}_{\mathfrak{g}_{0}}^{\mathfrak{g}} N_{1}, \operatorname{Ind}_{\mathfrak{g}_{0}}^{\mathfrak{g}} N_{2}\right)\right) & \cong \operatorname{Hom}_{\mathfrak{g}_{0}}\left(V, F\left(N_{1}, N_{2}\right) \otimes \Lambda \mathfrak{g}_{1} \otimes \Lambda \mathfrak{g}_{1}\right) \\
& \cong \operatorname{Hom}_{\mathfrak{g}_{0}}\left(V, \operatorname{Coind}_{\mathfrak{g}_{0}}^{\mathfrak{g}}\left(F\left(N_{1}, N_{2}\right) \otimes \Lambda \mathfrak{g}_{1}\right)\right)
\end{aligned}
$$

for any $V \in \operatorname{Irr}_{0}$. Hence $L \cong L^{\prime}$ as ad $\mathfrak{g}_{0}$-modules. Note that $\operatorname{dim} \operatorname{Hom}_{\mathfrak{g}_{0}}(V, L)<\infty$ for all $V \in \operatorname{Irr}_{0}$. 
On the other hand, for any $\widetilde{V} \in \operatorname{Irr}$ one has

$$
\begin{aligned}
\operatorname{Hom}_{\mathfrak{g}}\left(\widetilde{V}, F\left(\operatorname{Ind}_{\mathfrak{g}_{0}}^{\mathfrak{g}} N_{1}, \operatorname{Ind}_{\mathfrak{g}_{0}}^{\mathfrak{g}} N_{2}\right)\right) & \cong \operatorname{Hom}_{\mathfrak{g}}\left(\operatorname{Ind}_{\mathfrak{g}_{0}}^{\mathfrak{g}} N_{1}, \operatorname{Ind}_{\mathfrak{g}_{0}}^{\mathfrak{g}} N_{2} \otimes \widetilde{V}^{*}\right) \\
& \cong \operatorname{Hom}_{\mathfrak{g}_{0}}\left(N_{1}, \operatorname{Ind}_{\mathfrak{g}_{0}}^{\mathfrak{g}} N_{2} \otimes \widetilde{V}^{*}\right) \\
& \cong \operatorname{Hom}_{\mathfrak{g}_{0}}\left(N_{1}, N_{2} \otimes \Lambda \mathfrak{g}_{1} \otimes \widetilde{V}^{*}\right) \\
& \cong \operatorname{Hom}_{\mathfrak{g}_{0}}\left(\widetilde{V}, F\left(N_{1}, N_{2}\right) \otimes \Lambda \mathfrak{g}_{1}\right) \\
& \cong \operatorname{Hom}_{\mathfrak{g}}\left(\widetilde{V}, \operatorname{Coind}_{\mathfrak{g}_{0}}^{\mathfrak{g}}\left(F\left(N_{1}, N_{2}\right) \otimes \Lambda \mathfrak{g}_{1}\right)\right)
\end{aligned}
$$

Hence $\operatorname{Soc} L \cong \operatorname{Soc} L^{\prime}$. Since $L^{\prime}$ is injective in $\mathcal{F}$ in, it contains a submodule isomorphic to $L$. Taking into account the ad $\mathfrak{g}_{0}$-isomorphism $L \cong L^{\prime}$, one concludes that $L \cong L^{\prime}$ as ad $\mathfrak{g}$-modules.

Step 2. Let $\chi$ be a perfect mate of $\operatorname{Ann}_{\mathcal{Z}(\mathfrak{g})} \widetilde{M}$. Then $M:=\widetilde{M}_{\chi}$ is a Verma $\mathfrak{g}_{0}$-module and $\widetilde{M}=\widetilde{\mathcal{U}} M$ by Corollary 8.3.2. Thus $\widetilde{M}$ is a quotient of $\operatorname{Ind}_{\mathfrak{g}_{0}}^{\mathfrak{g}} M$. Since $\widetilde{M}$ is projective, it is a direct summand of $\operatorname{Ind}_{\mathfrak{g}_{0}}^{\mathfrak{g}} M$. Then the ad $\mathfrak{g}$-module $F(\widetilde{M}, \widetilde{M})$ is a direct summand of the ad $\mathfrak{g}$-module $F\left(\operatorname{Ind}_{\mathfrak{g}_{0}}^{\mathfrak{g}} M, \operatorname{Ind}_{\mathfrak{g}_{0}}^{\mathfrak{g}} M\right)$. The last is isomorphic to $\operatorname{Coind}_{\mathfrak{g}_{0}}^{\mathfrak{g}}\left(F(M, M) \otimes \Lambda \mathfrak{g}_{1}\right)$ and so is injective in $\mathcal{F}$ in. Hence the ad $\mathfrak{g}$-module $F(\widetilde{M}, \widetilde{M})$ is injective in $\mathcal{F}$ in.

By Lemma 9.1, $\widetilde{M}$ contains a simple Verma submodule $\widetilde{M}^{\prime}$. Combining Corollary 9.2 .1 and 11.4 .1 one obtains

$$
\operatorname{Soc} F(\widetilde{M}, \widetilde{M}) \cong \operatorname{Soc} F\left(\widetilde{M}^{\prime}, \widetilde{M}^{\prime}\right) \cong \bigoplus_{\widetilde{V} \in \operatorname{Irr}} \widetilde{V}^{\left.\oplus \operatorname{dim} \tilde{V}\right|_{0}}
$$

Now the injectivity of $F(\widetilde{M}, \widetilde{M})$ implies the required assertion.

9.4. Proposition. If $\widetilde{M}$ is a strongly typical Verma module, then the natural map $\widetilde{\mathcal{U}} \rightarrow F(\widetilde{M}, \widetilde{M})$ is surjective.

Proof. Denote by $N$ the cokernel of the natural map $f: \widetilde{\mathcal{U}} \rightarrow F(\widetilde{M}, \widetilde{M})$. This is a $\mathfrak{g}$-bimodule and its left and right annihilators contain $\widetilde{\chi}:=\operatorname{Ann}_{\mathcal{Z}(\mathfrak{g})} \widetilde{M}$. Let $\chi \in \operatorname{Max} \mathcal{Z}\left(\mathfrak{g}_{0}\right)$ be a perfect mate for $\tilde{\chi}$. Using the notation of 8.4 .2 one has ${ }_{\chi} F(\widetilde{M}, \widetilde{M})_{\chi} \stackrel{\sim}{\longrightarrow} F\left(\widetilde{M}_{\chi}, \widetilde{M}_{\chi}\right)$. Since $\chi$ is a mate for $\widetilde{\chi}, M:=\widetilde{M}_{\chi}$ is a Verma $\mathfrak{g}_{0}$-module. As a $\mathfrak{g}_{0}$-module, $\widetilde{M}$ has a finite length and so

$$
\operatorname{Ann}_{\mathcal{Z}\left(\mathfrak{g}_{0}\right)} \widetilde{M}=\chi \prod_{i=1}^{k} \chi_{i}^{r_{i}}
$$

where $\left\{\chi, \chi_{1}, \ldots, \chi_{k}\right\}=\operatorname{supp}_{\mathcal{Z}\left(\mathfrak{g}_{0}\right)} \widetilde{M}$ and $r_{1}, \ldots, r_{k} \in \mathbb{N}^{+}$. Any element of $\prod_{i=1}^{k} \chi_{i}^{r_{i}}$ annihilates $\sum_{i=1}^{k} \widetilde{M}_{\chi_{i}}$ and acts by scalar on $\widetilde{M}_{\chi}$. Taking into account that the natural map $\mathcal{U} \rightarrow F(M, M)$ is surjective (see [J1], 6.4), one concludes that

$$
f\left(\mathcal{U} \prod_{i=1}^{k} \chi_{i}^{r_{i}}\right)={ }_{\chi} F(\widetilde{M}, \widetilde{M})_{\chi},
$$

and thus ${ }_{\chi} N_{\chi}=0$. By 8.4.2 $N=0$ as required.

9.5. Theorem. For a strongly typical Verma $\mathfrak{g}$-module $\widetilde{M}$

$$
\operatorname{Ann} \widetilde{M}=\widetilde{\mathcal{U}} \operatorname{Ann}_{\mathcal{Z}(\mathfrak{g})} \widetilde{M} \text {. }
$$


Proof. Set $\widetilde{\chi}:=\operatorname{Ann}_{\mathcal{Z}(\mathfrak{g})} \widetilde{M}$. Let $\widetilde{M}^{\prime \prime}$ be a projective (in $\widetilde{\mathcal{O}}$ ) Verma module such that $\widetilde{\chi} \widetilde{M}^{\prime \prime}=0$ and let $\widetilde{M^{\prime}}$ be a simple submodule of $\widetilde{M^{\prime \prime}}$ (by $9.1 \widetilde{M}^{\prime}$ is a Verma module). Combining 9.2.1 and 9.3, one obtains

$$
F\left(\widetilde{M}^{\prime}, \widetilde{M}^{\prime}\right) \cong \bigoplus_{\widetilde{V} \in \operatorname{Irr}} \mathrm{E}(\widetilde{V})^{\left.\oplus \operatorname{dim} \widetilde{V}\right|_{0}}
$$

Summarizing 5.1, 5.2 and 9.4, one concludes that Ann $\widetilde{M}^{\prime}=\widetilde{\mathcal{U}} \widetilde{\chi}$ and that the natural map $f^{\prime}: \widetilde{\mathcal{U}} /(\widetilde{\mathcal{U}} \widetilde{\chi}) \rightarrow F\left(\widetilde{M}^{\prime}, \widetilde{M}^{\prime}\right)$ is bijective.

Denote by $f$ the natural map $\widetilde{\mathcal{U}} /(\widetilde{\mathcal{U}} \widetilde{\chi}) \rightarrow F(\widetilde{M}, \widetilde{M})$ and by $p$ the composition map $f \circ\left(f^{\prime}\right)^{-1}: F\left(\widetilde{M}^{\prime}, \widetilde{M}^{\prime}\right) \rightarrow F(\widetilde{M}, \widetilde{M})$. Obviously $p$ is a $\widetilde{\mathcal{U}}$-bimodule map. By 9.4 $p$ is surjective. Let us show that $p$ is bijective.

Let $\chi$ be a perfect mate for $\tilde{\chi}$; set $M:=\widetilde{M}_{\chi}, M^{\prime}:=\widetilde{M}_{\chi}^{\prime}$. Using the notation of 8.4 .2 one has

$$
{ }_{\chi} F(\widetilde{M}, \widetilde{M})_{\chi}=F(M, M), \quad{ }_{\chi} F\left(\widetilde{M}^{\prime}, \widetilde{M}^{\prime}\right)_{\chi}=F\left(M^{\prime}, M^{\prime}\right)
$$

and so ${ }_{\chi} F(\widetilde{M}, \widetilde{M})_{\chi},{ }_{\chi} F\left(\widetilde{M^{\prime}}, \widetilde{M^{\prime}}\right)_{\chi}$ are isomorphic ad $\mathfrak{g}_{0}$-modules. It is easy to see that

$$
\chi(\operatorname{Im} p)_{\chi}=p\left({ }_{\chi} F\left(\widetilde{M}^{\prime}, \widetilde{M}^{\prime}\right)_{\chi}\right) .
$$

Therefore $p\left({ }_{\chi} F\left(\widetilde{M^{\prime}}, \widetilde{M}^{\prime}\right)_{\chi}\right)={ }_{\chi} F(\widetilde{M}, \widetilde{M})_{\chi}$ since $p$ is surjective. Taking into account that the multiplicity of each simple $\mathfrak{g}_{0}$-module $V$ in $F(M, M)$ is finite, one concludes that the restriction of $p$ to ${ }_{\chi} F\left(\widetilde{M}^{\prime}, \widetilde{M}^{\prime}\right)_{\chi}$ is a monomorphism. Thus $\chi(\operatorname{ker} p)_{\chi}=0$ and $\operatorname{ker} p=0$ by 8.4.2. This means that $p$ is bijective and so $f: \widetilde{\mathcal{U}} /(\widetilde{\mathcal{U}} \widetilde{\chi}) \rightarrow$ $F(\widetilde{M}, \widetilde{M})$ is bijective as well. The assertion follows.

9.6. Corollary. For a strongly typical central character $\tilde{\chi}$

$$
\widetilde{\mathcal{U}} /(\widetilde{\mathcal{U}} \widetilde{\chi}) \cong \bigoplus_{\tilde{V} \in \operatorname{Irr}} \mathrm{E}(\widetilde{V})^{\left.\oplus \operatorname{dim} \tilde{V}\right|_{0}}
$$

\section{Remark about Verma modules}

In this section we study the $\mathfrak{g}_{0}$-structure of Verma $\mathfrak{g}$-modules.

10.1. Retain the notation of Section 8 . Take strongly typical $\tilde{\chi} \in \operatorname{Max} \mathcal{Z}(\mathfrak{g})$ and $\lambda \in W(\widetilde{\chi})$. Recall that as a $\mathfrak{g}_{0}$-module $\widetilde{M}(\lambda)$ has a finite filtration with the factors $\{M(\lambda-|\gamma|), \gamma \in \Gamma\}$. Therefore

$$
\widetilde{\mathcal{U}} \widetilde{\chi} \cap \mathcal{Z}\left(\mathfrak{g}_{0}\right)=\operatorname{Ann} \widetilde{M}(\lambda) \cap \mathcal{Z}\left(\mathfrak{g}_{0}\right)=\prod_{i} \chi_{i}^{r_{i}}
$$

where the $\chi_{i}$ are the pairwise distinct elements of the multiset

$$
\left\{\operatorname{Ann}_{\mathcal{Z}\left(\mathfrak{g}_{0}\right)} M(\lambda-|\gamma|), \gamma \in \Gamma\right\}
$$

and the $r_{i}$ are positive integers. Thus for any $\tilde{\mathcal{U}}_{\tilde{\chi}}$-module $\widetilde{N}$

$$
\widetilde{N}=\bigoplus_{i} \tilde{N}_{\chi_{i}}, \quad \chi_{i}^{r_{i}} \widetilde{N}_{\chi_{i}}=0
$$

Suppose that $\widetilde{M}(\lambda)=\bigoplus_{\gamma \in \Gamma} M(\lambda-\gamma)$ and so $\mathcal{Z}\left(\mathfrak{g}_{0}\right)$ acts semisimply on $\widetilde{M}(\lambda)$. If, in addition, $\lambda$ is strongly typical, then (9) implies that $\mathcal{Z}\left(\mathfrak{g}_{0}\right)$ acts semisimply on any $\widetilde{\mathcal{U}}_{\tilde{\chi}}$-module for $\widetilde{\chi}=\operatorname{Ann}_{\mathcal{Z}(\mathfrak{g})} \widetilde{M}(\lambda)$. 
10.2. Proposition. For $\widetilde{M}(\lambda)$ a simple Verma module the following conditions are equivalent:

$$
\begin{array}{ll}
\text { (i) } & \widetilde{M}=\bigoplus_{\gamma \in \Gamma} M(\lambda-|\gamma|) \text {. } \\
\text { (ii) } & \forall \gamma, \gamma^{\prime} \in \Gamma\left(|\gamma|-\left|\gamma^{\prime}\right|\right) \in \mathbb{Z} \Delta_{0} \backslash\{0\} \Longrightarrow\left(\lambda-|\gamma|+\rho_{0}\right) \notin W\left(\lambda-\left|\gamma^{\prime}\right|+\rho_{0}\right) . \\
\text { (iii) } M(\lambda-|\gamma|) \text { is simple for any } \gamma \in \Gamma \text {. }
\end{array}
$$

Proof. The implication (ii) $\Longrightarrow$ (i) follows from the fact that any exact sequence $0 \rightarrow M(\mu) \rightarrow N \rightarrow M(\mu) \rightarrow 0$ in $\mathcal{O}$ splits (see, for instance, 2.5.3).

Let us verify the implication (i) $\Longrightarrow$ (iii). Recall 2.5.6 Since $\widetilde{M}(\lambda)$ is simple, it is isomorphic to $\widetilde{M}(\lambda)^{\#}=\bigoplus_{\gamma \in \Gamma} M(\lambda-|\gamma|)^{\#}$. The module $\widetilde{M}(\lambda)$ is $\mathcal{U}\left(\mathfrak{n}_{0}^{-}\right)$-torsionfree; thus all $M(\lambda-|\gamma|)^{\#}$ are also $\mathcal{U}\left(\mathfrak{n}_{0}^{-}\right)$-torsion-free. This forces (iii).

The implication (iii) $\Longrightarrow$ (ii) follows from the fact that if $M(\mu), M\left(\mu^{\prime}\right)$ are simple Verma $\mathfrak{g}_{0}$-modules with the same $\mathfrak{g}_{0}$-central character and $\left(\mu-\mu^{\prime}\right) \in \mathbb{Z} \Delta_{0}$, then $\mu=\mu^{\prime}$. This fact can be deduced from [J2], A.1.14 and A.1.1 (vii).

Hence the conditions (i), (ii) and (iii) are equivalent provided that $\widetilde{M}(\lambda)$ is simple.

10.3. Corollary. If $\widetilde{M}(\lambda)$ contains a simple typical Verma submodule, then conditions (i) and (ii) are equivalent.

Proof. The implication (ii) $\Longrightarrow$ (i) follows from the same argument as in Proposition 10.2. To verify the implication (i) $\Longrightarrow$ (ii), assume that $\widetilde{M}(\lambda)=\bigoplus M_{i}$ is a direct sum of Verma $\mathfrak{g}_{0}$-modules and that

$$
\lambda-\left|\gamma^{\prime}\right|+\rho_{0}=w\left(\lambda-\left|\gamma^{\prime \prime}\right|+\rho_{0}\right)
$$

for some $w \in W$ and $\gamma^{\prime}, \gamma^{\prime \prime} \in \Gamma$ satisfying $\left(\left|\gamma^{\prime \prime}\right|-\left|\gamma^{\prime}\right|\right) \in \mathbb{Z} \Delta_{0} \backslash\{0\}$. Take $w^{\prime} \in W$ such that $\widetilde{M}\left(w^{\prime} \cdot \lambda\right)$ is a simple submodule of $\widetilde{M}(\lambda)$. In the notation of 8.2 .2 one has

$$
\begin{aligned}
w^{\prime} . \lambda-\left|w_{*}^{\prime} \gamma^{\prime}\right|+\rho_{0} & =w^{\prime}\left(\lambda-\left|\gamma^{\prime}\right|+\rho_{0}\right)=w^{\prime} w\left(\lambda-\left|\gamma^{\prime \prime}\right|+\rho_{0}\right) \\
& =w^{\prime} w\left(w^{\prime}\right)^{-1}\left(w^{\prime} . \lambda-\left|w_{*}^{\prime} \gamma^{\prime \prime}\right|+\rho_{0}\right)
\end{aligned}
$$

by (7). Furthermore $\left|w_{*}^{\prime} \gamma^{\prime}\right|-\left|w_{*}^{\prime} \gamma^{\prime \prime}\right|=w\left(\left|\gamma^{\prime \prime}\right|-\left|\gamma^{\prime}\right|\right) \in \mathbb{Z} \Delta_{0} \backslash\{0\}$. Thus condition (ii) does not hold for a simple Verma module $\widetilde{M}\left(w^{\prime} \cdot \lambda\right)$. Therefore $\widetilde{M}\left(w^{\prime} \cdot \lambda\right)$ has a $\mathfrak{g}_{0}$-submodule $N$ which is isomorphic to a non-splitting extension of $M(\mu)$ by $M\left(\mu^{\prime}\right)$ for some $\mu, \mu^{\prime} \in\{\lambda-|\gamma|: \gamma \in \Gamma\}$. Since $\widetilde{M}\left(w^{\prime} . \lambda\right)$ is a submodule of $\widetilde{M}(\lambda)$, $N$ is a $\mathfrak{g}_{0}$-submodule of $\widetilde{M}(\lambda)$. Since $N$ is indecomposable, it is isomorphic to a submodule of a Verma module $M_{i}$. However the Gelfand-Kirillov dimension of any proper quotient of a Verma $\mathfrak{g}_{0}$-module is strictly less than the Gelfand-Kirillov dimension of $M\left(\mu^{\prime}\right)$ - see [J1]. This gives the required contradiction.

Remark. Recall that a strongly typical Verma module contains a simple strongly typical Verma submodule.

10.4. Denote by $\Gamma_{0}$ (resp., $\Gamma_{1}$ ) the set of subsets of $\Delta_{1}^{+}$containing an even (resp., an odd) number of elements. Take an arbitrary $\lambda \in \mathfrak{h}^{*}$ and fix a $\mathbb{Z}_{2}$-grading on a Verma module $\widetilde{M}(\lambda)$ in such a way that a highest weight vector becomes even. As a $\mathfrak{g}_{0}$-module, $\widetilde{M}=\widetilde{M}_{0} \oplus \widetilde{M}_{1}$. Let us show that each $\widetilde{M}(\lambda)_{j}$ has a finite filtration with factors $\left\{M(\lambda-\gamma) \mid \gamma \in \Gamma_{j}\right\}(j=0,1)$. 
Set

$$
\begin{aligned}
& Q_{0}(\pi):=\left\{\sum_{\alpha \in \Delta} k_{\alpha} \alpha \mid k_{\alpha} \in \mathbb{Z}, \sum_{\alpha \in \Delta_{1}} k_{\alpha} \text { is even }\right\}, \\
& Q_{1}(\pi):=\left\{\sum_{\alpha \in \Delta} k_{\alpha} \alpha \mid k_{\alpha} \in \mathbb{Z}, \sum_{\alpha \in \Delta_{1}} k_{\alpha} \text { is odd }\right\} .
\end{aligned}
$$

Note that both $Q_{0}(\pi), Q_{1}(\pi)$ are $W$-stable. We claim that $Q_{0}(\pi) \cap Q_{1}(\pi)=\emptyset$ (for $\mathfrak{g}=\mathfrak{p s} \mathfrak{l}(n, n)$ we substitute $\mathfrak{h}$ by $\hat{\mathfrak{h}}$ - see 6.1.4). Indeed, if $\mathfrak{g}$ is of type I, then $\mathfrak{h} \cap \mathcal{Z}\left(\mathfrak{g}_{0}\right)$ contains an element $z$ such that $z(\alpha)=1$ for any $\alpha \in \Delta_{1}^{+}$. Therefore

$$
\sum_{\alpha \in \Delta} k_{\alpha} \alpha(z)=\sum_{\alpha \in \Delta_{1}^{+}}\left(k_{\alpha}-k_{-\alpha}\right)
$$

and so $\mu(z)$ is an even (resp., an odd) integer for $\mu \in Q_{0}(\pi)$ (resp., $\mu \in Q_{1}(\pi)$ ). Let $\mathfrak{g}$ be of type II. Retain the notation of 8.5. One can immediately see that for $\mathfrak{g} \neq F(4)$ the sum $\sum_{1}^{n} \mu_{\delta_{i}}$ is an even (resp., an odd) integer for $\mu \in Q_{0}(\pi)$ (resp., $\left.\mu \in Q_{1}(\pi)\right)$. For the remaining case $\mathfrak{g}=F(4), \mu_{\delta_{1}}$ is an integer if $\mu \in Q_{0}(\pi)$ and belongs to the set $\mathbb{Z}+\frac{1}{2}$ if $\mu \in Q_{1}(\pi)$. This implies our claim.

The weights of $\widetilde{M}(\lambda)_{0}$ (resp., $\left.\widetilde{M}(\lambda)_{1}\right)$ belong to the set $\left(\lambda-Q_{0}(\pi)\right.$ ) (resp., $\left.\left(\lambda-Q_{1}(\pi)\right)\right)$. The weights of $M(\lambda-|\gamma|)$ belong to $\left(\lambda-Q_{j}(\pi)\right)$ iff $\gamma \in \Gamma_{j}(j=0,1)$. This proves that $\widetilde{M}(\lambda)_{j}$ has a finite filtration with the factors $\left\{M(\lambda-\gamma) \mid \gamma \in \Gamma_{j}\right\}$ $(j=0,1)$.

Using arguments as above, it is easy to show that for $\mathfrak{g}$ of type II

$$
Q_{0}(\pi)=\mathbb{Z} \Delta_{0},
$$

and for $\mathfrak{g}$ of type I

$$
Q_{0}(\pi) \cap\left\{\mu \in \mathfrak{h}^{*} \mid \mu(z)=0\right\}=\mathbb{Z} \Delta_{0} .
$$

This implies that condition (ii) of Proposition 10.2 is equivalent to the condition

$$
\forall \gamma^{\prime}, \gamma^{\prime \prime} \in \Gamma_{j} \quad\left(\lambda-\left|\gamma^{\prime}\right|+\rho_{0}\right) \in W\left(\lambda-\left|\gamma^{\prime \prime}\right|+\rho_{0}\right) \quad \Longrightarrow \quad\left|\gamma^{\prime}\right|=\left|\gamma^{\prime \prime}\right|
$$

for $j=0,1$.

10.5. Remark. The case $\mathfrak{g}=\mathfrak{o s p}(1,2 l)$ was treated in [M1], 3.7, and [GL2], 7.2.

\section{ApPEndix}

This section contains some lemmas used in the main text.

11.1. Recall that a simple $\mathfrak{g}_{0}$-module $V(\lambda)$ is finite dimensional iff $\lambda+\rho_{0}>$ $w\left(\lambda+\rho_{0}\right)$ for any $w \in W, w \neq$ id. In principle, a similar fact does not hold for simple finite-dimensional $\mathfrak{g}$-modules. For instance, there are triangular decompositions such that the corresponding $\rho$ is equal to 0 and so $w .0=0$ for all $w \in W$ even though $\widetilde{V}(0)$ is one dimensional.

However, if $\widetilde{V}(\lambda)$ is finite dimensional and strongly typical, then $\lambda>w \cdot \lambda$ for all $w \in W, w \neq \mathrm{id}$. This can be checked in the following way. Fix a strongly typical 
weight $\nu$ such that $\widetilde{V}(\nu)$ is finite dimensional. Write the character formula (1) in the form

$$
D^{\prime} \operatorname{ch} \widetilde{V}(\nu)=\sum_{w \in W} \operatorname{sn}(w) e^{w \cdot \nu}, \quad \text { where } D^{\prime}:=(\operatorname{ch} \widetilde{M}(0))^{-1} .
$$

Combining the facts that $\operatorname{Stab}_{W}(\nu+\rho)$ is generated by the reflections it contains and that $\operatorname{ch} \widetilde{V}(\nu) \neq 0$, one obtains $w \cdot \nu=w^{\prime} \cdot \nu$ iff $w=w^{\prime}$. A strongly typical Verma module $\widetilde{M}(w . \nu)$ has a finite filtration with the factors of the form $\widetilde{V}\left(w^{\prime} . \nu\right)$ where $w^{\prime} . \nu \leq w \cdot \nu$. Therefore $\operatorname{ch} \widetilde{M}(w \cdot \nu)=\sum_{w^{\prime}} a_{w, w^{\prime}} \operatorname{ch} \widetilde{V}\left(w^{\prime} . \nu\right)$ where $\left(a_{w, w^{\prime}}\right)$ forms "an upper triangular matrix", that is, $a_{w, w^{\prime}}=0$ if $w^{\prime} . \nu \not \leq w \cdot \nu$ and $a_{w, w}=1$. Consequently,

$$
D^{\prime} \operatorname{ch} \widetilde{V}(w \cdot \nu)=\sum_{w^{\prime}} b_{w, w^{\prime}} e^{w^{\prime} \cdot \nu}
$$

where $b_{w, w^{\prime}}=0$ if $w^{\prime} . \nu \nless \leq w . \nu$ and $b_{w, w}=1$. Comparing the last equality with (11), one obtains $w^{\prime} . \nu<\nu$ for all $w^{\prime} \in W, w^{\prime} \neq \mathrm{id}$.

11.2. Lemma. Assume that $N_{1}, N_{2}$ are finite dimensional $\mathfrak{h}$-diagonalizable $\mathfrak{g}$-modules and all simple subquotients of $N_{1} \otimes N_{2}$ are typical. Then $N_{1} \otimes N_{2}$ is a completely reducible module.

Proof. Since central characters separate non-isomorphic typical finite-dimensional modules, it is enough to show that $N_{1} \otimes N_{2}$ does not contain non-trivial extensions $\widetilde{V}$ by $\widetilde{V}$ for any typical finite-dimensional module $\widetilde{V}$. Let $N$ be such an extension. Then the highest weight subspace of $N$ is two dimensional and admits a basis $\left\{v_{1}, v_{2}\right\}$ such that $v_{1}$ is primitive and $v_{1} \in \widetilde{\mathcal{U}} v_{2}$. Then $v_{1} \in \mathcal{U}(\mathfrak{h}) v_{2}$ and so the action of $\mathfrak{h}$ on $N$ is not semisimple. Hence $N_{1} \otimes N_{2}$ does not contain a submodule isomorphic to $N$. The assertion follows.

11.3. Lemma. For any simple finite-dimensional module $\widetilde{V}$ and any $\mu \in \mathfrak{h}^{*}$, the set of $\lambda \in \mathfrak{h}^{*}$ such that $\widetilde{V}(\lambda)$ is finite dimensional and

$$
\operatorname{dim} \operatorname{Hom}_{\mathfrak{g}}\left(\widetilde{V}, \operatorname{Hom}(\widetilde{V}(\lambda-\mu), \widetilde{V}(\lambda))=\left.\operatorname{dim} \widetilde{V}\right|_{\mu}\right.
$$

is Zariski dense in $\mathfrak{h}^{*}$.

Proof. Fix a simple finite-dimensional module $\widetilde{V}$. Let $R$ be the subset of $\mathfrak{h}^{*}$ consisting of the weights $\lambda$ such that $\widetilde{V}(\lambda)$ is a typical finite-dimensional module and the tensor product $\widetilde{V}^{*} \otimes \widetilde{V}(\lambda)$ is the direct sum of typical simple modules. Let us show that $R$ is Zariski dense in $\mathfrak{h}^{*}$.

Indeed, take a finite-dimensional $\mathfrak{g}_{0}$-module $V(\lambda)$. The induced module $\operatorname{Ind}_{\mathfrak{g}_{0}}^{\mathfrak{g}} V(\lambda)$ has a simple submodule $\widetilde{V}\left(\lambda^{\prime}\right)$ which is finite dimensional. The $\mathfrak{g}_{0}$-module $V\left(\lambda^{\prime}\right)$ is a $\mathfrak{g}_{0}$-submodule of $\widetilde{V}\left(\lambda^{\prime}\right)$ and so is a $\mathfrak{g}_{0}$-submodule of $\operatorname{Ind}_{\mathfrak{g}_{0}}^{\mathfrak{g}} V(\lambda)$. As a $\mathfrak{g}_{0}$-module Ind $_{\mathfrak{g}_{0}}^{\mathfrak{g}} V(\lambda) \cong V(\lambda) \otimes \Lambda \mathfrak{g}_{1}$. For finite-dimensional $\mathfrak{g}_{0}$-modules $L, V(\mu)$, the inequality $\operatorname{Hom}_{\mathfrak{g}_{0}}(V(\nu), V(\mu) \otimes L) \neq 0$ implies $(\nu-\mu) \in \Omega(L)$. Therefore $\left(\lambda-\lambda^{\prime}\right) \in \Omega\left(\Lambda \mathfrak{g}_{1}\right)$. Thus for any $\lambda \in \mathfrak{h}^{*}$ such that $V(\lambda)$ is simple, there exists $\lambda^{\prime} \in \lambda+\Omega\left(\Lambda \mathfrak{g}_{1}\right)$ such that $\widetilde{V}\left(\lambda^{\prime}\right)$ is simple. Taking into account that the set of $\lambda^{\prime}$ 's such that $\operatorname{dim} V(\lambda)<\infty$ is Zariski dense in $\mathfrak{h}^{*}$, one concludes that the set of $\lambda^{\prime}$ 's such that $\operatorname{dim} \widetilde{V}(\lambda)<\infty$ is also Zariski dense in $\mathfrak{h}^{*}$.

The condition on weight to be atypical is polynomial (see 2.5.2) and so the set of $\lambda$ 's such that $\widetilde{V}(\lambda)$ is typical finite dimensional is also Zariski dense. A 
module $\widetilde{V}(\lambda)$ is a submodule of $\operatorname{Coind}_{\mathfrak{g}_{0}}^{\mathfrak{g}} V(\lambda)$ and so $\widetilde{V}^{*} \otimes \widetilde{V}(\lambda)$ is a submodule of $\widetilde{V}^{*} \otimes \operatorname{Coind}_{\mathfrak{g}_{0}}^{\mathfrak{g}} V(\lambda)$. Again $\operatorname{Hom}_{\mathfrak{g}_{0}}\left(V(\nu), \widetilde{V}^{*} \otimes \operatorname{Coind}_{\mathfrak{g}_{0}}^{\mathfrak{g}} V(\lambda)\right) \neq 0$ implies $(\nu-\lambda) \in$ $\Omega\left(\widetilde{V}^{*} \otimes \Lambda \mathfrak{g}_{1}\right)$. Therefore if $\widetilde{V}^{*} \otimes \widetilde{V}(\lambda)$ has a subquotient isomorphic to $\widetilde{V}(\nu)$, then $(\nu-\lambda) \in \Omega\left(\widetilde{V}^{*} \otimes \Lambda \mathfrak{g}_{1}\right)$. Using Lemma 11.2 and the fact that the set $\Omega\left(\widetilde{V}^{*} \otimes \Lambda \mathfrak{g}_{1}\right)$ is finite, we conclude that $R$ is Zariski dense in $\mathfrak{h}^{*}$.

Frobenius reciprocity gives

$$
\operatorname{Hom}_{\mathfrak{g}}(\widetilde{V}, \operatorname{Hom}(\widetilde{V}(\lambda-\mu), \widetilde{V}(\lambda))) \cong \operatorname{Hom}_{\mathfrak{g}}\left(\widetilde{V}(\lambda-\mu), \widetilde{V}^{*} \otimes \widetilde{V}(\lambda)\right) .
$$

Take $\lambda \in R$ and denote by $m_{\nu}$ the multiplicity of $\widetilde{V}(\nu)$ in the completely reducible module $\widetilde{V}^{*} \otimes \widetilde{V}(\lambda)$. The character formula (1) gives

$$
D \operatorname{ch}\left(\widetilde{V}^{*} \otimes \widetilde{V}(\lambda)\right)=\sum_{w \in W} \operatorname{sn}(w) e^{w \cdot \lambda} \operatorname{ch} \widetilde{V}^{*}=\sum_{\nu} m_{\nu} \sum_{w \in W} \operatorname{sn}(w) e^{w \cdot \nu} .
$$

For typical finite-dimensional modules $\widetilde{V}(\nu), \widetilde{V}\left(\nu^{\prime}\right)$ the equality $w \cdot \nu=w^{\prime} \cdot \nu^{\prime}$ implies $\nu=\nu^{\prime}$ and $w=w^{\prime}$ (see 11.1). Therefore $\operatorname{dim} \operatorname{Hom}_{\mathfrak{g}}\left(\widetilde{V}(\lambda-\mu), \widetilde{V}(\lambda) \otimes \widetilde{V}^{*}\right)=m_{\lambda-\mu}$ is equal to the coefficient of the term $e^{\lambda-\mu}$ in the expression $\sum_{w \in W} \operatorname{sn}(w) e^{w \cdot \lambda} \operatorname{ch} \widetilde{V}^{*}$. For "sufficiently large" $\lambda \in R$ this coefficient is equal to $\left.\operatorname{dim} \widetilde{V}^{*}\right|_{-\mu}$. More precisely, take $\lambda \in R, w \neq$ id and $\alpha \in \pi_{0}$ such that $s_{\alpha} w<w$. Then $w^{-1} \alpha \in \Delta_{0}^{-}$and so $(w(\lambda+\rho), \alpha)=\left(\lambda+\rho, w^{-1} \alpha\right)<0$ by 11.1 Then

$$
(\lambda-\mu-w \cdot \lambda, \alpha)=(\lambda+\rho-\mu, \alpha)-(w(\lambda+\rho), \alpha)>(\lambda+\rho-\mu, \alpha) .
$$

As a consequence, for any $\lambda$ belonging to the set

$$
R_{1}:=\left\{\lambda \in R \mid(\lambda, \alpha)>(\xi+\mu-\rho, \alpha) \quad \forall \xi \in \Omega\left(\widetilde{V}^{*}\right), \alpha \in \pi_{0}\right\}
$$

the inclusion $(\lambda-\mu) \in\left(w \cdot \lambda+\Omega\left(\widetilde{V}^{*}\right)\right)$ implies $w=$ id. Hence $m_{\lambda-\mu}=\left.\operatorname{dim} \widetilde{V}^{*}\right|_{-\mu}=$ $\left.\operatorname{dim} \widetilde{V}\right|_{\mu}$ for any $\lambda \in R_{1}$.

For any $\lambda \in R$ and $\alpha \in \pi_{0}$, the value $(\lambda, \alpha)$ belongs to $\mathbb{N}^{+}$, since the $\mathfrak{g}_{0}$-module $V(\lambda)$ is finite dimensional. Thus $R_{1}$ is obtained from $R$ by removing the points lying at finitely many hyperplanes. Taking into account that $R$ is Zariski dense in $\mathfrak{h}^{*}$, one concludes that $R_{1}$ is also Zariski dense. This completes the proof.

11.4. Lemma. For all $\lambda, \nu \in \mathfrak{h}^{*}$ and all simple finite-dimensional $\widetilde{V}$ one has

$$
\operatorname{dim} \operatorname{Hom}_{\mathfrak{g}}\left(\widetilde{V}, \operatorname{Hom}\left(\widetilde{M}(\lambda-\mu), \widetilde{M}(\lambda)^{\#}\right)\right)=\left.\operatorname{dim} \widetilde{V}\right|_{\mu} .
$$

Proof. Frobenius reciprocity gives

$$
\operatorname{Hom}_{\mathfrak{g}}\left(\widetilde{V}, \operatorname{Hom}\left(\widetilde{M}(\lambda-\mu), \widetilde{M}(\lambda)^{\#}\right)\right) \cong \operatorname{Hom}_{\mathfrak{g}}\left(\widetilde{M}(\lambda-\mu), \widetilde{M}(\lambda)^{\#} \otimes \widetilde{V}^{*}\right) .
$$

Using the notation of 2.5.1 one has

$$
\operatorname{Hom}_{\mathfrak{g}}\left(\widetilde{M}(\lambda-\mu), \widetilde{M}(\lambda)^{\#} \otimes \widetilde{V}^{*}\right) \cong \operatorname{Hom}_{\mathfrak{b}}\left(\mathbb{C}_{\lambda-\mu}, \widetilde{M}(\lambda)^{\#} \otimes \widetilde{V}^{*}\right) .
$$

All Verma modules are isomorphic as $\mathfrak{n}^{-}$-modules. Therefore their duals are isomorphic as $\mathfrak{n}^{+}$-modules. Furthermore for any $\lambda^{\prime} \in \mathfrak{h}^{*}$ the $\mathfrak{b}$-modules $\widetilde{M}(\lambda)^{\#}$ and $\widetilde{M}\left(\lambda^{\prime}\right)^{\#} \otimes \mathbb{C}_{\lambda-\lambda^{\prime}}$ are isomorphic. Taking into account (12), one concludes that

$$
k:=\operatorname{dim} \operatorname{Hom}_{\mathfrak{g}}\left(\widetilde{V}, \operatorname{Hom}\left(\widetilde{M}(\lambda-\mu), \widetilde{M}(\lambda)^{\#}\right)\right)
$$

does not depend on $\lambda$ (for fixed $\widetilde{V}$ and $\mu$ ). 
Recall that $\widetilde{V}(\lambda-\mu)$ is a quotient of $\widetilde{M}(\lambda-\mu)$ and $\widetilde{V}(\lambda)$ is a submodule of $\widetilde{M}(\lambda) \#$. Consequently $k \geq \operatorname{dim}_{\operatorname{Hom}}\left(\widetilde{V}(\lambda-\mu), \widetilde{V}(\lambda) \otimes \widetilde{V}^{*}\right)$ for all $\lambda \in \mathfrak{h}^{*}$. Using Lemma 11.3 one obtains $k \geq\left.\operatorname{dim} \widetilde{V}\right|_{\mu}$.

To verify that $k \leq\left.\operatorname{dim} \widetilde{V}\right|_{\mu}$ fix $\lambda$ such that $\widetilde{M}(\lambda)^{\#}$ is simple. Denote by $m_{1}$ (resp., $m_{2}$ ) a highest weight vector of $\widetilde{M}(\lambda-\mu)$ (resp., $\left.\widetilde{M}(\lambda)^{\#}\right)$. Consider a map

$$
\begin{aligned}
& \iota:\left.\operatorname{Hom}_{\mathfrak{g}}\left(\widetilde{V}, \operatorname{Hom}\left(\widetilde{M}(\lambda-\mu), \widetilde{M}(\lambda)^{\#}\right)\right) \rightarrow \widetilde{V}^{*}\right|_{-\mu} \text { s.t } \\
& \iota(\psi)(v) m_{2}=\left.\psi(v)\left(m_{1}\right) \quad \forall v \in \widetilde{V}\right|_{\mu}, \psi \in \operatorname{Hom}_{\mathfrak{g}}\left(\widetilde{V}, \operatorname{Hom}\left(\widetilde{M}(\lambda-\mu), \widetilde{M}(\lambda)^{\#}\right)\right) .
\end{aligned}
$$

Let us show that $\iota$ is a monomorphism. Take a non-zero element

$$
\psi \in \operatorname{Hom}_{\mathfrak{g}}\left(\widetilde{V}, \operatorname{Hom}\left(\widetilde{M}(\lambda-\mu), \widetilde{M}(\lambda)^{\#}\right)\right) .
$$

The vector space $\psi(\widetilde{V})(\widetilde{M}(\lambda-\mu))$ is a non-zero $\mathfrak{g}$-submodule of a simple module $\widetilde{M}(\lambda)^{\#}$; thus it coincides with $\widetilde{M}(\lambda)^{\#}$. One has

$$
\psi(\widetilde{V})\left(M_{1}\right)=\psi(\widetilde{V})\left(\mathcal{U}\left(\mathfrak{n}^{-}\right) m_{1}\right)=\mathcal{U}\left(\mathfrak{n}^{-}\right) \psi(\widetilde{V})\left(m_{1}\right)
$$

since $\psi(\widetilde{V})$ is ad $\mathfrak{g}$-stable. Therefore $\psi(\widetilde{V})\left(m_{1}\right)$ contains the highest weight vector $m_{2}$, that is, $\psi(v)\left(m_{1}\right)=m_{2}$ for a certain $v \in \widetilde{V}$. Obviously one can choose $v$ to be a weight vector; then $\left.v \in \widetilde{V}\right|_{\mu}$ and $\iota(\psi)(v)=1$. Hence $\iota$ is a monomorphism and so $k \leq\left.\operatorname{dim} \widetilde{V}^{*}\right|_{-\mu}=\left.\operatorname{dim} \widetilde{V}\right|_{\mu}$ as required.

11.4.1. Remark. If $\widetilde{M}(\lambda)$ is simple, the above lemma gives

$$
\operatorname{dim} \operatorname{Hom}_{\mathfrak{g}}(\widetilde{V}, \operatorname{Hom}(\widetilde{M}(\lambda), \widetilde{M}(\lambda)))=\left.\operatorname{dim} \widetilde{V}\right|_{0}
$$

for any simple finite-dimensional $\widetilde{V}$.

11.5. Lemma. Let $A$ be a polynomial algebra, and let $W$ be a finite group acting on A. Assume that $p, p^{\prime} \in A$ are such that $p^{\prime} / p$ is $W$-invariant and let $q$ be a maximal $W$-invariant divisor of $p^{|W|}$. Then there exist $q^{\prime} \in A^{W}$ such that $p^{\prime} / p=q^{\prime} / q$.

Proof. Any non-zero polynomial has a unique factorization into irreducible ones. Let $a / b$ be a reduced form of the fraction $p^{\prime} / p$. For any $s \in W$ one has $s(a) / s(b)=$ $a / b$ and so $b / s(b)$ is a scalar. Since $W$ is finite, $1=(b / s(b))^{|W|}=b^{|W|} / s\left(b^{|W|}\right)$. Hence $b^{|W|} \in A^{W}$. Since $p$ is divisible by $b, p^{|W|}$ is divisible by $b^{|W|}$. Therefore $q$ is divisible by $b^{|W|}$. Then there exist $q^{\prime} \in A$ such that $q^{\prime} / q=a / b=p^{\prime} / p$. The $W$-invariance of both $q$ and $p^{\prime} / p$ implies the $W$-invariance of $q^{\prime}$.

\section{ACKNOWLEDGMENTS}

It is a pleasant duty to express gratitude to my teacher A. Joseph. Many ideas in this paper are owed to his book and to his courses given at the Weizmann Institute. The author would like to thank M. Duflo, E. Lanzmann, I. Musson and I. Penkov for helpful discussions and V. Serganova for her extreme patience and useful comments. This work was done when the author was a postdoctoral fellow at MSRI. The author would like to thank MSRI and the organizers of the Noncommutative Algebra Program for their support and hospitality. 


\section{REFERENCES}

[AL] M. Aubry and J.-M. Lemaire, Zero divisors in enveloping algebras of graded Lie algebras, J. Pure Appl. Algebra 38 (1985), 159-166. MR 87a:17022

[BF] A. D. Bell, R. Farnsteiner, On the theory of Frobenius extensions and its application to Lie superalgebras, Trans. AMS 335 (1993), no.1, 407-424. MR 93c:17049

[BL] J. Bernstein, V. Lunts, A simple proof of Kostant's theorem that $\widetilde{\mathcal{U}}$ is free over its center, Amer. J. Math. 118 (1996), no.5, 979-987. MR 97h:17012

[BZV] M. Bershadsky, S. Zhukov, A. Vaintrob, $\mathfrak{p s l}(n \mid n)$ sigma model as a conformal field theory, Nuclear Phys. B 559 (1999), no.1-2, 205-234. MR 2001b:81128

[D] M. Duflo, Construction of primitive ideals in an enveloping algebra, in: I. M. Gelfand, ed., Publ. of 1971 Summer School in Math., Janos Bolyai Math. Soc., Budapest, 77-93. MR 53:3045

[FSS] L. Frappat, P. Sorba, A. Sciarrino, Dictionary on Lie superalgebras, hep-th/9607161.

[G1] M. Gorelik, On the ghost centre of Lie superalgebras, Ann. Inst. Fourier 50 (2000), no.6, 1745-1764.

[G2] M. Gorelik, Strongly typical representations of the basic classical Lie superalgebras, preprint (2000).

[GL1] M. Gorelik, E. Lanzmann, The annihilation theorem for the completely reducible Lie superalgebras, Invent. Math. 137 (1999), 651-680. MR 2000i:17017

[GL2] M. Gorelik, E. Lanzmann, The minimal primitive spectrum of the enveloping algebra of the Lie superalgebra osp $(1,2 l)$, Adv. in Math. 154 (2000), no.2, 333-366. MR 2001g:17023

[Ja] J. C. Jantzen, "Einhüllende Algebren Halbeinfacher Lie-Algebren", Springer-Verlag (1983). MR 86c:17011

[J1] A. Joseph, Kostant's problem, Goldie rank and the Gelfand-Kirillov conjecture, Invent. Math. 56 (1980), 193-204. MR 82f:17008

[J2] A. Joseph, Quantum groups and their primitive ideals, Springer-Verlag (1995). MR 96d:17015

[J3] A. Joseph, Sur l'annulateur d'un module de Verma, NATO Adv. Sci. Inst. Ser. C Math. Phys. Sci. 514 (1998). MR 99k:17022

[JL] A. Joseph, G. Letzter, Verma modules annihilators and quantized enveloping algebras, Ann. Ec. Norm. Sup. série 4, t. 28 (1995), 493-526. MR 96i:17011

[K1] V. G. Kac, Lie superalgebras, Adv. in Math. 26 (1977), 8-96. MR 58:5803

[K2] V. G. Kac, Characters of typical representations of Lie superalgebras, Comm. Alg. 5 (1977), 889-997. MR 56:3075

[K3] V. G. Kac, Representations of classical Lie superalgebras, Lecture Notes in Math., SpringerVerlag, Berlin, 676 (1978), 597-626. MR 80f:17006

[Ko] B. Kostant, Lie group representations on polynomial rings, Amer. J. Math. 85 (1963), 327-404. MR 28:1252

[LM] E. S. Letzter, I. M. Musson, Complete sets of representations of classical Lie superalgebras, Lett. Math. Phys. 31 (1994), 247-253. MR 95g:17008

[McL] S. Mac Lane, Homology, Springer-Verlag (1963). MR 28:122

[M1] I. M. Musson, On the center of the enveloping algebra of a classical simple Lie superalgebra, J. of Algebra 193 (1997), 75-101. MR 98k:17012

[M2] I. M. Musson, A classification of primitive ideals in the enveloping algebra of a classical simple Lie superalgebra, Adv. in Math. 91 (1992), 252-268. MR 93c:17022

[PRV] K. P. Parthasarathy, R. Ranga-Rao, V. S. Varadarajan, Representations of complex semisimple Lie groups and Lie algebras, Ann. Math. 85 (1967), 383-429. MR 37:1526

[PS1] I. Penkov, V. Serganova, Representation of classical Lie superalgebras of type I, Indag. Mathem., N.S. 3 (4) (1992), 419-466. MR 93k:17006

[PS2] I. Penkov, V. Serganova, Generic irreducible representations of finite-dimensional Lie superalgebras, International J. Math., Vol. 5, No. 3 (1994), 389-419. MR 95c:17015

[Sch] M. Scheunert, The theory of Lie superalgebras, Lect. Notes in Math., 716 Springer-Verlag (1979). MR 80i:17005

[S1] A. N. Sergeev, Invariant polynomial functions on Lie superalgebras, C.R. Acad. Bulgare Sci. 35 (1982), no.5, 573-576. MR 84h:17015

Department of Mathematics, Weizmann Institute of Science, Rehovot 76100, Israel

E-mail address: gorelik@wisdom.weizmann.ac.il 